Supporting Information:

\title{
The Mechanism of the Self-Initiated Thermal Polymerization of Styrene. Theoretical Solution of a Classic Problem
}

\author{
Kelli S. Khuong, Walter H. Jones, William A. Pryor, and K. N. Houk* \\ Department of Chemistry and Biochemistry, University of California, Los Angeles, CA 90095- \\ 1569, and Department of Chemistry, Louisiana State University, Baton Rouge, CA 70803-1804
}

Contents:

Table 1: Energies of all stationary points relevant to the study of styrene self-initiation. Calculated energies include electronic energies at $0 \mathrm{~K}$, enthalpies at $0 \mathrm{~K}$, enthalpies at $298 \mathrm{~K}$, free energies at $298 \mathrm{~K}$, and entropies at $298 \mathrm{~K}$. Optimizations were done using B3LYP/6-31G(d) or BPW91/6-31G(d). R and U refer to restricted and unrestricted. Where appropriate, triplet single point energies, imaginary frequencies and $\left\langle\mathrm{S}^{2}\right\rangle$ values are included.

$B 3 L Y P$

BPW91

Table 2: B3LYP and BPW91 Cartesian coordinates for all stationary points listed in Table 1.

B3LYP

BPW91

Table 3: Raw data from Figures 12 and 13. B3LYP/6-31G(d) and BPW91/6-31G(d) constrained optimizations of two molecules of styrene in the gauche/sickle conformation. Bonds lengths $\mathrm{A} / \mathrm{B}$ or $\mathrm{A} / \mathrm{C}$ were constrained. S27-S30 
Table 1. Energies of all stationary points relevant to the study of styrene self-initiation. Calculated energies include electronic energies at $0 \mathrm{~K}$, enthalpies at $0 \mathrm{~K}$, enthalpies at $298 \mathrm{~K}$, free energies at $298 \mathrm{~K}$, and entropies at $298 \mathrm{~K}$. Optimizations were done using B3LYP/6-31G(d) or BPW91/6-31G(d). R and $U$ refer to restricted and unrestricted. Where appropriate, triplet single point energies, imaginary frequencies and $<\mathrm{S}^{2}>$ values are included.

\begin{tabular}{|c|c|c|c|c|c|c|c|c|c|c|}
\hline Entry & & & E, OK (au) & H, 0K (au) & H, 298K (au) & G, 298K (au) & $\begin{array}{c}\text { S, 298K } \\
\text { (eu) }\end{array}$ & $\begin{array}{l}\text { E, triplet, } \\
\text { 0K (au) }\end{array}$ & $\begin{array}{c}\text { Imag } \\
\text { freq } \\
\left(\mathrm{cm}^{-1}\right)\end{array}$ & $\left\langle\mathrm{S}^{2}\right\rangle$ \\
\hline \multicolumn{11}{|c|}{ B3LYP/6-31G(d): } \\
\hline $1 \mathrm{a}$ & Styrene monomer & $\mathrm{R}$ & -309.648259 & -309.514526 & -309.506811 & -309.546011 & 82.505 & & & \\
\hline $2 \mathrm{a}$ & AHx-TSDA & $\mathrm{R}$ & -619.253747 & -618.983303 & -618.969438 & -619.023277 & 113.314 & & -122 & \\
\hline 3a & AHn-TSDA & $\mathrm{R}$ & -619.251192 & -618.980998 & -618.967062 & -619.021426 & 114.419 & & -136 & \\
\hline $4 a$ & BHx-TSDA & $\mathrm{R}$ & -619.241813 & -618.971790 & -618.957748 & -619.012023 & 114.231 & & -606 & \\
\hline $5 a$ & BHn-TSDA & $\mathrm{R}$ & -619.241111 & -618.970894 & -618.956921 & -619.010872 & 113.550 & & -603 & \\
\hline $6 a$ & TS1 (At) & $\mathrm{U}$ & -619.256344 & -618.987923 & -618.973141 & -619.031275 & 122.354 & -619.235068 & -804 & 0.44 \\
\hline $7 a$ & TS1 (Ac) & $\mathrm{U}$ & -619.256768 & -618.988127 & -618.973443 & -619.030972 & 121.080 & -619.235206 & -792 & 0.42 \\
\hline $8 a$ & TS1 (Gs) & $\mathrm{U}$ & -619.256742 & -618.987965 & -618.973389 & -619.030102 & 119.363 & -619.233424 & -772 & 0.35 \\
\hline $9 a$ & TS1 (Gu) & $\mathrm{U}$ & -619.254792 & -618.986388 & -618.971710 & -619.029020 & 120.620 & -619.232820 & -749 & 0.37 \\
\hline $10 \mathrm{a}$ & TS1 (Gw) & $\mathrm{U}$ & -619.252938 & -618.984909 & -618.970049 & -619.028544 & 123.297 & -619.233560 & -804 & 0.49 \\
\hline $11 \mathrm{a}$ & •M2•(At) & $\mathrm{U}$ & -619.264394 & -618.995150 & -618.980136 & -619.039011 & 123.914 & -619.261939 & & 0.99 \\
\hline $12 \mathrm{a}$ & •M2•(Ac) & $\mathrm{U}$ & -619.264481 & -618.995123 & -618.980185 & -619.038190 & 122.081 & -619.262031 & & 0.99 \\
\hline $13 a$ & •M2•(Gs) & $\mathrm{U}$ & -619.263712 & -618.994412 & -618.979468 & -619.037790 & 122.750 & -619.261055 & & 0.99 \\
\hline $14 a$ & $\bullet \mathrm{M} 2 \bullet(\mathrm{Gu})$ & $\mathrm{U}$ & -619.262651 & -618.993658 & -618.978638 & -619.037221 & 123.297 & -619.260008 & & 0.99 \\
\hline $15 a$ & $\bullet \mathrm{M} 2 \bullet(\mathrm{Gw})$ & $\mathrm{U}$ & -619.262670 & -618.993589 & -618.978533 & -619.038458 & 126.124 & -619.261790 & & 1.04 \\
\hline $16 a$ & TS2x & $\mathrm{U}$ & -619.255612 & -618.985312 & -618.971607 & -619.025069 & 112.521 & -619.230994 & -342 & 0.33 \\
\hline $17 \mathrm{a}$ & TS2n & $\mathrm{U}$ & -619.253485 & -618.983381 & -618.969638 & -619.023690 & 113.763 & -619.230240 & -327 & 0.37 \\
\hline $18 \mathrm{a}$ & TScisDCB & $\mathrm{U}$ & -619.258701 & -618.989649 & -618.975361 & -619.031541 & 118.242 & -619.247651 & -242 & 0.75 \\
\hline 19a & TStransDCB & $\mathrm{U}$ & -619.261533 & -618.992373 & -618.978127 & -619.034246 & 118.113 & -619.249938 & -182 & 0.74 \\
\hline $20 a$ & TStransDCB' & $\mathrm{U}$ & -619.256965 & -618.988078 & -618.973702 & -619.029908 & 118.294 & -619.247643 & -294 & 0.80 \\
\hline $21 \mathrm{a}$ & $\mathrm{AHx}$ & $\mathrm{R}$ & -619.301928 & -619.027683 & -619.014079 & -619.067359 & 112.136 & & & \\
\hline $22 \mathrm{a}$ & AHn & $\mathrm{R}$ & -619.298360 & -619.023900 & -619.010409 & -619.063462 & 111.658 & & & \\
\hline $23 a$ & $\mathrm{BHx}$ & $\mathrm{R}$ & -619.300864 & -619.026291 & -619.012874 & -619.065716 & 111.215 & & & \\
\hline $24 a$ & $\mathrm{BHn}$ & $\mathrm{R}$ & -619.302418 & -619.028264 & -619.014646 & -619.068193 & 112.699 & & & \\
\hline $25 a$ & cis-DCB & $\mathrm{R}$ & -619.313525 & -619.039277 & -619.025486 & -619.080401 & 115.578 & & & \\
\hline $26 a$ & trans-DCB & $\mathrm{R}$ & -619.319381 & -619.045279 & -619.031391 & -619.086133 & 115.214 & & & \\
\hline $27 a$ & AHx + M: TS Habs & $\mathrm{U}$ & -928.907639 & -928.503642 & -928.482302 & -928.554700 & 152.376 & -928.876343 & -2040 & 0.04 \\
\hline $28 a$ & AHn + M: TS Habs & $\mathrm{U}$ & -928.908916 & -928.505222 & -928.483786 & -928.557240 & 154.597 & -928.880010 & -2076 & 0.11 \\
\hline $29 a$ & AHx + M: TS Ene1 & $\mathrm{R}$ & -928.915441 & -928.510029 & -928.489185 & -928.559571 & 148.141 & & -1329 & \\
\hline $30 \mathrm{a}$ & AHn + M: TS Ene1 & $\mathrm{R}$ & -928.916148 & -928.510683 & -928.489886 & -928.559784 & 147.114 & & -1293 & \\
\hline $31 a$ & AHn + M: TS Ene2 & $\mathrm{R}$ & -928.914006 & -928.508789 & -928.487833 & -928.558490 & 148.709 & & -1330 & \\
\hline
\end{tabular}


AHn + M: TS Ene3, $\beta-$ regio

A-

phenylethyl rad (HM•)

•M2• + M: TS Habs

M2•

$\mathrm{HM} \bullet+\mathrm{M}$ : TS mono

•M2• + M: TS dirad

$2 \mathrm{HM} \cdot \mathrm{TS}$ disp1

2 HM• TS disp2

AHn + HM•: TS tr1a

AHx + HM•: TS tr1a

cis A-Sty (from AHx)

trans A-Sty (from AHn)

LYP Model systems, AH intermediate

\begin{tabular}{|c|c|c|c|c|}
\hline $45 a$ & AH model & $\mathrm{R}$ & -388.252638 & -388.059421 \\
\hline $46 a$ & $\begin{array}{l}\text { AH model + M: TS Habs1a } \\
\text { AH model + M: TS }\end{array}$ & $\mathrm{U}$ & -697.861303 & -697.538885 \\
\hline $47 a$ & Habs1b & $\mathrm{U}$ & -697.862076 & -697.539225 \\
\hline $48 \mathrm{a}$ & AH model + M: TS Ene1 & $\mathrm{R}$ & -697.868514 & -697.544179 \\
\hline $49 a$ & AH model + M: TS Ene2 & $\mathrm{R}$ & -697.866594 & -697.542395 \\
\hline $50 a$ & AH model + M: TS Ene3 & $\mathrm{R}$ & -697.866744 & -697.539126 \\
\hline $51 \mathrm{a}$ & AH model + HM•: TS tr1a & $\mathrm{U}$ & -698.472644 & -698.139090 \\
\hline $52 \mathrm{a}$ & AH model + HM•: TS tr1b & $\mathrm{U}$ & -698.472370 & -698.138888 \\
\hline $53 a$ & AH model + HM•: TS tr2a & $\mathrm{U}$ & -698.472640 & -698.139095 \\
\hline $54 a$ & AH model + HM•: TS tr2b & $\mathrm{U}$ & -698.471565 & -698.138068 \\
\hline $55 a$ & AH model + HM•: TS tr3a & $\mathrm{U}$ & -698.472266 & -698.138776 \\
\hline $56 a$ & AH model $+\mathrm{HM} \bullet:$ TS tr3b & $\mathrm{U}$ & -698.471565 & -698.138068 \\
\hline
\end{tabular}

56a AH model + HM॰: TS tr3b U

\section{$-928.913745$ \\ $-928.505092$}

$-618.711080$

$-618.449388$

$-310.233367$

$-928.874695$

$-618.683877$

$-619.870739$

$-928.901667$

$-620.461058$

$-620.459419$

$-929.519456$

$-929.517796$

$-929.025747$

$-310.090134$

$-928.475718$

$-618.424999$

$-619.591858$

$-928.497187$

$-620.174971$

$-620.173857$

$-929.104653$

$-929.103135$

$-928.611802$

$-928.611802$

$-928.484711$

$-618.435994$

$-310.081755$

$-928.452768$

$-618.410211$

$-619.576289$

$-928.474680$

$-620.158951$

$-620.157595$

$-929.082641$

$-929.081190$

$-928.591216$

$-928.589565$

styrene and ethyle

$\begin{array}{lll}-388.050449 & -388.092001 & 87.452\end{array}$

$\begin{array}{lll}-697.522040 & -697.584086 & 130.587\end{array}$

$-697.522499$

$-697.528040$

$-697.526135$

$-697.523323$

$-698.121680$

$-698.121438$

$-698.121679$

$-698.120641$

$-698.121363$

$-698.120641$
$-928.554521 \quad 146.928$

112.491

$-310.122738 \quad 86.256$

$-928.533343 \quad 169.584$

$-618.469753 \quad 125.318$

$-619.636705 \quad 127.154$

$-928.553279 \quad 165.426$

$-620.220116 \quad 128.733$

$-620.220080 \quad 131.510$

-929.157573 157.707

-929.154719 154.755

-928.661599 148.132

$-928.659503 \quad 147.198$

$-697.584049 \quad 129.542$

$-697.586410 \quad 122.850$

$-697.585109 \quad 124.121$

$-697.581859123 .199$

$-698.185546 \quad 134.418$

$-698.185393-134.605$

$-698.185857 \quad 135.074$

$-698.184032 \quad 133.418$

$-698.185453 \quad 134.888$

$-698.184035 \quad 133.425$
$-476$

0.78

0.78

$\begin{array}{lll}-928.874230 & -1991 & 1.07\end{array}$

0.78

$\begin{array}{ll}-448 & 0.79\end{array}$

$\begin{array}{lll}-928.899884 & -432 & 1.02\end{array}$

$\begin{array}{lll}-620.439647 & -836 & 0.46\end{array}$

$\begin{array}{lll}-620.442355 & -1033 & 0.58\end{array}$

$-1459 \quad 0.80$

$-1567 \quad 0.81$ 


\begin{tabular}{|c|c|c|c|c|c|c|c|c|c|c|}
\hline Entry & & & E, OK (au) & H, 0K (au) & H, 298K (au) & G, 298K (au) & $\begin{array}{l}\text { S, 298K } \\
\quad \text { (eu) }\end{array}$ & $\begin{array}{l}\text { E, triplet, } \\
\text { OK (au) }\end{array}$ & $\begin{array}{c}\text { Imag } \\
\text { freq } \\
\left(\mathrm{cm}^{-1}\right)\end{array}$ & $\left\langle\mathrm{S}^{2}\right\rangle$ \\
\hline \multicolumn{11}{|c|}{ BPW91/6-31G(d): } \\
\hline $1 b$ & Styrene monomer & $\mathrm{R}$ & -309.600601 & -309.470322 & -309.462419 & -309.501881 & 83.055 & & & \\
\hline $4 \mathrm{~b}$ & BHx-TSDA & $\mathrm{R}$ & -619.156725 & -618.893870 & -618.879305 & -618.934712 & 116.614 & & -488 & \\
\hline $5 b$ & BHn-TSDA & $\mathrm{R}$ & -619.210409 & -618.943517 & -618.929495 & -618.983787 & 114.266 & & -498 & \\
\hline $6 b$ & $\mathrm{TS} 1$ (At) & $\mathrm{U}$ & -619.170407 & -618.908333 & -618.893216 & -618.952034 & 123.795 & & -619 & 0.19 \\
\hline $7 b$ & TS1 (Ac) & $\mathrm{U}$ & -619.170608 & -618.908502 & -618.893455 & -618.951729 & 122.647 & & -573 & 0.18 \\
\hline $8 b$ & TS1(Gs) & $\mathrm{U}$ & -619.170926 & -618.908538 & -618.893663 & -618.950775 & 120.204 & & -407 & 0.09 \\
\hline $9 b$ & $\mathrm{TS} 1(\mathrm{Gu})$ & $\mathrm{U}$ & -619.168115 & -618.906037 & -618.891047 & -618.948849 & 121.653 & & -571 & 0.12 \\
\hline $10 \mathrm{~b}$ & TS1(Gw) & $\mathrm{U}$ & -619.166575 & -618.904894 & -618.889700 & -618.948817 & 124.423 & & -640 & 0.25 \\
\hline $11 b$ & •M2•(At) & $\mathrm{U}$ & -619.171867 & -618.909213 & -618.893809 & -618.953398 & 125.415 & & & 0.78 \\
\hline $12 b$ & •M2•(Ac) & $\mathrm{U}$ & -619.171737 & -618.908996 & -618.893672 & -618.952296 & 123.385 & & & 0.78 \\
\hline $13 \mathrm{~b}$ & •M2•(Gs) & $\mathrm{U}$ & -619.171268 & -618.908394 & -618.893134 & -618.951315 & 122.453 & & & 0.61 \\
\hline $14 \mathrm{~b}$ & •M2•(Gu) & $\mathrm{U}$ & -619.169685 & -618.907134 & -618.891777 & -618.950707 & 124.029 & & & 0.77 \\
\hline $15 b$ & •M2•(Gw) & $\mathrm{U}$ & -619.169249 & -618.906816 & -618.891350 & -618.952349 & 128.383 & & & 0.92 \\
\hline $16 b$ & TS2x & $\mathrm{U}$ & -619.170639 & -618.906733 & -618.892634 & -618.946763 & 113.924 & & -60 & 0.00 \\
\hline $17 \mathrm{~b}$ & $\operatorname{TS} 2 \mathrm{n}$ & $\mathrm{U}$ & -619.167324 & -618.904520 & -618.890103 & -618.945836 & 117.299 & & -114 & 0.07 \\
\hline $18 b$ & TScisDCB & $\mathrm{U}$ & -619.167028 & -618.904626 & -618.890040 & -618.946233 & 118.267 & & -91 & 0.76 \\
\hline $19 b$ & TStransDCB & $\mathrm{U}$ & -619.169948 & -618.907849 & -618.893254 & -618.951453 & 122.490 & & -23 & 0.81 \\
\hline $20 b$ & TStransDCB' & $\mathrm{U}$ & -619.164604 & -618.902530 & -618.887741 & -618.944703 & 119.886 & & -186 & 0.85 \\
\hline $21 b$ & $\mathrm{AHx}$ & $\mathrm{R}$ & -619.209896 & -618.942919 & -618.928910 & -618.982940 & 113.717 & & & \\
\hline $22 b$ & $\mathrm{AHn}$ & $\mathrm{R}$ & -619.206466 & -618.939209 & -618.925337 & -618.978906 & 112.744 & & & \\
\hline $23 b$ & $\mathrm{BHx}$ & $\mathrm{R}$ & -619.209161 & -618.941757 & -618.927967 & -618.981372 & 112.399 & & & \\
\hline $24 b$ & $\mathrm{BHn}$ & $\mathrm{R}$ & -619.210409 & -618.943517 & -618.929495 & -618.983787 & 114.266 & & & \\
\hline $25 b$ & cis-DCB & $\mathrm{R}$ & -619.221534 & -618.954343 & -618.940154 & -618.995756 & 117.024 & & & \\
\hline $26 b$ & trans-DCB & $\mathrm{R}$ & -619.227768 & -618.960707 & -618.946409 & -619.002017 & 117.037 & & & \\
\hline $27 b$ & AHx + M: TS Habs & $\mathrm{U}$ & -928.779097 & -928.385200 & -928.363298 & -928.436532 & 154.135 & & -1021 & 0.00 \\
\hline $28 b$ & AHn + M: TS Habs & $\mathrm{U}$ & -928.780903 & -928.387166 & -928.365183 & -928.439285 & 155.960 & & -980 & 0.00 \\
\hline $29 b$ & AHx + M: TS Ene1 & $\mathrm{R}$ & -928.785016 & -928.390228 & -928.368696 & -928.440410 & 150.934 & & -1038 & \\
\hline $30 b$ & AHn + M: TS Ene1 & $\mathrm{R}$ & -928.785775 & -928.390910 & -928.369423 & -928.440775 & 150.174 & & -998 & \\
\hline $33 b$ & $A \bullet$ & $\mathrm{U}$ & -618.620942 & -618.365866 & -618.352075 & -618.406279 & 114.081 & & & 0.77 \\
\hline $34 b$ & phenylethyl rad (HM•) & $\mathrm{U}$ & -310.184106 & -310.044297 & -310.035732 & -310.077058 & 86.977 & & & 0.77 \\
\hline $35 b$ & $\cdot \mathrm{M} 2 \bullet+\mathrm{M}$ : TS Habs & $\mathrm{U}$ & -928.743694 & -928.354541 & -928.331027 & -928.412506 & 171.488 & & -1533 & 0.95 \\
\hline $36 b$ & $\mathrm{M} 2 \bullet$ & $\mathrm{U}$ & -618.592319 & -618.340013 & -618.324826 & -618.385463 & 127.623 & & & 0.76 \\
\hline $37 \mathrm{~b}$ & HM• + M: TS mono & $\mathrm{U}$ & -619.776418 & -619.504572 & -619.488541 & -619.549821 & 128.973 & & -317 & 0.77 \\
\hline $38 b$ & •M2•+ M: TS dirad & $\mathrm{U}$ & -928.763732 & -928.369272 & -928.346166 & -928.426007 & 168.040 & & -306 & 0.83 \\
\hline $43 b$ & cis A-Sty (from AHx) & $\mathrm{R}$ & -928.884151 & -928.480804 & -928.459632 & -928.530941 & 150.082 & & & \\
\hline $44 \mathrm{~b}$ & trans A-Sty (from AHn) & $\mathrm{R}$ & -928.882694 & -928.479125 & -928.458018 & -928.528951 & 149.292 & & & \\
\hline
\end{tabular}


Table 2. B3LYP and BPW91 Cartesian coordinates for all stationary points listed in Table 1.

1a) Styrene monomer RB3LYP/6-31G*

$\begin{array}{rrrr}6 & 1.362413 & 1.329543 & 0.000003 \\ 6 & -0.008765 & 1.092354 & 0.000003 \\ 6 & -0.515244 & -0.220369 & 0.000014 \\ 6 & 0.406461 & -1.281392 & -0.000007 \\ 6 & 1.780745 & -1.046231 & -0.000006 \\ 6 & 2.265361 & 0.261832 & 0.000002 \\ 1 & 1.730459 & 2.352333 & -0.000002 \\ 1 & -0.693773 & 1.935336 & 0.000015 \\ 1 & 0.034866 & -2.303845 & -0.000010 \\ 1 & 2.471841 & -1.885095 & -0.000002 \\ 1 & 3.335516 & 0.450417 & -0.000021 \\ 6 & -1.954800 & -0.529184 & -0.000001 \\ 1 & -2.186197 & -1.594643 & 0.000070 \\ 6 & -2.977460 & 0.335020 & -0.000014 \\ 1 & -4.004472 & -0.016996 & 0.000004 \\ 1 & -2.840507 & 1.413059 & -0.000020\end{array}$

2a) $\underline{A H x-T S D A}$

RB3LYP/6-31G*

$\begin{array}{lllll}1 & 4.427197 & 0.553549 & 0.175583\end{array}$

$\begin{array}{lllll}6 & 3.580601 & -0.102749 & -0.013700\end{array}$

$\begin{array}{lllll}6 & 1.384756 & -1.783221 & -0.479046\end{array}$

$\begin{array}{lllll}6 & 2.330721 & 0.487461 & -0.393847\end{array}$

$\begin{array}{lllll}6 & 3.707560 & -1.462689 & 0.140239\end{array}$

$\begin{array}{lllll}6 & 2.598173 & -2.316741 & -0.080230\end{array}$

$\begin{array}{lllll}6 & 1.231258 & -0.394730 & -0.639798\end{array}$

$\begin{array}{lllll}6 & 2.153817 & 1.877954 & -0.402082\end{array}$

$\begin{array}{lllll}1 & 4.661777 & -1.888675 & 0.439738\end{array}$

$\begin{array}{lllll}1 & 2.711575 & -3.390389 & 0.043687\end{array}$

$\begin{array}{lllll}1 & 0.341525 & -0.022363 & -1.127647\end{array}$

$\begin{array}{lllll}1 & 0.540089 & -2.435488 & -0.685370\end{array}$

$\begin{array}{lllll}6 & 0.842830 & 2.473254 & -0.423980\end{array}$

$\begin{array}{lllll}1 & 3.001812 & 2.501072 & -0.123393\end{array}$

$\begin{array}{lllll}1 & 0.869531 & 3.564819 & -0.458666\end{array}$

$\begin{array}{lllll}1 & 0.172152 & 2.106340 & -1.207966\end{array}$

$\begin{array}{lllll}1 & -0.858321 & 2.792739 & 0.933038\end{array}$

$\begin{array}{lllll}6 & -0.027587 & 2.085167 & 1.002177\end{array}$

$\begin{array}{llll}6 & -0.471896 & 0.714050 & 1.122918\end{array}$

$\begin{array}{lllll}1 & 0.629378 & 2.388165 & 1.817594\end{array}$

$\begin{array}{llll}6 & -1.691875 & 0.197142 & 0.560598\end{array}$

$\begin{array}{lllll}6 & -2.511331 & 0.940629 & -0.326000\end{array}$

$\begin{array}{lllll}6 & -3.672849 & 0.393398 & -0.863515\end{array}$

$\begin{array}{lllll}6 & -4.066506 & -0.906304 & -0.534922\end{array}$

$\begin{array}{llll}6 & -3.275184 & -1.658456 & 0.342065\end{array}$

$\begin{array}{llll}6 & -2.110796 & -1.121655 & 0.874177\end{array}$

$\begin{array}{lllll}1 & -2.239441 & 1.959374 & -0.587042\end{array}$

$\begin{array}{lllll}1 & -4.280113 & 0.988913 & -1.540844\end{array}$

$\begin{array}{llll}1 & -4.977197 & -1.326979 & -0.951829\end{array}$

$\begin{array}{lllll}1 & -3.571512 & -2.669986 & 0.608721\end{array}$

$\begin{array}{llll}1 & -1.499459 & -1.716019 & 1.549383\end{array}$

$\begin{array}{llll}1 & 0.060864 & 0.059868 & 1.804681\end{array}$

3a) $\underline{A H n-T S D A}$
RB3LYP/6-31G*

$\begin{array}{llll}1 & -1.376161 & 1.324166 & 2.476863\end{array}$

$\begin{array}{llll}6 & -1.342812 & 1.453760 & 1.397413\end{array}$

$\begin{array}{lllll}6 & -1.237727 & 1.766449 & -1.384875\end{array}$

$\begin{array}{llll}6 & -1.773715 & 0.369631 & 0.567770\end{array}$

$\begin{array}{llll}6 & -0.854455 & 2.616731 & 0.852981\end{array}$

$\begin{array}{lllll}6 & -0.780430 & 2.774694 & -0.553112\end{array}$

$\begin{array}{lllll}6 & -1.725630 & 0.565046 & -0.848053\end{array}$

$\begin{array}{llll}6 & -2.115431 & -0.880433 & 1.109907\end{array}$

$\begin{array}{llll}1 & -0.514478 & 3.419738 & 1.501852\end{array}$

$\begin{array}{lllll}1 & -0.393998 & 3.699616 & -0.972434\end{array}$

$\begin{array}{lllll}1 & -2.211576 & -0.142198 & -1.507604\end{array}$

$\begin{array}{lllll}1 & -1.224964 & 1.903331 & -2.463489\end{array}$

$\begin{array}{llll}6 & -2.314669 & -2.022394 & 0.258445\end{array}$

$\begin{array}{llll}1 & -2.032546 & -1.019812 & 2.185554\end{array}$

$\begin{array}{llll}1 & -2.667366 & -2.910674 & 0.786657\end{array}$

$\begin{array}{lllll}1 & -2.973750 & -1.829599 & -0.592084\end{array}$

$1-1.280866 \quad-3.253708$-1.273629

$\begin{array}{lllll}6 & -0.873264 & -2.550457 & -0.543695\end{array}$

$\begin{array}{lllll}6 & -0.040667 & -1.548010 & -1.167664\end{array}$

$\begin{array}{lllll}1 & -0.358295 & -3.109101 & 0.239163\end{array}$

$\begin{array}{llll}1 & -0.173179 & -1.352794 & -2.227913\end{array}$

$\begin{array}{lllll}6 & 1.096238 & -0.941494 & -0.536807\end{array}$

$\begin{array}{llll}6 & 3.351979 & 0.293127 & 0.657349\end{array}$

$\begin{array}{llll}6 & 1.935003 & -0.062180 & -1.270203\end{array}$

$\begin{array}{lllll}6 & 1.433520 & -1.177683 & 0.820583\end{array}$

$\begin{array}{lllll}6 & 2.537000 & -0.562589 & 1.402994\end{array}$

$\begin{array}{lllll}6 & 3.042761 & 0.535500 & -0.686904\end{array}$

$\begin{array}{lllll}1 & 1.695669 & 0.140124 & -2.311587\end{array}$

$\begin{array}{lllll}1 & 0.824658 & -1.846921 & 1.419286\end{array}$

$\begin{array}{llll}1 & 2.770599 & -0.759979 & 2.446472\end{array}$

$1 \quad 3.669820 \quad 1.198956-1.277430$

$\begin{array}{llll}1 & 4.220217 & 0.760665 & 1.113548\end{array}$

\section{4a) $B H x-T S D A$}

RB3LYP/6-31G*

$\begin{array}{llll}1 & 3.954260 & 2.018537 & 0.012460\end{array}$

$\begin{array}{lllll}6 & 3.596134 & 0.992389 & -0.039734\end{array}$

$\begin{array}{lllll}6 & 2.679870 & -1.658704 & -0.187240\end{array}$

$\begin{array}{llll}6 & 2.258370 & 0.764598 & -0.482309\end{array}$

$\begin{array}{llll}6 & 4.418639 & -0.046528 & 0.316787\end{array}$

$\begin{array}{llll}6 & 3.955104 & -1.393148 & 0.230910\end{array}$

$\begin{array}{llll}6 & 1.749317 & -0.591819 & -0.461945\end{array}$

$\begin{array}{llll}6 & 1.363742 & 1.825778 & -0.704491\end{array}$

$\begin{array}{llll}1 & 5.433992 & 0.150240 & 0.650387\end{array}$

$\begin{array}{lllll}1 & 4.633369 & -2.207184 & 0.474091\end{array}$

$\begin{array}{lllll}1 & 0.954590 & -0.832883 & -1.164526\end{array}$

$\begin{array}{lllll}1 & 2.337977 & -2.686584 & -0.284739\end{array}$

$\begin{array}{lllll}6 & 0.011454 & 1.591661 & -0.911835\end{array}$

$\begin{array}{lllll}1 & 1.704215 & 2.835785 & -0.478702\end{array}$

$\begin{array}{lllll}1 & -0.682182 & 2.428188 & -0.945504\end{array}$

$\begin{array}{lllll}1 & -0.314680 & 0.732257 & -1.488361\end{array}$

$\begin{array}{llll}6 & -0.608301 & 0.448226 & 0.856736\end{array}$

$\begin{array}{llll}6 & 0.406761 & -0.521476 & 1.074566\end{array}$

$\begin{array}{lllll}1 & 0.119126 & -1.568431 & 1.031898\end{array}$

$\begin{array}{lllll}1 & 1.138006 & -0.309672 & 1.847834\end{array}$

$\begin{array}{llll}1 & -0.555719 & 1.349277 & 1.459892\end{array}$

$\begin{array}{llll}6 & -1.974715 & 0.078233 & 0.435284\end{array}$

$\begin{array}{lllll}6 & -2.251695 & -1.082847 & -0.311200\end{array}$

$\begin{array}{lllll}6 & -3.553382 & -1.394825 & -0.699924\end{array}$
$6 \begin{array}{llll}6 & -4.615542 & -0.556049 & -0.355578\end{array}$

$\begin{array}{llll}6 & -4.358158 & 0.603092 & 0.379766\end{array}$

$\begin{array}{llll}6 & -3.056203 & 0.917050 & 0.763191\end{array}$

$1-1.441632-1.751670 \quad-0.591015$

$\begin{array}{lllll}1 & -3.738645 & -2.298835 & -1.274767\end{array}$

$1 \quad-5.629892 \quad-0.802080 \quad-0.657316$

$\begin{array}{llll}1 & -5.174431 & 1.265951 & 0.655818\end{array}$

$\begin{array}{llll}1 & -2.866719 & 1.823320 & 1.334642\end{array}$

\section{5a)BHn-TSDA}

RB3LYP/6-31G*

$\begin{array}{llll}1 & -1.848895 & 1.907074 & 1.803025\end{array}$

$\begin{array}{llll}6 & -2.241239 & 1.250955 & 1.028835\end{array}$

$\begin{array}{llll}6 & -3.265763 & -0.448302 & -0.960337\end{array}$

$\begin{array}{llll}6 & -1.737072 & -0.084018 & 0.952989\end{array}$

$\begin{array}{llll}6 & -3.190390 & 1.706525 & 0.148871\end{array}$

$\begin{array}{lllll}6 & -3.715844 & 0.840158 & -0.855489\end{array}$

$\begin{array}{lllll}6 & -2.198480 & -0.930585 & -0.123724\end{array}$

$\begin{array}{llll}6 & -0.658138 & -0.512262 & 1.744902\end{array}$

$\begin{array}{llll}1 & -3.558871 & 2.726224 & 0.222651\end{array}$

$1-4.496752 \quad 1.204154 \quad-1.518406$

$\begin{array}{llll}1 & -2.181108 & -2.002997 & 0.050025\end{array}$

$1-3.692731 \quad-1.119599 \quad-1.701892$

$\begin{array}{llll}6 & 0.001075 & -1.706087 & 1.468741\end{array}$

$\begin{array}{llll}1 & -0.184991 & 0.215888 & 2.400911\end{array}$

$\begin{array}{llll}1 & 0.911817 & -1.945394 & 2.012098\end{array}$

$\begin{array}{llll}1 & -0.564514 & -2.577884 & 1.152323\end{array}$

$\begin{array}{llll}1 & 1.009182 & -2.470736 & -0.584676\end{array}$

$6 \quad 0.660322 \quad-1.440071-0.558470$

$\begin{array}{lllll}6 & -0.501746 & -1.158624 & -1.322859\end{array}$

$\begin{array}{lllll}1 & -0.922298 & -1.974085 & -1.904477\end{array}$

$\begin{array}{lllll}6 & 1.746020 & -0.447072 & -0.395077\end{array}$

$\begin{array}{lllll}6 & 1.511561 & 0.939701 & -0.410657\end{array}$

$\begin{array}{lllll}6 & 2.560778 & 1.843879 & -0.250547\end{array}$

$\begin{array}{lllll}6 & 3.869464 & 1.389273 & -0.074569\end{array}$

$\begin{array}{lllll}6 & 4.117721 & 0.015188 & -0.055996\end{array}$

$\begin{array}{lllll}6 & 3.068370 & -0.888535 & -0.210573\end{array}$

$\begin{array}{lllll}1 & 0.500452 & 1.314246 & -0.538927\end{array}$

$\begin{array}{lllll}1 & 2.353969 & 2.911232 & -0.265012\end{array}$

$\begin{array}{llll}1 & 4.685408 & 2.096778 & 0.045826\end{array}$

$\begin{array}{llll}1 & 5.131268 & -0.354631 & 0.078057\end{array}$

$\begin{array}{lllll}1 & 3.272322 & -1.957451 & -0.194517\end{array}$

$1 \quad-0.574093 \quad-0.193559-1.813244$

6a)TS1 (At)

UB3LYP/6-31G*

$14.648538-2.491371 \quad 0.157553$

$\begin{array}{llll}6 & 4.456971 & -1.422722 & 0.100597\end{array}$

$\begin{array}{lllll}6 & 3.958744 & 1.313249 & -0.052337\end{array}$

$\begin{array}{llll}6 & 3.176805 & -0.942658 & 0.349507\end{array}$

$\begin{array}{lllll}6 & 5.497633 & -0.544387 & -0.221254\end{array}$

$\begin{array}{lllll}6 & 5.237510 & 0.828548 & -0.294468\end{array}$

$\begin{array}{lllll}6 & 2.888770 & 0.444733 & 0.282601\end{array}$

$\begin{array}{llll}1 & 2.386717 & -1.644654 & 0.599470\end{array}$

$1 \quad 6.496831 \quad-0.925023 \quad-0.413447$

$\begin{array}{lllll}1 & 6.037201 & 1.520743 & -0.545631\end{array}$

$\begin{array}{llll}6 & 1.585366 & 0.992042 & 0.538909\end{array}$

$\begin{array}{lllll}1 & 3.764412 & 2.381699 & -0.114481\end{array}$

$\begin{array}{llll}6 & 0.404573 & 0.258549 & 0.809137\end{array}$

$\begin{array}{llll}1 & 1.480418 & 2.062427 & 0.363902\end{array}$ 
$\begin{array}{llll}1 & -6.496829 & 0.925034 & 0.413444\end{array}$

$\begin{array}{lllll}6 & -0.404573 & -0.258558 & -0.809135\end{array}$

$\begin{array}{lllll}6 & -1.585369 & -0.992048 & -0.538906\end{array}$

$\begin{array}{lllll}1 & -1.480423 & -2.062433 & -0.363897\end{array}$

$\begin{array}{lllll}1 & -4.648530 & 2.491374 & -0.157561\end{array}$

$\begin{array}{lllll}6 & -2.888771 & -0.444735 & -0.282600\end{array}$

$\begin{array}{llll}6 & -5.497631 & 0.544394 & 0.221252\end{array}$

$\begin{array}{llll}6 & -3.176802 & 0.942657 & -0.349510\end{array}$

$\begin{array}{lllll}6 & -3.958748 & -1.313247 & 0.052340\end{array}$

$\begin{array}{lllll}6 & -5.237512 & -0.828541 & 0.294469\end{array}$

$\begin{array}{lllll}6 & -4.456967 & 1.422725 & -0.100601\end{array}$

$\begin{array}{llll}1 & -2.386712 & 1.644650 & -0.599475\end{array}$

$\begin{array}{llll}1 & -3.764418 & -2.381697 & 0.114487\end{array}$

$\begin{array}{llll}1 & -6.037205 & -1.520733 & 0.545635\end{array}$

$\begin{array}{lllll}1 & -0.526744 & 0.711064 & -1.288272\end{array}$

$\begin{array}{lllll}1 & 0.411353 & -0.827660 & -1.251812\end{array}$

$\begin{array}{llll}1 & -0.411354 & 0.827649 & 1.251816\end{array}$

$\begin{array}{llll}1 & 0.526746 & -0.711073 & 1.288274\end{array}$

\section{7a) TS1 (Ac)}

\section{UB3LYP/6-31G*}

$\begin{array}{llll}1 & 2.586383 & 2.886780 & 0.003753\end{array}$

$\begin{array}{llll}6 & 2.993910 & 1.878888 & 0.007558\end{array}$

$\begin{array}{lllll}6 & 4.033293 & -0.705614 & 0.019523\end{array}$

$\begin{array}{llll}6 & 2.162192 & 0.805118 & 0.302627\end{array}$

$\begin{array}{lllll}6 & 4.347154 & 1.673331 & -0.281990\end{array}$

$\begin{array}{lllll}6 & 4.860270 & 0.371305 & -0.271666\end{array}$

$\begin{array}{lllll}6 & 2.658750 & -0.523937 & 0.318373\end{array}$

$\begin{array}{llll}1 & 1.118172 & 0.991433 & 0.536664\end{array}$

$\begin{array}{lllll}1 & 4.992751 & 2.516027 & -0.512797\end{array}$

$\begin{array}{lllll}1 & 5.910099 & 0.199149 & -0.495114\end{array}$

$\begin{array}{llll}6 & 1.841415 & -1.664087 & 0.626525\end{array}$

$\begin{array}{lllll}1 & 4.439793 & -1.714537 & 0.023311\end{array}$

$\begin{array}{lllll}6 & 0.439489 & -1.657204 & 0.829717\end{array}$

$\begin{array}{llll}1 & 2.332708 & -2.633333 & 0.549160\end{array}$

$\begin{array}{llll}1 & -4.992784 & 2.516015 & 0.512743\end{array}$

$\begin{array}{lllll}6 & -0.439471 & -1.657190 & -0.829697\end{array}$

$\begin{array}{lllll}6 & -1.841396 & -1.664085 & -0.626498\end{array}$

$\begin{array}{lllll}1 & -2.332684 & -2.633334 & -0.549121\end{array}$

$\begin{array}{lllll}1 & -2.586386 & 2.886772 & -0.003670\end{array}$

$\begin{array}{lllll}6 & -2.658742 & -0.523938 & -0.318360\end{array}$

$\begin{array}{lllll}6 & -4.347176 & 1.673322 & 0.281956\end{array}$

$\begin{array}{lllll}6 & -2.162184 & 0.805116 & -0.302563\end{array}$

$\begin{array}{llll}6 & -4.033300 & -0.705618 & -0.019578\end{array}$

$\begin{array}{llll}6 & -4.860291 & 0.371296 & 0.271586\end{array}$

$\begin{array}{lllll}6 & -2.993916 & 1.878881 & -0.007518\end{array}$

$\begin{array}{lllll}1 & -1.118150 & 0.991435 & -0.536531\end{array}$

$\begin{array}{lllll}1 & -4.439800 & -1.714541 & -0.023399\end{array}$

$\begin{array}{llll}1 & -5.910131 & 0.199136 & 0.494978\end{array}$

$\begin{array}{llll}1 & -0.000068 & -0.755350 & -1.254949\end{array}$

$\begin{array}{lllll}1 & -0.034634 & -2.556875 & -1.288580\end{array}$

$\begin{array}{llll}1 & 0.000082 & -0.755374 & 1.254984\end{array}$

$\begin{array}{lllll}1 & 0.034658 & -2.556899 & 1.288588\end{array}$

\section{8a) $\underline{T S 1(G s)}$}

UB3LYP/6-31G*

$\begin{array}{llll}1 & -4.461506 & 0.652438 & -1.714084\end{array}$

$\begin{array}{lllll}6 & -3.851712 & 0.152138 & -0.965833\end{array}$

$\begin{array}{lllll}6 & -2.281983 & -1.123470 & 0.948701\end{array}$

$\begin{array}{lllll}6 & -2.784950 & 0.831206 & -0.386887\end{array}$
$6-4.146496-1.162632 \quad-0.591989$

$\begin{array}{llll}6 & -3.352572 & -1.794224 & 0.372483\end{array}$

$\begin{array}{llll}6 & -1.965663 & 0.212348 & 0.591476\end{array}$

$\begin{array}{llll}1 & -2.584598 & 1.855211 & -0.688485\end{array}$

$1-4.982746 \quad-1.687781-1.044890$

$\begin{array}{llll}1 & -3.570905 & -2.816429 & 0.671319\end{array}$

$\begin{array}{llll}6 & -0.848154 & 0.869787 & 1.215809\end{array}$

$\begin{array}{lllll}1 & -1.665909 & -1.624161 & 1.692201\end{array}$

$\begin{array}{llll}6 & -0.402787 & 2.187225 & 0.939047\end{array}$

$\begin{array}{llll}1 & -0.256496 & 0.262774 & 1.897129\end{array}$

$\begin{array}{llll}1 & 3.622778 & -3.177558 & 0.049303\end{array}$

$\begin{array}{lllll}6 & 0.634121 & 2.313851 & -0.610033\end{array}$

$\begin{array}{lllll}6 & 1.968877 & 1.886869 & -0.405949\end{array}$

$\begin{array}{lllll}1 & 2.703170 & 2.640379 & -0.124206\end{array}$

$\begin{array}{lllll}1 & 1.266519 & -2.698329 & -0.588415\end{array}$

$\begin{array}{lllll}6 & 2.395863 & 0.525252 & -0.323938\end{array}$

$\begin{array}{lllll}6 & 3.281799 & -2.150950 & -0.052897\end{array}$

$\begin{array}{lllll}6 & 1.517126 & -0.566487 & -0.551073\end{array}$

$\begin{array}{llll}6 & 3.736580 & 0.222225 & 0.036500\end{array}$

$\begin{array}{lllll}6 & 4.170336 & -1.087549 & 0.165207\end{array}$

$\begin{array}{lllll}6 & 1.959463 & -1.880008 & -0.409681\end{array}$

$1 \quad 0.493630 \quad-0.382201-0.856522$

$\begin{array}{lllll}1 & 4.426826 & 1.043310 & 0.217540\end{array}$

$1 \quad 5.202075 \quad-1.290452 \quad 0.441093$

$\begin{array}{llll}1 & 0.011052 & 1.715246 & -1.274900\end{array}$

$\begin{array}{lllll}1 & 0.534273 & 3.378028 & -0.824554\end{array}$

$\begin{array}{llll}1 & 0.257235 & 2.620915 & 1.687019\end{array}$

$\begin{array}{llll}1 & -1.174494 & 2.895134 & 0.634789\end{array}$

\section{9a)TS1(Gu)}

UB3LYP/6-31G*

$\begin{array}{llll}1 & -2.310878 & -1.206908 & 2.577822\end{array}$

$\begin{array}{lllll}6 & -2.303683 & -1.034251 & 1.504439\end{array}$

$\begin{array}{lllll}6 & -2.279743 & -0.590463 & -1.246034\end{array}$

$\begin{array}{llll}6 & -1.865947 & 0.190060 & 1.010323\end{array}$

$\begin{array}{llll}6 & -2.737719 & -2.038697 & 0.634990\end{array}$

$\begin{array}{lllll}6 & -2.725010 & -1.805163 & -0.746301\end{array}$

$\begin{array}{llll}6 & -1.836149 & 0.445922 & -0.384259\end{array}$

$\begin{array}{llll}1 & -1.540929 & 0.957061 & 1.705668\end{array}$

$\begin{array}{lllll}1 & -3.084986 & -2.990789 & 1.026634\end{array}$

$\begin{array}{lllll}1 & -3.060483 & -2.579867 & -1.431212\end{array}$

$\begin{array}{lllll}6 & -1.384977 & 1.690754 & -0.935863\end{array}$

$\begin{array}{llll}1 & -2.263910 & -0.419232 & -2.320177\end{array}$

$\begin{array}{llll}6 & -0.914500 & 2.797339 & -0.187790\end{array}$

$\begin{array}{lllll}1 & -1.335415 & 1.749215 & -2.022102\end{array}$

$\begin{array}{llll}1 & 3.076122 & -2.997418 & -1.025879\end{array}$

$\begin{array}{llll}6 & 0.920990 & 2.796565 & 0.187055\end{array}$

$\begin{array}{llll}6 & 1.390285 & 1.689696 & 0.935442\end{array}$

$\begin{array}{llll}1 & 1.341815 & 1.748919 & 2.021690\end{array}$

$1 \quad 2.305317 \quad-1.212401 \quad-2.577388$

$\begin{array}{lllll}6 & 1.837916 & 0.443471 & 0.384177\end{array}$

$\begin{array}{lllll}6 & 2.731818 & -2.044151 & -0.634474\end{array}$

$\begin{array}{lllll}6 & 1.865668 & 0.186711 & -1.010292\end{array}$

$\begin{array}{llll}6 & 2.279663 & -0.593558 & 1.246141\end{array}$

$\begin{array}{llll}6 & 2.721170 & -1.809739 & 0.746698\end{array}$

$6 \quad 2.299633 \quad-1.039078 \quad-1.504103$

$\begin{array}{lllll}1 & 1.542165 & 0.954208 & -1.705786\end{array}$

$\begin{array}{llll}1 & 2.265353 & -0.421628 & 2.320195\end{array}$

$\begin{array}{llll}1 & 3.055275 & -2.584924 & 1.431733\end{array}$

$\begin{array}{lllll}1 & 1.313773 & 2.896870 & -0.823583\end{array}$

$\begin{array}{lllll}1 & 0.951998 & 3.747647 & 0.718892\end{array}$ $\begin{array}{llll}1 & -1.307481 & 2.898525 & 0.822677\end{array}$

$\begin{array}{llll}1 & -0.944238 & 3.748213 & -0.720076\end{array}$

10a) $\underline{T S 1}(\mathrm{Gw})$

UB3LYP/6-31G*

$1 \quad-5.003715 \quad-2.017386-1.005407$

$\begin{array}{lllll}6 & -4.640985 & -1.095883 & -0.556766\end{array}$

$\begin{array}{llll}6 & -3.705052 & 1.263526 & 0.588858\end{array}$

$\begin{array}{lllll}6 & -3.325518 & -1.021439 & -0.115382\end{array}$

$\begin{array}{lllll}6 & -5.500093 & 0.001216 & -0.427026\end{array}$

$\begin{array}{llll}6 & -5.020591 & 1.181666 & 0.151292\end{array}$

$\begin{array}{llll}6 & -2.815478 & 0.164742 & 0.472768\end{array}$

$\begin{array}{lllll}1 & -2.681074 & -1.888991 & -0.223751\end{array}$

$\begin{array}{llll}1 & -6.528323 & -0.06357 & -0.771635\end{array}$

$\begin{array}{llll}1 & -5.677572 & 2.041315 & 0.256803\end{array}$

$\begin{array}{llll}6 & -1.46374 & 0.297574 & 0.940765\end{array}$

$\begin{array}{llll}1 & -3.339503 & 2.186275 & 1.033925\end{array}$

$\begin{array}{llll}6 & -0.439157 & -0.674057 & 0.836691\end{array}$

$\begin{array}{llll}1 & -1.184011 & 1.287322 & 1.299453\end{array}$

$\begin{array}{llll}1 & 6.528359 & -0.063585 & 0.771511\end{array}$

$\begin{array}{lllll}6 & 0.439147 & -0.674045 & -0.836598\end{array}$

$\begin{array}{lllll}6 & 1.463709 & 0.297603 & -0.940673\end{array}$

$11.18395 \quad 1.287365 \quad-1.299295$

$\begin{array}{llll}1 & 5.00376 & -2.017407 & 1.005298\end{array}$

$\begin{array}{lllll}6 & 2.815468 & 0.164756 & -0.472747\end{array}$

$\begin{array}{llll}6 & 5.500117 & 0.001208 & 0.42694\end{array}$

$\begin{array}{llll}6 & 3.32553 & -1.02144 & 0.115357\end{array}$

$\begin{array}{llll}6 & 3.705038 & 1.263542 & -0.588848\end{array}$

$\begin{array}{llll}6 & 5.020594 & 1.18167 & -0.151336\end{array}$

$\begin{array}{lllll}6 & 4.641013 & -1.095894 & 0.55669\end{array}$

$\begin{array}{llll}1 & 2.681089 & -1.888992 & 0.223736\end{array}$

$\begin{array}{lllll}1 & 3.339472 & 2.186302 & -1.03388\end{array}$

$\begin{array}{lllll}1 & 5.677571 & 2.041321 & -0.256854\end{array}$

$\begin{array}{llll}1 & 0.770494 & -1.7125 & -0.819327\end{array}$

$\begin{array}{lllll}1 & -0.412307 & -0.536714 & -1.50054\end{array}$

$\begin{array}{llll}1 & -0.770486 & -1.712518 & 0.819404\end{array}$

$\begin{array}{llll}1 & 0.412286 & -0.536712 & 1.500642\end{array}$

11a) $M 2 \cdot(A t)$

UB3LYP/6-31G*

$1 \quad-4.676287 \quad-2.497134-0.117796$

$\begin{array}{llll}6 & -4.486270 & -1.427460 & -0.076142\end{array}$

$\begin{array}{llll}6 & -4.001413 & 1.317379 & 0.035843\end{array}$

$\begin{array}{llll}6 & -3.198490 & -0.953865 & -0.284378\end{array}$

$\begin{array}{llll}6 & -5.540369 & -0.543029 & 0.186448\end{array}$

$\begin{array}{llll}6 & -5.284116 & 0.834409 & 0.239733\end{array}$

$\begin{array}{lllll}6 & -2.909509 & 0.441187 & -0.236468\end{array}$

$\begin{array}{lllll}1 & -2.398536 & -1.659666 & -0.486291\end{array}$

$\begin{array}{lllll}1 & -6.545999 & -0.920779 & 0.347694\end{array}$

$\begin{array}{llll}1 & -6.094905 & 1.529192 & 0.444033\end{array}$

$\begin{array}{lllll}6 & -1.612995 & 0.974458 & -0.448360\end{array}$

$\begin{array}{llll}1 & -3.810640 & 2.387269 & 0.080025\end{array}$

$\begin{array}{llll}6 & -0.367524 & 0.192523 & -0.675742\end{array}$

$\begin{array}{lllll}1 & -1.503688 & 2.052247 & -0.335246\end{array}$

$1 \quad 6.545998 \quad 0.920780 \quad-0.347701$

$\begin{array}{lllll}6 & 0.367525 & -0.192524 & 0.675747\end{array}$

$\begin{array}{llll}6 & 1.612996 & -0.974458 & 0.448364\end{array}$

$\begin{array}{lllll}1 & 1.503690 & -2.052247 & 0.335252\end{array}$

$\begin{array}{lllll}1 & 4.676286 & 2.497134 & 0.117790\end{array}$

$\begin{array}{lllll}6 & 2.909510 & -0.441187 & 0.236469\end{array}$ 
$\begin{array}{lllll}6 & 5.540368 & 0.543029 & -0.186453\end{array}$

$\begin{array}{llll}6 & 3.198490 & 0.953865 & 0.284377\end{array}$

$\begin{array}{lllll}6 & 4.001413 & -1.317379 & -0.035842\end{array}$

$\begin{array}{lllll}6 & 5.284115 & -0.834409 & -0.239735\end{array}$

$\begin{array}{lllll}6 & 4.486269 & 1.427460 & 0.076138\end{array}$

$\begin{array}{llll}1 & 2.398536 & 1.659666 & 0.486291\end{array}$

$\begin{array}{lllll}1 & 3.810640 & -2.387269 & -0.080022\end{array}$

$\begin{array}{llll}1 & 6.094904 & -1.529192 & -0.444036\end{array}$

$\begin{array}{llll}1 & 0.565099 & 0.733235 & 1.227864\end{array}$

$\begin{array}{llll}1 & -0.338734 & -0.775346 & 1.278308\end{array}$

$\begin{array}{lllll}1 & 0.338735 & 0.775346 & -1.278303\end{array}$

$\begin{array}{llll}1 & -0.565098 & -0.733235 & -1.227860\end{array}$

12a) $M 2 \cdot(A c)$

UB3LYP/6-31G*

$1 \quad-2.646486 \quad 2.890595 \quad-0.233714$

$\begin{array}{llll}6 & -3.049854 & 1.884878 & -0.144922\end{array}$

$\begin{array}{lllll}6 & -4.087824 & -0.694368 & 0.083307\end{array}$

$\begin{array}{lllll}6 & -2.213015 & 0.794143 & -0.335142\end{array}$

$\begin{array}{llll}6 & -4.405234 & 1.702819 & 0.158397\end{array}$

$\begin{array}{llll}6 & -4.915316 & 0.401545 & 0.269304\end{array}$

$\begin{array}{lllll}6 & -2.704495 & -0.539751 & -0.227035\end{array}$

$\begin{array}{lllll}1 & -1.167407 & 0.961931 & -0.575432\end{array}$

$\begin{array}{llll}1 & -5.054383 & 2.561175 & 0.306045\end{array}$

$\begin{array}{llll}1 & -5.965804 & 0.249207 & 0.504031\end{array}$

$\begin{array}{lllll}6 & -1.891425 & -1.685413 & -0.419170\end{array}$

$\begin{array}{lllll}1 & -4.491238 & -1.700508 & 0.172349\end{array}$

$\begin{array}{lllll}6 & -0.424539 & -1.683958 & -0.670013\end{array}$

$1-2.367138-2.653903-0.271504$

$\begin{array}{lllll}1 & 5.054613 & 2.560988 & -0.306096\end{array}$

$\begin{array}{lllll}6 & 0.424397 & -1.683757 & 0.670066\end{array}$

$\begin{array}{llll}6 & 1.891280 & -1.685305 & 0.419211\end{array}$

$\begin{array}{llll}1 & 2.366910 & -2.653839 & 0.271551\end{array}$

$\begin{array}{llll}1 & 2.646743 & 2.890634 & 0.233657\end{array}$

$\begin{array}{llll}6 & 2.704445 & -0.539717 & 0.227053\end{array}$

$\begin{array}{lllll}6 & 4.405384 & 1.702694 & -0.158434\end{array}$

$\begin{array}{llll}6 & 2.213085 & 0.794224 & 0.335130\end{array}$

$\begin{array}{lllll}6 & 4.087757 & -0.694463 & -0.083308\end{array}$

$\begin{array}{lllll}6 & 4.915346 & 0.401370 & -0.269329\end{array}$

$\begin{array}{llll}6 & 3.050023 & 1.884880 & 0.144885\end{array}$

$\begin{array}{llll}1 & 1.167492 & 0.962116 & 0.575408\end{array}$

$14.491074-1.700642 \quad-0.172346$

$\begin{array}{llll}1 & 5.965819 & 0.248934 & -0.504064\end{array}$

$\begin{array}{llll}1 & 0.113860 & -0.814224 & 1.259971\end{array}$

$\begin{array}{llll}1 & 0.140851 & -2.573205 & 1.244602\end{array}$

$\begin{array}{llll}1 & -0.113991 & -0.814607 & -1.260181\end{array}$

$1-0.141002-2.573589-1.244270$

13a)・M2 $(G s)$

UB3LYP/6-31G*

$\begin{array}{llll}1 & -4.645131 & 0.394397 & -1.704654\end{array}$

$\begin{array}{lllll}6 & -3.993022 & -0.011853 & -0.935336\end{array}$

$\begin{array}{llll}6 & -2.318883 & -1.057116 & 1.035131\end{array}$

$\begin{array}{lllll}6 & -2.916637 & 0.740654 & -0.485851\end{array}$

$\begin{array}{lllll}6 & -4.246974 & -1.284512 & -0.408511\end{array}$

$\begin{array}{llll}6 & -3.398148 & -1.798728 & 0.581905\end{array}$

$\begin{array}{llll}6 & -2.037890 & 0.243283 & 0.520451\end{array}$

$\begin{array}{lllll}1 & -2.744323 & 1.724881 & -0.910875\end{array}$

$\begin{array}{lllll}1 & -5.092483 & -1.866690 & -0.763775\end{array}$

$\begin{array}{llll}1 & -3.585010 & -2.785937 & 0.997129\end{array}$ $\begin{array}{llll}6 & -0.924006 & 0.969310 & 1.017430\end{array}$

$\begin{array}{llll}1 & -1.663587 & -1.464600 & 1.801389\end{array}$

$\begin{array}{llll}6 & -0.484214 & 2.316812 & 0.565012\end{array}$

$\begin{array}{llll}1 & -0.303932 & 0.467079 & 1.756849\end{array}$

$\begin{array}{lllll}1 & 3.883577 & -3.127732 & -0.162390\end{array}$

$\begin{array}{lllll}6 & 0.598532 & 2.303534 & -0.595508\end{array}$

$\begin{array}{lllll}6 & 1.953080 & 1.852504 & -0.176979\end{array}$

$\begin{array}{llll}1 & 2.610711 & 2.610302 & 0.246747\end{array}$

$\begin{array}{llll}1 & 1.583691 & -2.695930 & -1.012150\end{array}$

$\begin{array}{lllll}6 & 2.438743 & 0.522636 & -0.189835\end{array}$

$\begin{array}{lllll}6 & 3.484024 & -2.117517 & -0.168982\end{array}$

$\begin{array}{llll}6 & 1.673109 & -0.583297 & -0.663509\end{array}$

$\begin{array}{llll}6 & 3.752553 & 0.241962 & 0.291773\end{array}$

$\begin{array}{llll}6 & 4.260038 & -1.046643 & 0.299562\end{array}$

$\begin{array}{llll}6 & 2.191255 & -1.871632 & -0.647073\end{array}$

$1 \quad 0.668797 \quad-0.418564 \quad-1.038688$

$\begin{array}{llll}1 & 4.359159 & 1.067147 & 0.658156\end{array}$

$1 \quad 5.265147 \quad-1.227895 \quad 0.672163$

$\begin{array}{lllll}1 & 0.200420 & 1.695020 & -1.415358\end{array}$

$\begin{array}{lllll}1 & 0.665217 & 3.334186 & -0.966518\end{array}$

$\begin{array}{llll}1 & -0.057618 & 2.874746 & 1.407301\end{array}$

$\begin{array}{lllll}1 & -1.333993 & 2.903208 & 0.196589\end{array}$

\section{4a) $\underline{M 2 \cdot(G n)}$}

UB3LYP/6-31G*

$\begin{array}{llll}1 & -2.485403 & -1.358398 & 2.541931\end{array}$

$\begin{array}{llll}6 & -2.495979 & -1.112566 & 1.482906\end{array}$

$\begin{array}{llll}6 & -2.517196 & -0.489237 & -1.235348\end{array}$

$\begin{array}{llll}6 & -1.953997 & 0.091889 & 1.056377\end{array}$

$\begin{array}{llll}6 & -3.054099 & -2.011617 & 0.565726\end{array}$

$\begin{array}{lllll}6 & -3.058815 & -1.686829 & -0.798602\end{array}$

$\begin{array}{lllll}6 & -1.945178 & 0.447504 & -0.323761\end{array}$

$\begin{array}{llll}1 & -1.522891 & 0.769105 & 1.786362\end{array}$

$\begin{array}{lllll}1 & -3.478636 & -2.951330 & 0.907509\end{array}$

$1-3.488145-2.378436-1.519211$

$\begin{array}{lllll}6 & -1.412229 & 1.666360 & -0.810694\end{array}$

$\begin{array}{lllll}1 & -2.523457 & -0.245280 & -2.295355\end{array}$

$\begin{array}{lllll}6 & -0.789894 & 2.751960 & -0.006289\end{array}$

$\begin{array}{lllll}1 & -1.429377 & 1.811664 & -1.890272\end{array}$

$1 \quad 3.471946 \quad-2.956670 \quad-0.906069$

$\begin{array}{llll}6 & 0.794264 & 2.752053 & 0.004935\end{array}$

$\begin{array}{llll}6 & 1.416634 & 1.666802 & 0.809807\end{array}$

$\begin{array}{llll}1 & 1.435933 & 1.813558 & 1.889145\end{array}$

$\begin{array}{lllll}1 & 2.478991 & -1.363904 & -2.540822\end{array}$

$\begin{array}{llll}6 & 1.946603 & 0.446387 & 0.323573\end{array}$

$\begin{array}{lllll}6 & 3.049646 & -2.015784 & -0.564739\end{array}$

$\begin{array}{lllll}6 & 1.952565 & 0.089108 & -1.056151\end{array}$

$\begin{array}{llll}6 & 2.518423 & -0.490268 & 1.235372\end{array}$

$\begin{array}{llll}6 & 3.057163 & -1.689362 & 0.799192\end{array}$

$\begin{array}{lllll}6 & 2.491682 & -1.116835 & -1.482109\end{array}$

$\begin{array}{lllll}1 & 1.521560 & 0.766281 & -1.786227\end{array}$

$\begin{array}{llll}1 & 2.526834 & -0.245047 & 2.295073\end{array}$

$1 \quad 3.486417 \quad-2.380879 \quad 1.519933$

$\begin{array}{lllll}1 & 1.142400 & 2.738818 & -1.033593\end{array}$

$\begin{array}{llll}1 & 1.094040 & 3.722111 & 0.423888\end{array}$

$\begin{array}{llll}1 & -1.138052 & 2.739176 & 1.032216\end{array}$

$\begin{array}{lllll}1 & -1.089723 & 3.721842 & -0.425674\end{array}$

$15 a) \cdot M 2 \cdot(G w)$

UB3LYP/6-31G*
$1 \quad-5.283610 \quad-1.836698 \quad 0.799363$

$\begin{array}{llll}6 & -4.777717 & -0.950446 & 0.424205\end{array}$

$\begin{array}{lllll}6 & -3.481874 & 1.325725 & -0.533864\end{array}$

$\begin{array}{llll}6 & -3.426594 & -1.015671 & 0.114962\end{array}$

$\begin{array}{llll}6 & -5.494234 & 0.242053 & 0.258637\end{array}$

$\begin{array}{lllll}6 & -4.831266 & 1.379306 & -0.224272\end{array}$

$\begin{array}{llll}6 & -2.726360 & 0.125022 & -0.377496\end{array}$

$\begin{array}{llll}1 & -2.895853 & -1.952486 & 0.254152\end{array}$

$\begin{array}{llll}1 & -6.552070 & 0.284852 & 0.501810\end{array}$

$\begin{array}{lllll}1 & -5.377304 & 2.310034 & -0.356413\end{array}$

$\begin{array}{lllll}6 & -1.348474 & 0.117202 & -0.707395\end{array}$

$\begin{array}{lllll}1 & -2.975218 & 2.212523 & -0.907839\end{array}$

$\begin{array}{lllll}6 & -0.410232 & -1.034692 & -0.577532\end{array}$

$\begin{array}{lllll}1 & -0.919692 & 1.062943 & -1.034394\end{array}$

$\begin{array}{llll}1 & 6.486582 & 0.273880 & -0.751165\end{array}$

$\begin{array}{llll}6 & 0.440425 & -1.031809 & 0.750754\end{array}$

$\begin{array}{llll}6 & 1.395938 & 0.108102 & 0.867816\end{array}$

$\begin{array}{llll}1 & 1.006141 & 1.040531 & 1.272109\end{array}$

$1 \quad 5.165771 \quad-1.816504 \quad-1.040749$

$\begin{array}{lllll}6 & 2.743126 & 0.118604 & 0.428547\end{array}$

$\begin{array}{lllll}6 & 5.451316 & 0.232192 & -0.424872\end{array}$

$6 \quad 3.382896 \quad-1.006159-0.170868$

$\begin{array}{llll}6 & 3.526820 & 1.301618 & 0.577692\end{array}$

$\begin{array}{llll}6 & 4.847439 & 1.353507 & 0.161740\end{array}$

$\begin{array}{lllll}6 & 4.705570 & -0.942782 & -0.585869\end{array}$

$\begin{array}{lllll}1 & 2.827398 & -1.928959 & -0.307750\end{array}$

$\begin{array}{llll}1 & 3.065838 & 2.175946 & 1.031616\end{array}$

$\begin{array}{llll}1 & 5.416942 & 2.270467 & 0.290989\end{array}$

$\begin{array}{llll}1 & -0.261089 & -1.014442 & 1.593727\end{array}$

$\begin{array}{llll}1 & 0.969878 & -1.991396 & 0.797818\end{array}$

$1 \quad 0.295149-1.033052-1.418458$

$\begin{array}{lllll}1 & -0.949535 & -1.988270 & -0.617917\end{array}$

\section{6a)TS2x}

UB3LYP/6-31G*

$\begin{array}{llll}1 & 4.434052 & 0.317266 & 0.220894\end{array}$

$\begin{array}{llll}6 & 3.535490 & -0.257272 & 0.006956\end{array}$

$\begin{array}{lllll}6 & 1.203535 & -1.732387 & -0.536292\end{array}$

$\begin{array}{lllll}6 & 2.343872 & 0.453733 & -0.369756\end{array}$

$\begin{array}{llll}6 & 3.535860 & -1.622689 & 0.132942\end{array}$

$\begin{array}{lllll}6 & 2.354454 & -2.373274 & -0.126357\end{array}$

$\begin{array}{lllll}6 & 1.163431 & -0.323196 & -0.652367\end{array}$

$\begin{array}{lllll}6 & 2.272447 & 1.842102 & -0.338893\end{array}$

$14.442309-2.140606 \quad 0.435914$

$\begin{array}{lllll}1 & 2.374704 & -3.455423 & -0.028135\end{array}$

$1 \quad 0.312995 \quad-2.305182 \quad-0.781331$

$\begin{array}{llll}6 & 0.967226 & 2.520300 & -0.333226\end{array}$

$\begin{array}{llll}1 & 3.160951 & 2.408302 & -0.066710\end{array}$

$\begin{array}{lllll}1 & 1.077112 & 3.608446 & -0.298423\end{array}$

$\begin{array}{lllll}1 & 0.350553 & 2.280282 & -1.209837\end{array}$

$\begin{array}{llll}1 & -0.773888 & 2.747038 & 0.953307\end{array}$

$\begin{array}{lllll}6 & 0.094206 & 2.078097 & 0.955233\end{array}$

$\begin{array}{llll}6 & -0.343590 & 0.661872 & 1.016865\end{array}$

$\begin{array}{llll}1 & 0.694222 & 2.329315 & 1.833757\end{array}$

$\begin{array}{llll}6 & -1.616815 & 0.198872 & 0.517446\end{array}$

$\begin{array}{lllll}6 & -2.425047 & 0.964267 & -0.358803\end{array}$

$\begin{array}{lllll}6 & -3.631695 & 0.468023 & -0.844071\end{array}$

$\begin{array}{llll}6 & -4.079617 & -0.802453 & -0.472416\end{array}$

$\begin{array}{llll}6 & -3.298304 & -1.576150 & 0.393855\end{array}$

$\begin{array}{lllll}6 & -2.090318 & -1.088180 & 0.875977\end{array}$

$\begin{array}{lllll}1 & -2.105974 & 1.959247 & -0.656149\end{array}$ 
$\begin{array}{llll}1 & -4.230623 & 1.079688 & -1.514373\end{array}$

$\begin{array}{lllll}1 & -5.024867 & -1.183694 & -0.848472\end{array}$

$\begin{array}{llll}1 & -3.637318 & -2.564516 & 0.694328\end{array}$

$\begin{array}{llll}1 & -1.489201 & -1.697439 & 1.546958\end{array}$

$\begin{array}{llll}1 & 0.132693 & 0.027876 & 1.757445\end{array}$

$\begin{array}{lllll}1 & 0.371470 & 0.119413 & -1.241735\end{array}$

17a) $\underline{T S 2 n}$

UB3LYP/6-31G*

$\begin{array}{llll}1 & -2.198249 & 1.471833 & 2.211073\end{array}$

$\begin{array}{llll}6 & -1.824191 & 1.516918 & 1.190610\end{array}$

$\begin{array}{lllll}6 & -0.859798 & 1.622125 & -1.448098\end{array}$

$\begin{array}{llll}6 & -2.033356 & 0.380362 & 0.336518\end{array}$

$\begin{array}{llll}6 & -1.131252 & 2.615163 & 0.751230\end{array}$

$\begin{array}{lllll}6 & -0.625937 & 2.667248 & -0.578274\end{array}$

$\begin{array}{lllll}6 & -1.535189 & 0.460698 & -1.013455\end{array}$

$\begin{array}{llll}6 & -2.578197 & -0.809436 & 0.807060\end{array}$

$\begin{array}{llll}1 & -0.962168 & 3.455333 & 1.419804\end{array}$

$\begin{array}{lllll}1 & -0.087045 & 3.549439 & -0.912820\end{array}$

$\begin{array}{lllll}1 & -0.522783 & 1.686590 & -2.479737\end{array}$

$\begin{array}{lllll}6 & -2.441862 & -2.045316 & 0.015923\end{array}$

$\begin{array}{llll}1 & -2.893254 & -0.874466 & 1.846279\end{array}$

$\begin{array}{llll}1 & -2.938377 & -2.893478 & 0.497174\end{array}$

$\begin{array}{lllll}1 & -2.866290 & -1.953861 & -0.992889\end{array}$

$\begin{array}{lllll}1 & -0.919307 & -3.384811 & -0.742759\end{array}$

$\begin{array}{lllll}6 & -0.895412 & -2.444339 & -0.176946\end{array}$

$\begin{array}{llll}6 & -0.017968 & -1.479817 & -0.890316\end{array}$

$\begin{array}{lllll}1 & -0.504667 & -2.682231 & 0.815543\end{array}$

$\begin{array}{llll}6 & 1.177759 & -0.898885 & -0.331253\end{array}$

$\begin{array}{lllll}6 & 1.457437 & -0.874589 & 1.057089\end{array}$

$\begin{array}{llll}6 & 2.625127 & -0.298768 & 1.545671\end{array}$

$\begin{array}{llll}6 & 3.559870 & 0.269833 & 0.674872\end{array}$

$\begin{array}{lllll}6 & 3.302263 & 0.264060 & -0.699873\end{array}$

$\begin{array}{lllll}6 & 2.133376 & -0.302934 & -1.191948\end{array}$

$\begin{array}{llll}1 & 0.746691 & -1.300908 & 1.757920\end{array}$

$\begin{array}{lllll}1 & 2.810934 & -0.295458 & 2.616985\end{array}$

$\begin{array}{llll}1 & 4.474614 & 0.710544 & 1.061505\end{array}$

$\begin{array}{lllll}1 & 4.018714 & 0.703278 & -1.389783\end{array}$

$\begin{array}{lllll}1 & 1.944549 & -0.304021 & -2.263290\end{array}$

$\begin{array}{llll}1 & -1.938129 & -0.210851 & -1.761068\end{array}$

$\begin{array}{lllll}1 & -0.041253 & -1.530868 & -1.976801\end{array}$

\section{8a)TScisDCB}

UB3LYP/6-31G*

$\begin{array}{lrrrr}6 & 3.665030 & -1.736357 & -0.192068\end{array}$

$\begin{array}{llll}6 & -1.349493 & 1.565953 & 0.830059\end{array}$

$\begin{array}{lllll}6 & -1.026730 & 2.717164 & -0.098775\end{array}$

$\begin{array}{llll}6 & 1.126678 & 1.660783 & 0.221583\end{array}$

$\begin{array}{llll}6 & 0.267902 & 2.319992 & -0.827626\end{array}$

$\begin{array}{lllll}1 & 0.024001 & 1.606786 & -1.621627\end{array}$

$\begin{array}{llll}1 & 0.769808 & 3.172702 & -1.316282\end{array}$

$\begin{array}{llll}1 & -1.207588 & 1.718844 & 1.896450\end{array}$

$\begin{array}{lllll}1 & 1.256038 & 2.222587 & 1.145074\end{array}$

$\begin{array}{llll}6 & 1.968040 & 0.525508 & 0.050812\end{array}$

$\begin{array}{llll}1 & -0.857669 & 3.622297 & 0.496873\end{array}$

$\begin{array}{lllll}1 & -1.844292 & 2.948720 & -0.795673\end{array}$

$\begin{array}{lllll}1 & 1.383934 & 0.036037 & -1.985688\end{array}$

$\begin{array}{llll}6 & 2.011237 & -0.242818 & -1.145190\end{array}$

$\begin{array}{llll}6 & 2.812062 & 0.107912 & 1.118876\end{array}$

$\begin{array}{llll}1 & 4.272724 & -1.290172 & 1.832626\end{array}$ $\begin{array}{llll}6 & 3.639457 & -0.997778 & 0.998738\end{array}$

$\begin{array}{lllll}6 & 2.845524 & -1.347565 & -1.257955\end{array}$

$\begin{array}{lllll}1 & 4.314117 & -2.602359 & -0.286741\end{array}$

$\begin{array}{lllll}1 & 2.858771 & -1.915181 & -2.185102\end{array}$

$\begin{array}{lllll}1 & 2.798873 & 0.676905 & 2.045838\end{array}$

$\begin{array}{llll}6 & -1.995570 & 0.359502 & 0.436331\end{array}$

$\begin{array}{llll}6 & -3.276564 & -2.071066 & -0.274653\end{array}$

$\begin{array}{llll}6 & -2.258960 & -0.653357 & 1.400773\end{array}$

$\begin{array}{lllll}6 & -2.408117 & 0.102633 & -0.900437\end{array}$

$\begin{array}{lllll}6 & -3.034933 & -1.089614 & -1.242075\end{array}$

$\begin{array}{llll}6 & -2.883495 & -1.840394 & 1.050957\end{array}$

$\begin{array}{llll}1 & -1.955492 & -0.482978 & 2.431182\end{array}$

$\begin{array}{lllll}1 & -2.245508 & 0.855851 & -1.665421\end{array}$

$\begin{array}{lllll}1 & -3.343139 & -1.256763 & -2.271258\end{array}$

$\begin{array}{llll}1 & -3.069616 & -2.595145 & 1.810924\end{array}$

$1-3.767363 \quad-3.001107 \quad-0.547259$

\section{9a)TStransDCB}

UB3LYP/6-31G*

$\begin{array}{llll}6 & 4.419454 & -1.472023 & -0.152966\end{array}$

$\begin{array}{lllll}6 & -1.086285 & 1.052174 & -0.674013\end{array}$

$\begin{array}{lllll}6 & -0.763201 & 2.396341 & -0.076994\end{array}$

$\begin{array}{llll}6 & 1.086285 & 1.052175 & 0.674013\end{array}$

$\begin{array}{lllll}6 & 0.763202 & 2.396341 & 0.076994\end{array}$

$\begin{array}{lllll}1 & 1.223344 & 2.502917 & -0.912911\end{array}$

$\begin{array}{llll}1 & 1.132874 & 3.238402 & 0.684724\end{array}$

$\begin{array}{lllll}1 & -0.482947 & 0.760997 & -1.531667\end{array}$

$\begin{array}{lllll}1 & 0.482948 & 0.760998 & 1.531668\end{array}$

$\begin{array}{lllll}6 & 2.214036 & 0.233064 & 0.380807\end{array}$

$\begin{array}{llll}1 & -1.223344 & 2.502917 & 0.912911\end{array}$

$\begin{array}{lllll}1 & -1.132874 & 3.238401 & -0.684725\end{array}$

$\begin{array}{lllll}1 & 3.081945 & 1.484239 & -1.171045\end{array}$

$\begin{array}{lllll}6 & 3.181020 & 0.560574 & -0.609334\end{array}$

$\begin{array}{llll}6 & 2.403795 & -0.985233 & 1.092647\end{array}$

$\begin{array}{lllll}1 & 3.596594 & -2.741997 & 1.388814\end{array}$

$\begin{array}{llll}6 & 3.480670 & -1.817633 & 0.828509\end{array}$

$\begin{array}{lllll}6 & 4.258999 & -0.277460 & -0.864640\end{array}$

$1 \quad 5.264182-2.123600-0.358056$

$\begin{array}{lllll}1 & 4.984809 & 0.000904 & -1.624780\end{array}$

$\begin{array}{llll}1 & 1.678899 & -1.260701 & 1.855157\end{array}$

$\begin{array}{lllll}6 & -2.214036 & 0.233064 & -0.380807\end{array}$

$\begin{array}{llll}6 & -4.419455 & -1.472022 & 0.152966\end{array}$

$\begin{array}{lllll}6 & -2.403795 & -0.985233 & -1.092648\end{array}$

$\begin{array}{llll}6 & -3.181020 & 0.560574 & 0.609334\end{array}$

$\begin{array}{lllll}6 & -4.258998 & -0.277459 & 0.864641\end{array}$

$\begin{array}{lllll}6 & -3.480671 & -1.817633 & -0.828509\end{array}$

$\begin{array}{lllll}1 & -1.678900 & -1.260701 & -1.855157\end{array}$

$\begin{array}{llll}1 & -3.081943 & 1.484239 & 1.171045\end{array}$

$\begin{array}{llll}1 & -4.984809 & 0.000905 & 1.624781\end{array}$

$1-3.596596 \quad-2.741996-1.388815$

$\begin{array}{llll}1 & -5.264183 & -2.123599 & 0.358056\end{array}$

\section{0a)TStransDCB' \\ UB3LYP/6-31G*}

$\begin{array}{llll}6 & -0.846520 & 0.553625 & 0.937565\end{array}$

$\begin{array}{llll}6 & -0.326320 & 1.958776 & 0.698795\end{array}$

$\begin{array}{lllll}6 & 0.846517 & 0.553621 & -0.937558\end{array}$

$\begin{array}{lllll}6 & 0.326318 & 1.958774 & -0.698794\end{array}$

$\begin{array}{lllll}1 & -0.424622 & 2.187031 & -1.463527\end{array}$

$\begin{array}{llll}1 & 1.110923 & 2.722467 & -0.792449\end{array}$ $\begin{array}{llll}1 & 0.424620 & 2.187036 & 1.463528\end{array}$

$\begin{array}{lllll}1 & -1.110925 & 2.722471 & 0.792448\end{array}$

$\begin{array}{llll}1 & -0.272981 & -0.083472 & 1.604918\end{array}$

$1 \quad 0.272974 \quad-0.083479-1.604906$

$\begin{array}{llll}6 & -2.112072 & 0.063446 & 0.506904\end{array}$

$\begin{array}{lllll}6 & -3.022084 & 0.840527 & -0.262514\end{array}$

$\begin{array}{lllll}6 & -4.248418 & 0.324194 & -0.661164\end{array}$

$\begin{array}{lllll}6 & -4.619673 & -0.980583 & -0.315720\end{array}$

$\begin{array}{llll}6 & -3.741233 & -1.766628 & 0.442585\end{array}$

$\begin{array}{llll}6 & -2.515945 & -1.258675 & 0.846679\end{array}$

$\begin{array}{lllll}1 & -2.756610 & 1.856609 & -0.539184\end{array}$

$\begin{array}{lllll}1 & -4.925210 & 0.942193 & -1.246102\end{array}$

$\begin{array}{lllll}1 & -5.580028 & -1.379152 & -0.630374\end{array}$

$\begin{array}{lllll}1 & -4.021589 & -2.779925 & 0.719265\end{array}$

$\begin{array}{llll}1 & -1.840919 & -1.873811 & 1.437330\end{array}$

$\begin{array}{lllll}6 & 2.112070 & 0.063444 & -0.506901\end{array}$

$\begin{array}{lllll}6 & 3.022087 & 0.840528 & 0.262508\end{array}$

$\begin{array}{lllll}6 & 4.248424 & 0.324197 & 0.661154\end{array}$

$\begin{array}{llll}6 & 4.619676 & -0.980582 & 0.315714\end{array}$

$\begin{array}{lllll}6 & 3.741231 & -1.766630 & -0.442582\end{array}$

$\begin{array}{lllll}6 & 2.515942 & -1.258679 & -0.846671\end{array}$

$\begin{array}{llll}1 & 2.756615 & 1.856612 & 0.539174\end{array}$

$\begin{array}{llll}1 & 4.925219 & 0.942199 & 1.246085\end{array}$

$1 \quad 5.580033 \quad-1.379150 \quad 0.630365$

$14.021586 \quad-2.779929 \quad-0.719258$

$\begin{array}{llll}1 & 1.840912 & -1.873818 & -1.437315\end{array}$

\section{1a)AHx \\ RB3LYP/6-31G*}

$\begin{array}{llll}6 & -3.483805 & -0.626186 & 0.221862\end{array}$

$\begin{array}{llll}6 & -2.375871 & 0.323336 & 0.147008\end{array}$

$\begin{array}{lllll}6 & 0.088216 & 0.683688 & -0.378481\end{array}$

$\begin{array}{lllll}6 & -1.519689 & 2.667189 & -0.241969\end{array}$

$\begin{array}{llll}6 & -0.122277 & 2.133730 & 0.108882\end{array}$

$\begin{array}{llll}6 & -2.598509 & 1.637278 & -0.056867\end{array}$

$\begin{array}{llll}6 & -0.961830 & -0.241372 & 0.298677\end{array}$

$\begin{array}{llll}6 & 1.521542 & 0.225260 & -0.160498\end{array}$

$\begin{array}{llll}1 & -1.740048 & 3.558864 & 0.362690\end{array}$

$\begin{array}{lllll}1 & 0.649466 & 2.784380 & -0.318784\end{array}$

$\begin{array}{llll}6 & -3.286518 & -1.954183 & 0.073325\end{array}$

$\begin{array}{llll}1 & -0.730766 & -0.236767 & 1.381461\end{array}$

$\begin{array}{lllll}1 & -1.849385 & -3.535773 & -0.442711\end{array}$

$\begin{array}{lllll}1 & -0.110137 & 0.658664 & -1.459102\end{array}$

$\begin{array}{lllll}1 & -1.538033 & 3.018923 & -1.286843\end{array}$

$\begin{array}{llll}1 & 0.016041 & 2.162526 & 1.197938\end{array}$

$\begin{array}{llll}1 & -3.629137 & 1.984959 & -0.132831\end{array}$

$\begin{array}{lllll}6 & -0.867862 & -1.690644 & -0.130792\end{array}$

$\begin{array}{lllll}1 & -4.128785 & -2.641013 & 0.092295\end{array}$

$\begin{array}{llll}1 & -4.488606 & -0.223396 & 0.333060\end{array}$

$\begin{array}{lllll}6 & -1.950615 & -2.483097 & -0.188815\end{array}$

$\begin{array}{lllll}1 & 0.124967 & -2.089145 & -0.322243\end{array}$

$\begin{array}{llll}6 & 4.199929 & -0.582441 & 0.213114\end{array}$

$\begin{array}{llll}6 & 2.074420 & 0.129683 & 1.126359\end{array}$

$6 \quad 2.339211 \quad-0.094863-1.252686$

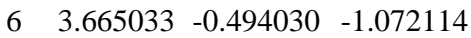

$\begin{array}{lllll}6 & 3.397865 & -0.269454 & 1.312608\end{array}$

$\begin{array}{llll}1 & 1.466452 & 0.369204 & 1.995549\end{array}$

$\begin{array}{lllll}1 & 1.930199 & -0.029113 & -2.258681\end{array}$

$\begin{array}{lllll}1 & 4.277729 & -0.736388 & -1.936722\end{array}$

$\begin{array}{llll}1 & 3.803483 & -0.336045 & 2.318989\end{array}$

$\begin{array}{llll}1 & 5.231118 & -0.893244 & 0.358245\end{array}$ 
22a)AHn

RB3LYP/6-31G*

$\begin{array}{llll}6 & 2.980025 & -0.376762 & 0.847260\end{array}$

$\begin{array}{llll}6 & 1.916912 & 0.520472 & 0.402169\end{array}$

$\begin{array}{llll}6 & -0.157998 & 0.815153 & -1.088521\end{array}$

$\begin{array}{lllll}6 & 0.392846 & 2.493935 & 0.776100\end{array}$

$\begin{array}{lllll}6 & -0.080623 & 2.306196 & -0.673051\end{array}$

$\begin{array}{llll}6 & 1.548433 & 1.596815 & 1.126320\end{array}$

$\begin{array}{llll}6 & 1.252971 & 0.191040 & -0.936424\end{array}$

$\begin{array}{llll}1 & -0.392835 & 0.796689 & -2.162059\end{array}$

$\begin{array}{cccc}1 & 0.666671 & 3.545540 & 0.940792\end{array}$

$\begin{array}{llll}1 & -1.056010 & 2.785398 & -0.816758\end{array}$

$\begin{array}{llll}6 & 3.146302 & -1.602412 & 0.301886\end{array}$

$\begin{array}{lllll}1 & 1.867342 & 0.701197 & -1.703971\end{array}$

$\begin{array}{lllll}1 & 2.332019 & -3.124873 & -1.060783\end{array}$

$\begin{array}{llll}6 & -1.303769 & 0.087069 & -0.390869\end{array}$

$\begin{array}{llll}1 & -0.436815 & 2.296039 & 1.473509\end{array}$

$\begin{array}{lllll}1 & 0.624459 & 2.809598 & -1.348500\end{array}$

$\begin{array}{llll}1 & 2.082485 & 1.813022 & 2.051978\end{array}$

$\begin{array}{lllll}6 & 1.329084 & -1.277844 & -1.293077\end{array}$

$\begin{array}{llll}1 & 3.920992 & -2.267266 & 0.675244\end{array}$

$\begin{array}{llll}1 & 3.598641 & -0.054249 & 1.682543\end{array}$

$\begin{array}{lllll}6 & 2.249347 & -2.087684 & -0.744946\end{array}$

$1 \quad 0.655696 \quad-1.638194 \quad-2.067966$

$\begin{array}{llll}6 & -3.532424 & -1.152797 & 0.824982\end{array}$

$\begin{array}{lllll}6 & -1.153345 & -0.688669 & 0.767333\end{array}$

$\begin{array}{lllll}6 & -2.594952 & 0.221638 & -0.926892\end{array}$

$\begin{array}{lllll}6 & -3.698408 & -0.385704 & -0.329843\end{array}$

$\begin{array}{llll}6 & -2.256450 & -1.302003 & 1.367082\end{array}$

$\begin{array}{llll}1 & -0.170959 & -0.823717 & 1.205553\end{array}$

$\begin{array}{llll}1 & -2.734082 & 0.810426 & -1.831986\end{array}$

$\begin{array}{llll}1 & -4.685201 & -0.266064 & -0.770105\end{array}$

$\begin{array}{llll}1 & -2.112169 & -1.901471 & 2.262487\end{array}$

$\begin{array}{llll}1 & -4.387941 & -1.632733 & 1.292968\end{array}$

23a) $\underline{B H x}$

RB3LYP/6-31G*

$\begin{array}{llll}6 & -3.077674 & 0.348154 & 1.142563\end{array}$

$\begin{array}{llll}6 & -1.835060 & 0.621990 & 0.428379\end{array}$

$\begin{array}{lllll}6 & -0.428185 & 0.089904 & -1.590352\end{array}$

$\begin{array}{llll}6 & -0.007167 & 2.226361 & -0.283605\end{array}$

$\begin{array}{lllll}6 & 0.654394 & 1.077312 & -1.084413\end{array}$

$\begin{array}{llll}6 & -1.220829 & 1.821179 & 0.508913\end{array}$

$\begin{array}{lllll}6 & -1.255344 & -0.496263 & -0.432760\end{array}$

$\begin{array}{lllll}1 & 0.046203 & -0.719333 & -2.159589\end{array}$

$\begin{array}{lllll}1 & 0.737883 & 2.694430 & 0.375192\end{array}$

$\begin{array}{llll}1 & 1.119952 & 1.520529 & -1.974137\end{array}$

$\begin{array}{llll}6 & -3.855149 & -0.709826 & 0.821583\end{array}$

$\begin{array}{lllll}1 & -0.549515 & -1.064695 & 0.203047\end{array}$

$\begin{array}{lllll}1 & -4.192313 & -2.377559 & -0.570635\end{array}$

$\begin{array}{lllll}1 & -1.101039 & 0.619766 & -2.277718\end{array}$

$\begin{array}{lllll}1 & -0.306006 & 3.022060 & -0.984576\end{array}$

$\begin{array}{lllll}6 & 1.764607 & 0.354023 & -0.331184\end{array}$

$\begin{array}{llll}1 & -1.652919 & 2.586935 & 1.154140\end{array}$

$\begin{array}{llll}6 & -2.294914 & -1.496995 & -0.881307\end{array}$

$\begin{array}{llll}1 & -4.796700 & -0.875497 & 1.338888\end{array}$

$\begin{array}{llll}1 & -3.396940 & 1.061178 & 1.900234\end{array}$

$\begin{array}{lllll}6 & -3.483186 & -1.611628 & -0.265653\end{array}$

$\begin{array}{lllll} & 1 & -2.015071 & -2.175655 & -1.685285\end{array}$
$6 \quad 3.838789-1.046096 \quad 0.975980$

$\begin{array}{lllll}6 & 2.850052 & -0.170874 & -1.049199\end{array}$

$\begin{array}{llll}6 & 1.742241 & 0.164287 & 1.058529\end{array}$

$\begin{array}{llll}6 & 2.767898 & -0.529517 & 1.705245\end{array}$

$\begin{array}{lllll}6 & 3.876433 & -0.863435 & -0.407893\end{array}$

$\begin{array}{lllll}1 & 2.892101 & -0.027914 & -2.127454\end{array}$

$\begin{array}{llll}1 & 0.917465 & 0.563280 & 1.642876\end{array}$

$\begin{array}{llll}1 & 2.728143 & -0.662764 & 2.783426\end{array}$

$14.708739 \quad-1.254411 \quad-0.987748$

$14.638586-1.581804 \quad 1.480366$

\section{4a) $\underline{B H n}$}

RB3LYP/6-31G*

$\begin{array}{llll}6 & 3.738261 & 0.985306 & 0.072317\end{array}$

$\begin{array}{lllll}6 & 2.296516 & 0.803785 & -0.070800\end{array}$

$\begin{array}{lllll}6 & 0.360292 & -0.818814 & -0.016502\end{array}$

$\begin{array}{llll}6 & -0.069591 & 1.674056 & 0.144484\end{array}$

$\begin{array}{lllll}6 & -0.555254 & 0.338562 & -0.468148\end{array}$

$\begin{array}{lllll}6 & 1.429390 & 1.808246 & 0.167655\end{array}$

$\begin{array}{lllll}6 & 1.805028 & -0.580336 & -0.489981\end{array}$

$\begin{array}{lllll}1 & -0.014486 & -1.768607 & -0.418338\end{array}$

$\begin{array}{lllll}1 & -0.520101 & 2.512018 & -0.406767\end{array}$

$\begin{array}{llll}6 & -2.025681 & 0.076299 & -0.187873\end{array}$

$\begin{array}{lllll}6 & 4.572411 & -0.072586 & 0.178161\end{array}$

$\begin{array}{lllll}1 & 1.779181 & -0.589897 & -1.597866\end{array}$

$\begin{array}{lllll}1 & 4.736773 & -2.254835 & 0.395061\end{array}$

$\begin{array}{llll}1 & 0.347358 & -0.906843 & 1.077938\end{array}$

$\begin{array}{llll}1 & -0.452270 & 1.773543 & 1.172807\end{array}$

$\begin{array}{lllll}1 & -0.441152 & 0.422249 & -1.558324\end{array}$

$\begin{array}{llll}1 & 1.825799 & 2.787990 & 0.436057\end{array}$

$\begin{array}{lllll}6 & 2.761005 & -1.685268 & -0.097357\end{array}$

$\begin{array}{llll}1 & 5.638436 & 0.080833 & 0.324802\end{array}$

$\begin{array}{lllll}1 & 4.117364 & 2.001438 & 0.161674\end{array}$

$\begin{array}{llll}6 & 4.053682 & -1.439000 & 0.170412\end{array}$

$\begin{array}{lllll}1 & 2.375589 & -2.703329 & -0.107745\end{array}$

$\begin{array}{llll}6 & -4.759061 & -0.415640 & 0.307920\end{array}$

$\begin{array}{llll}6 & -2.495720 & -0.134380 & 1.117758\end{array}$

$\begin{array}{lllll}6 & -2.953598 & 0.035366 & -1.236330\end{array}$

$\begin{array}{lllll}6 & -4.307406 & -0.207347 & -0.995181\end{array}$

$\begin{array}{llll}6 & -3.846391 & -0.378612 & 1.364579\end{array}$

$\begin{array}{llll}1 & -1.799302 & -0.109145 & 1.952656\end{array}$

$\begin{array}{lllll}1 & -2.610297 & 0.195915 & -2.256282\end{array}$

$\begin{array}{llll}1 & -5.007302 & -0.233484 & -1.826563\end{array}$

$\begin{array}{llll}1 & -4.187094 & -0.540485 & 2.384158\end{array}$

$\begin{array}{llll}1 & -5.811785 & -0.605182 & 0.500051\end{array}$

\section{5a)cis-DCB}

RB3LYP/6-31G*

$6 \quad 3.249309-1.687734-0.374253$

$\begin{array}{llll}6 & -0.841998 & 1.552106 & 0.806033\end{array}$

$\begin{array}{llll}6 & -1.184388 & 2.813349 & -0.039012\end{array}$

$\begin{array}{llll}6 & 0.660332 & 1.717775 & 0.288803\end{array}$

$\begin{array}{lllll}6 & 0.113399 & 2.609065 & -0.859111\end{array}$

$\begin{array}{llll}1 & -0.071400 & 2.036242 & -1.773105\end{array}$

$\begin{array}{lllll}1 & 0.703568 & 3.493288 & -1.121467\end{array}$

$\begin{array}{llll}1 & -0.921636 & 1.694233 & 1.889296\end{array}$

$\begin{array}{llll}1 & 1.161423 & 2.366470 & 1.017091\end{array}$

$\begin{array}{llll}6 & 1.551309 & 0.530829 & 0.030172\end{array}$

$\begin{array}{llll}1 & -1.138725 & 3.717911 & 0.575944\end{array}$

$\begin{array}{lllll}1 & -2.136136 & 2.816833 & -0.578308\end{array}$
$1 \quad 0.618385-0.090934-1.815169$

$\begin{array}{lllll}6 & 1.401284 & -0.297305 & -1.092243\end{array}$

$\begin{array}{llll}6 & 2.571194 & 0.221144 & 0.943045\end{array}$

$\begin{array}{llll}1 & 4.195798 & -1.089576 & 1.470143\end{array}$

$\begin{array}{lllll}6 & 3.411553 & -0.874981 & 0.748483\end{array}$

$\begin{array}{lllll}6 & 2.240597 & -1.393395 & -1.293130\end{array}$

$13.903876-2.540658-0.533373$

$\begin{array}{llll}1 & 2.104748 & -2.019555 & -2.171310\end{array}$

$\begin{array}{llll}1 & 2.709539 & 0.853982 & 1.817602\end{array}$

$\begin{array}{llll}6 & -1.543002 & 0.259988 & 0.451194\end{array}$

$\begin{array}{lllll}6 & -2.793551 & -2.208974 & -0.118894\end{array}$

$\begin{array}{llll}6 & -1.515005 & -0.799402 & 1.372112\end{array}$

$\begin{array}{lllll}6 & -2.214724 & 0.052511 & -0.762393\end{array}$

$\begin{array}{lllll}6 & -2.832835 & -1.167565 & -1.045497\end{array}$

$\begin{array}{llll}6 & -2.129935 & -2.018976 & 1.094897\end{array}$

$\begin{array}{llll}1 & -1.002473 & -0.661034 & 2.321592\end{array}$

$\begin{array}{lllll}1 & -2.266124 & 0.849072 & -1.498922\end{array}$

$\begin{array}{llll}1 & -3.350107 & -1.299798 & -1.992562\end{array}$

$1 \quad-2.094182 \quad-2.820068 \quad 1.828811$

$1-3.277792 \quad-3.157003 \quad-0.337510$

\section{6a)trans- $D C B$ \\ RB3LYP/6-31G*}

$\begin{array}{llll}6 & 0.845832 & -3.869105 & -1.549874\end{array}$

$\begin{array}{llll}6 & 0.388764 & 0.684292 & 1.107999\end{array}$

$\begin{array}{llll}6 & 0.130226 & 0.763434 & 2.642932\end{array}$

$\begin{array}{llll}6 & -0.388764 & -0.684292 & 1.107999\end{array}$

$\begin{array}{llll}6 & -0.130226 & -0.763434 & 2.642932\end{array}$

$\begin{array}{lllll}1 & 0.778963 & -1.330487 & 2.868408\end{array}$

$\begin{array}{llll}1 & -0.943372 & -1.150556 & 3.265069\end{array}$

$\begin{array}{llll}6 & -0.047836 & 1.795961 & 0.189637\end{array}$

$\begin{array}{lllll}1 & -1.451644 & -0.476325 & 0.937009\end{array}$

$\begin{array}{lllll}6 & 0.047836 & -1.795961 & 0.189637\end{array}$

$\begin{array}{llll}1 & -0.778963 & 1.330487 & 2.868408\end{array}$

$\begin{array}{llll}1 & 0.943372 & 1.150556 & 3.265069\end{array}$

$\begin{array}{lllll}1 & 2.069614 & -1.909227 & 0.943721\end{array}$

$\begin{array}{llll}6 & 1.352875 & -2.311376 & 0.231144\end{array}$

$\begin{array}{lllll}6 & -0.845832 & -2.341778 & -0.741935\end{array}$

$\begin{array}{lllll}1 & -1.167149 & -3.773373 & -2.317852\end{array}$

$\begin{array}{lllll}6 & -0.454635 & -3.367485 & -1.604200\end{array}$

$\begin{array}{lllll}6 & 1.749120 & -3.335865 & -0.627267\end{array}$

$\begin{array}{lllll}1 & 1.154133 & -4.668193 & -2.218816\end{array}$

$\begin{array}{lllll}1 & 2.765057 & -3.719447 & -0.575949\end{array}$

$\begin{array}{llll}1 & -1.861447 & -1.955335 & -0.792221\end{array}$

$\begin{array}{llll}1 & 1.451644 & 0.476325 & 0.937009\end{array}$

$\begin{array}{llll}6 & -0.845832 & 3.869105 & -1.549874\end{array}$

$\begin{array}{llll}6 & -1.352875 & 2.311376 & 0.231144\end{array}$

$\begin{array}{lllll}6 & 0.845832 & 2.341778 & -0.741935\end{array}$

$\begin{array}{lllll}6 & 0.454635 & 3.367485 & -1.604200\end{array}$

$\begin{array}{lllll}6 & -1.749120 & 3.335865 & -0.627267\end{array}$

$\begin{array}{lllll}1 & -2.069614 & 1.909227 & 0.943721\end{array}$

$\begin{array}{llll}1 & 1.861447 & 1.955335 & -0.792221\end{array}$

$\begin{array}{lllll}1 & 1.167149 & 3.773373 & -2.317852\end{array}$

$\begin{array}{lllll}1 & -2.765057 & 3.719447 & -0.575949\end{array}$

$\begin{array}{lllll}1 & -1.154133 & 4.668193 & -2.218816\end{array}$

27a)AHx + M: TS Habs

UB3LYP/6-31G*

$\begin{array}{llll}6 & 1.369176 & 1.949898 & 1.169085\end{array}$

$\begin{array}{llll}6 & 0.102910 & 2.031192 & 0.456109\end{array}$ 
$\begin{array}{llll}6 & -2.234691 & 1.099791 & 0.064764\end{array}$

$\begin{array}{lllll}6 & -1.520174 & 3.280579 & -1.025189\end{array}$

$\begin{array}{llll}6 & -2.219589 & 1.929740 & -1.236154\end{array}$

$\begin{array}{lllll}6 & -0.230293 & 3.135160 & -0.269234\end{array}$

$\begin{array}{lllll}6 & -0.790837 & 0.847888 & 0.541801\end{array}$

$\begin{array}{lllll}6 & -3.105841 & -0.146734 & -0.019572\end{array}$

$\begin{array}{lllll}1 & -1.336079 & 3.759677 & -1.997516\end{array}$

$\begin{array}{lllll}1 & -3.245608 & 2.082514 & -1.590350\end{array}$

$\begin{array}{llll}6 & 1.657040 & 0.907073 & 1.989966\end{array}$

$\begin{array}{llll}1 & -0.132645 & -0.023212 & -0.502719\end{array}$

$\begin{array}{lllll}1 & 0.922735 & -0.933941 & 2.916089\end{array}$

$\begin{array}{llll}1 & -2.692134 & 1.742341 & 0.835226\end{array}$

$\begin{array}{lllll}1 & -2.194724 & 3.970079 & -0.488912\end{array}$

$\begin{array}{lllll}1 & -1.691270 & 1.375433 & -2.021983\end{array}$

$\begin{array}{lllll}1 & 0.468555 & 3.969951 & -0.298976\end{array}$

$\begin{array}{llll}6 & -0.522136 & -0.101933 & 1.583661\end{array}$

$\begin{array}{llll}1 & 2.612042 & 0.866650 & 2.506531\end{array}$

$\begin{array}{llll}1 & 2.072241 & 2.771239 & 1.050141\end{array}$

$\begin{array}{llll}6 & 0.697369 & -0.145503 & 2.203460\end{array}$

$\begin{array}{llll}1 & -1.252470 & -0.884599 & 1.769141\end{array}$

$\begin{array}{lllll}6 & -4.749752 & -2.442877 & -0.106561\end{array}$

$\begin{array}{lllll}6 & -3.171795 & -0.945555 & -1.170848\end{array}$

$\begin{array}{llll}6 & -3.892014 & -0.519535 & 1.081070\end{array}$

$\begin{array}{llll}6 & -4.702487 & -1.654985 & 1.044140\end{array}$

$\begin{array}{lllll}6 & -3.983165 & -2.080830 & -1.214983\end{array}$

$\begin{array}{lllll}1 & -2.596130 & -0.675669 & -2.050968\end{array}$

$\begin{array}{llll}1 & -3.868436 & 0.093177 & 1.979836\end{array}$

$\begin{array}{llll}1 & -5.301236 & -1.918716 & 1.912217\end{array}$

$\begin{array}{lllll}1 & -4.018761 & -2.680575 & -2.120979\end{array}$

$1-5.382282-3.325841-0.141864$

$1-0.348854-1.366056-1.711914$

$\begin{array}{lllll}1 & 0.828184 & -0.027773 & -2.076206\end{array}$

$\begin{array}{llll}1 & 1.209868 & -2.482576 & -0.263860\end{array}$

$6 \quad 2.888473-1.171848-0.545505$

$\begin{array}{lllll}6 & 0.502160 & -0.782575 & -1.356067\end{array}$

$6 \quad 1.500469-1.527649-0.697518$

$\begin{array}{llll}6 & 5.642784 & -0.582628 & -0.251686\end{array}$

$\begin{array}{llll}6 & 3.425629 & 0.060982 & -0.987724\end{array}$

$\begin{array}{llll}6 & 3.779838 & -2.091922 & 0.057844\end{array}$

$\begin{array}{lllll}6 & 5.131288 & -1.804800 & 0.199424\end{array}$

$\begin{array}{llll}6 & 4.779962 & 0.344579 & -0.843222\end{array}$

$\begin{array}{lllll}1 & 2.772566 & 0.798421 & -1.443270\end{array}$

$\begin{array}{llll}1 & 3.389492 & -3.044047 & 0.410810\end{array}$

$1 \quad 5.791883-2.533642 \quad 0.662324$

$\begin{array}{lllll}1 & 5.167317 & 1.298342 & -1.193203\end{array}$

$1 \quad 6.699816 \quad-0.356222 \quad-0.141983$

\section{8a)AHn + M: TS Habs \\ UB3LYP/6-31G*}

$\begin{array}{llll}6 & -0.759039 & 0.662637 & -1.791689\end{array}$

$\begin{array}{lllll}6 & 0.264894 & 1.004071 & -0.816314\end{array}$

$\begin{array}{llll}6 & 1.898583 & 0.186915 & 0.996069\end{array}$

$\begin{array}{llll}6 & 1.882243 & 2.635370 & 0.226276\end{array}$

$\begin{array}{llll}6 & 1.933112 & 1.676910 & 1.424955\end{array}$

$\begin{array}{lllll}6 & 0.797696 & 2.258816 & -0.742955\end{array}$

$\begin{array}{llll}6 & 0.675053 & -0.071571 & 0.114438\end{array}$

$\begin{array}{llll}1 & 1.775771 & -0.407315 & 1.912990\end{array}$

$\begin{array}{llll}1 & 1.732381 & 3.663881 & 0.582384\end{array}$

$\begin{array}{llll}1 & 2.828941 & 1.865852 & 2.027409\end{array}$

$\begin{array}{llll}6 & -1.182017 & -0.618647 & -1.959992\end{array}$

$\begin{array}{llll}1 & -0.433722 & 0.027650 & 1.096671\end{array}$
$1 \quad-0.908948 \quad-2.715489-1.394591$

$\begin{array}{llll}6 & 3.228952 & -0.251191 & 0.380540\end{array}$

$\begin{array}{lllll}1 & 2.857894 & 2.643608 & -0.287471\end{array}$

$\begin{array}{llll}1 & 1.065671 & 1.869689 & 2.069517\end{array}$

$\begin{array}{lllll}1 & 0.444004 & 3.020388 & -1.436379\end{array}$

$\begin{array}{lllll}6 & 0.352633 & -1.424560 & -0.255222\end{array}$

$\begin{array}{lllll}1 & -1.945135 & -0.847583 & -2.698849\end{array}$

$\begin{array}{lllll}1 & -1.151584 & 1.460563 & -2.418229\end{array}$

$\begin{array}{lllll}6 & -0.607314 & -1.692151 & -1.190007\end{array}$

$\begin{array}{llll}1 & 0.803019 & -2.233936 & 0.316181\end{array}$

$\begin{array}{llll}6 & -4.908133 & 1.107509 & -0.536174\end{array}$

$\begin{array}{llll}1 & -5.016685 & 2.077439 & -1.015308\end{array}$

$1-6.797448 \quad 0.358379 \quad-1.270173$

$\begin{array}{lllll}6 & -5.909617 & 0.142707 & -0.682123\end{array}$

$\begin{array}{llll}1 & -4.507438 & -2.349358 & 1.161113\end{array}$

$\begin{array}{lllll}1 & -6.526955 & -1.863189 & -0.173384\end{array}$

$\begin{array}{llll}6 & -3.769159 & 0.838911 & 0.215436\end{array}$

$\begin{array}{llll}6 & -4.619857 & -1.377845 & 0.684681\end{array}$

$\begin{array}{llll}1 & -3.000805 & 1.599876 & 0.307992\end{array}$

$\begin{array}{llll}6 & -5.756105 & -1.104236 & -0.065793\end{array}$

$\begin{array}{llll}1 & -0.809418 & -0.156827 & 2.880015\end{array}$

$\begin{array}{llll}1 & -1.564815 & 1.201410 & 1.937108\end{array}$

$\begin{array}{llll}1 & -2.408123 & -1.755371 & 2.037212\end{array}$

$\begin{array}{llll}6 & -3.596647 & -0.413395 & 0.853774\end{array}$

$\begin{array}{llll}6 & -1.383931 & 0.124823 & 1.993614\end{array}$

$\begin{array}{llll}6 & -2.449785 & -0.733463 & 1.664152\end{array}$

$\begin{array}{lllll}6 & 5.745508 & -1.009885 & -0.645599\end{array}$

$\begin{array}{lllll}6 & 3.492900 & -0.187796 & -0.994833\end{array}$

$\begin{array}{llll}6 & 4.249588 & -0.707016 & 1.228515\end{array}$

$\begin{array}{llll}6 & 5.495268 & -1.082381 & 0.725886\end{array}$

$\begin{array}{lllll}6 & 4.739132 & -0.562246 & -1.501979\end{array}$

$\begin{array}{lllll}1 & 2.717323 & 0.147545 & -1.676159\end{array}$

$\begin{array}{llll}1 & 4.062050 & -0.771816 & 2.298892\end{array}$

$1 \quad 6.267042 \quad-1.437079 \quad 1.404482$

$\begin{array}{lllll}1 & 4.920080 & -0.507044 & -2.572535\end{array}$

$1 \quad 6.713141-1.304986-1.042829$

\section{9a) $\underline{A \mathrm{Hx}}+\mathrm{M}$ : TS Ene1} RB3LYP/6-31G*

$\begin{array}{llll}1 & -2.34482 & 1.650691 & 1.672138\end{array}$

$\begin{array}{llll}6 & -1.380678 & 1.73464 & 1.177455\end{array}$

$\begin{array}{lllll}6 & 1.151536 & 1.984909 & -0.038374\end{array}$

$\begin{array}{llll}6 & -0.50523 & 0.591446 & 1.199089\end{array}$

$\begin{array}{llll}6 & -0.998322 & 2.912635 & 0.600358\end{array}$

$\begin{array}{lllll}6 & 0.294668 & 3.045205 & -0.003021\end{array}$

$\begin{array}{llll}6 & 0.754975 & 0.66878 & 0.438368\end{array}$

$\begin{array}{llll}6 & -0.904767 & -0.622949 & 1.710246\end{array}$

$\begin{array}{llll}1 & -1.665841 & 3.770392 & 0.623413\end{array}$

$\begin{array}{llll}1 & 0.596021 & 4.006818 & -0.410717\end{array}$

$\begin{array}{lllll}1 & 2.141917 & 2.09521 & -0.470659\end{array}$

$\begin{array}{lllll}6 & -1.614286 & -1.343892 & -0.736473\end{array}$

$\begin{array}{llll}1 & -1.890797 & -0.690775 & 2.166223\end{array}$

$\begin{array}{llll}6 & 0.055415 & -1.746986 & 2.006554\end{array}$

$\begin{array}{llll}6 & 1.334807 & -1.711513 & 1.153679\end{array}$

$\begin{array}{llll}1 & -0.441171 & -2.72014 & 1.893649\end{array}$

$1 \quad 0.328234 \quad-1.688096 \quad 3.074273$

$\begin{array}{lllll}1 & 1.141696 & -2.162844 & 0.175165\end{array}$

$\begin{array}{llll}1 & 2.113298 & -2.324577 & 1.623124\end{array}$

$\begin{array}{llll}6 & 1.871972 & -0.275378 & 0.957694\end{array}$

$1-1.486534-2.341885-0.324511$

$\begin{array}{lllll}6 & -0.566405 & -0.812762 & -1.50078\end{array}$
$1 \quad 0.200325 \quad-1.511375 \quad-1.832343$

$\begin{array}{llll}1 & 0.238131 & 0.01195 & -0.651798\end{array}$

$\begin{array}{llll}1 & -0.794722 & -0.041097 & -2.236714\end{array}$

$\begin{array}{lllll}6 & -2.972421 & -0.815205 & -0.668778\end{array}$

$\begin{array}{lllll}6 & -3.997511 & -1.603772 & -0.101965\end{array}$

$\begin{array}{lllll}6 & -5.311383 & -1.152267 & -0.044677\end{array}$

$\begin{array}{llll}6 & -5.644493 & 0.10944 & -0.548229\end{array}$

$\begin{array}{lllll}6 & -4.644479 & 0.907056 & -1.107197\end{array}$

$\begin{array}{lllll}6 & -3.327121 & 0.455336 & -1.168155\end{array}$

$\begin{array}{llll}1 & -3.748487 & -2.588212 & 0.28923\end{array}$

$1 \quad-6.080167 \quad-1.785435 \quad 0.391182$

$\begin{array}{llll}1 & -6.670651 & 0.464223 & -0.505367\end{array}$

$1-4.890365 \quad 1.890495 \quad-1.500181$

$\begin{array}{lllll}1 & -2.566618 & 1.095739 & -1.602125\end{array}$

$\begin{array}{llll}1 & 2.12805 & 0.106828 & 1.956746\end{array}$

$\begin{array}{llll}6 & 3.163188 & -0.262697 & 0.152385\end{array}$

$\begin{array}{lllll}6 & 3.210463 & -0.639717 & -1.19867\end{array}$

$\begin{array}{llll}6 & 4.415567 & -0.64987 & -1.901452\end{array}$

$\begin{array}{lllll}6 & 5.604494 & -0.283956 & -1.267336\end{array}$

$\begin{array}{lllll}6 & 5.574834 & 0.094958 & 0.074678\end{array}$

$\begin{array}{llll}6 & 4.36554 & 0.105632 & 0.771977\end{array}$

$1 \quad 2.297243 \quad-0.924548-1.711505$

$14.424816 \quad-0.945018-2.947688$

$1 \quad 6.543168 \quad-0.292849 \quad-1.814882$

$\begin{array}{lllll}1 & 6.491845 & 0.384897 & 0.581296\end{array}$

$\begin{array}{llll}1 & 4.353244 & 0.405173 & 1.817791\end{array}$

30a) $\underline{A H n}+\mathrm{M}$ : TS Ene1

RB3LYP/6-31G*

$\begin{array}{llll}1 & 1.505324 & 1.506982 & -1.722002\end{array}$

$\begin{array}{lllll}6 & 0.805763 & 1.58821 & -0.894075\end{array}$

$\begin{array}{llll}6 & -1.061956 & 1.82511 & 1.205402\end{array}$

$\begin{array}{lllll}6 & 0.113946 & 0.395514 & -0.470193\end{array}$

$\begin{array}{lllll}6 & 0.555007 & 2.799854 & -0.31592\end{array}$

$\begin{array}{llll}6 & -0.412161 & 2.927072 & 0.736869\end{array}$

$\begin{array}{llll}6 & -0.731602 & 0.489719 & 0.731681\end{array}$

$\begin{array}{lllll}6 & 0.391985 & -0.843221 & -1.000144\end{array}$

$\begin{array}{lllll}1 & 1.067544 & 3.688535 & -0.675669\end{array}$

$\begin{array}{llll}1 & -0.63538 & 3.911487 & 1.139737\end{array}$

$\begin{array}{llll}1 & -1.814485 & 1.918578 & 1.985714\end{array}$

$\begin{array}{llll}6 & 2.086391 & -1.229086 & 1.082035\end{array}$

$\begin{array}{llll}1 & 1.12528 & -0.910848 & -1.801479\end{array}$

$\begin{array}{llll}6 & -0.505643 & -2.034508 & -0.787131\end{array}$

$\begin{array}{lllll}6 & -1.239962 & -2.00567 & 0.562025\end{array}$

$\begin{array}{lllll}1 & 0.063875 & -2.968943 & -0.875851\end{array}$

$\begin{array}{lllll}1 & -1.242165 & -2.063641 & -1.607753\end{array}$

$\begin{array}{llll}1 & -0.547321 & -2.323107 & 1.348267\end{array}$

$\begin{array}{lllll}1 & -2.061524 & -2.731027 & 0.558954\end{array}$

$\begin{array}{llll}6 & -1.797914 & -0.604815 & 0.932698\end{array}$

$\begin{array}{lllll}1 & 1.869594 & -2.274202 & 0.875098\end{array}$

$\begin{array}{llll}6 & 1.385762 & -0.609358 & 2.12376\end{array}$

$\begin{array}{lllll}1 & 0.846526 & -1.254994 & 2.816801\end{array}$

$\begin{array}{llll}1 & 0.259446 & 0.052797 & 1.563314\end{array}$

$\begin{array}{lllll}1 & -2.037634 & -0.632898 & 2.004745\end{array}$

$\begin{array}{llll}6 & -3.113844 & -0.318239 & 0.2085\end{array}$

$\begin{array}{llll}6 & -4.321067 & -0.664369 & 0.833936\end{array}$

$\begin{array}{llll}6 & -5.54825 & -0.469167 & 0.200025\end{array}$

$\begin{array}{lllll}6 & -5.592408 & 0.082099 & -1.081473\end{array}$

$\begin{array}{llll}6 & -4.400528 & 0.435565 & -1.714949\end{array}$

$\begin{array}{lllll}6 & -3.174307 & 0.239148 & -1.076622\end{array}$

$\begin{array}{lllll} & -4.297882 & -1.091191 & 1.835055\end{array}$ 
$1 \quad-6.468739-0.742191 \quad 0.710012$

$\begin{array}{lllll}1 & -6.546039 & 0.239917 & -1.578315\end{array}$

$\begin{array}{lllll}1 & -4.421878 & 0.872041 & -2.710396\end{array}$

$\begin{array}{lllll}1 & -2.258596 & 0.536074 & -1.57829\end{array}$

$\begin{array}{lllll}1 & 1.822795 & 0.269069 & 2.599575\end{array}$

$\begin{array}{llll}6 & 3.255148 & -0.690777 & 0.395573\end{array}$

$6 \quad 4.011404-1.533326-0.448583$

$\begin{array}{lllll}6 & 5.147902 & -1.072246 & -1.103247\end{array}$

$\begin{array}{lllll}6 & 5.565226 & 0.252726 & -0.940032\end{array}$

$\begin{array}{lllll}6 & 4.829144 & 1.103211 & -0.112944\end{array}$

$\begin{array}{llll}6 & 3.691061 & 0.642441 & 0.546868\end{array}$

$\begin{array}{lllll}1 & 3.694913 & -2.565967 & -0.580891\end{array}$

$\begin{array}{llll}1 & 5.712527 & -1.74671 & -1.742157\end{array}$

$\begin{array}{lllll}1 & 6.453146 & 0.615492 & -1.450591\end{array}$

$\begin{array}{llll}1 & 5.142872 & 2.135504 & 0.021203\end{array}$

$\begin{array}{llll}1 & 3.133227 & 1.323922 & 1.180577\end{array}$

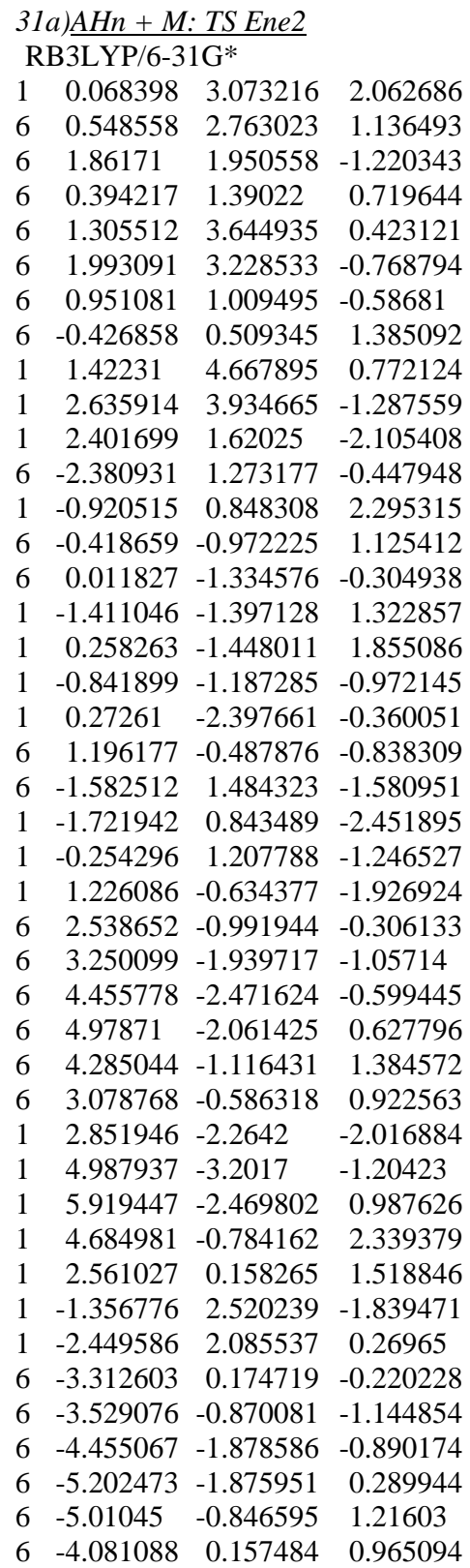

$1-2.980538 \quad-0.884297 \quad-2.082095$

$\begin{array}{lllll}1 & -4.600313 & -2.668173 & -1.623191\end{array}$

$\begin{array}{llll}1 & -5.926872 & -2.662119 & 0.483616\end{array}$

$\begin{array}{llll}1 & -5.587387 & -0.827548 & 2.137179\end{array}$

$\begin{array}{llll}1 & -3.93649 & 0.952036 & 1.693866\end{array}$

32a) $\underline{A H n}+$ M: TS Ene3, b-regio RB3LYP/6-31G*

$\begin{array}{llll}1 & 0.35731 & -1.005997 & 3.195071\end{array}$

$\begin{array}{lllll}6 & 0.224242 & -1.144548 & 2.123311\end{array}$

$\begin{array}{llll}6 & -0.155487 & -1.542754 & -0.648727\end{array}$

$\begin{array}{llll}6 & -0.148031 & -0.019107 & 1.339287\end{array}$

$\begin{array}{llll}6 & 0.349312 & -2.394103 & 1.56826\end{array}$

$\begin{array}{llll}6 & 0.152891 & -2.588089 & 0.161033\end{array}$

$\begin{array}{llll}6 & -0.250291 & -0.15413 & -0.139235\end{array}$

$\begin{array}{llll}6 & -0.175153 & 1.296871 & 1.836213\end{array}$

$\begin{array}{llll}1 & 0.586177 & -3.248791 & 2.196011\end{array}$

$1 \quad 0.239636 \quad-3.590506 \quad-0.250557$

$\begin{array}{llll}1 & -0.323135 & -1.700816 & -1.711916\end{array}$

$\begin{array}{llll}6 & 1.622833 & 2.052683 & 1.238765\end{array}$

$\begin{array}{llll}1 & -0.054248 & 1.399177 & 2.916699\end{array}$

$\begin{array}{llll}6 & -1.190988 & 2.265742 & 1.239297\end{array}$

$\begin{array}{llll}6 & -1.29243 & 2.165951 & -0.288033\end{array}$

$\begin{array}{llll}1 & -0.956897 & 3.29856 & 1.527523\end{array}$

$\begin{array}{llll}1 & -2.169435 & 2.040391 & 1.689601\end{array}$

$\begin{array}{lllll}1 & -0.415558 & 2.644906 & -0.736354\end{array}$

$\begin{array}{lllll}1 & -2.167918 & 2.72284 & -0.641885\end{array}$

$\begin{array}{lllll}6 & -1.367386 & 0.705901 & -0.807994\end{array}$

$\begin{array}{llll}1 & 2.192842 & 1.682216 & 2.087589\end{array}$

$\begin{array}{llll}1 & 1.286072 & 3.078815 & 1.367458\end{array}$

$\begin{array}{llll}6 & 2.073095 & 1.702638 & -0.04798\end{array}$

$\begin{array}{llll}6 & 3.118079 & 0.727752 & -0.350785\end{array}$

$\begin{array}{llll}1 & 1.810529 & 2.36433 & -0.871338\end{array}$

$\begin{array}{llll}1 & 0.7207 & 0.42061 & -0.456539\end{array}$

$\begin{array}{llll}6 & 5.164549 & -1.113243 & -1.024447\end{array}$

$\begin{array}{lllll}6 & 3.601632 & -0.205464 & 0.590643\end{array}$

$\begin{array}{lllll}6 & 3.688385 & 0.700525 & -1.642537\end{array}$

$\begin{array}{lllll}6 & 4.696022 & -0.200078 & -1.972881\end{array}$

$\begin{array}{lllll}6 & 4.606325 & -1.109149 & 0.256586\end{array}$

$\begin{array}{llll}1 & 3.174895 & -0.233934 & 1.588552\end{array}$

$1 \quad 3.333536 \quad 1.408253 \quad-2.389141$

$1 \quad 5.118589-0.189734-2.974686$

$\begin{array}{llll}1 & 4.956356 & -1.81794 & 1.003207\end{array}$

$\begin{array}{llll}1 & 5.950727 & -1.81832 & -1.280082\end{array}$

$\begin{array}{llll}1 & -1.138329 & 0.740313 & -1.881796\end{array}$

$\begin{array}{lllll}6 & -2.773886 & 0.127287 & -0.685424\end{array}$

$\begin{array}{llll}6 & -3.65227 & 0.256951 & -1.772359\end{array}$

$\begin{array}{llll}6 & -4.966754 & -0.20419 & -1.703344\end{array}$

$\begin{array}{lllll}6 & -5.433062 & -0.812501 & -0.536881\end{array}$

$\begin{array}{lllll}6 & -4.570825 & -0.955204 & 0.550507\end{array}$

$\begin{array}{llll}6 & -3.255849 & -0.491179 & 0.477077\end{array}$

$\begin{array}{lllll}1 & -3.297769 & 0.725541 & -2.688635\end{array}$

$\begin{array}{lllll}1 & -5.623573 & -0.093291 & -2.562499\end{array}$

$\begin{array}{llll}1 & -6.455285 & -1.177017 & -0.478671\end{array}$

$\begin{array}{llll}1 & -4.918896 & -1.434673 & 1.462041\end{array}$

$\begin{array}{llll}1 & -2.596046 & -0.626303 & 1.328236\end{array}$

33a) $\underline{A} \cdot$

UB3LYP/6-31G*

$\begin{array}{llll}6 & -3.403839 & -0.683007 & 0.174328\end{array}$ $\begin{array}{llll}6 & -2.352766 & 0.270291 & 0.035348\end{array}$

$\begin{array}{lllll}6 & 0.096507 & 0.798667 & -0.459653\end{array}$

$\begin{array}{lllll}6 & -1.539612 & 2.684254 & 0.050831\end{array}$

$\begin{array}{llll}6 & -0.160230 & 2.086624 & 0.358722\end{array}$

$\begin{array}{lllll}6 & -2.615981 & 1.650200 & 0.155470\end{array}$

$\begin{array}{lllll}6 & -1.029241 & -0.213820 & -0.245510\end{array}$

$\begin{array}{lllll}6 & 1.493959 & 0.260393 & -0.187583\end{array}$

$\begin{array}{llll}1 & -1.749505 & 3.523829 & 0.727733\end{array}$

$\begin{array}{llll}1 & 0.631236 & 2.814433 & 0.147166\end{array}$

$\begin{array}{llll}6 & -3.170648 & -2.040046 & 0.036131\end{array}$

$\begin{array}{lllll}1 & -1.685322 & -3.563091 & -0.361834\end{array}$

$\begin{array}{lllll}1 & 0.066565 & 1.091493 & -1.521970\end{array}$

$\begin{array}{lllll}1 & -1.530426 & 3.123250 & -0.963478\end{array}$

$\begin{array}{llll}1 & -0.098935 & 1.848880 & 1.428318\end{array}$

$\begin{array}{llll}1 & -3.641975 & 1.973319 & 0.316746\end{array}$

$\begin{array}{llll}6 & -0.826827 & -1.583540 & -0.385668\end{array}$

$\begin{array}{lllll}1 & -3.989674 & -2.746325 & 0.145538\end{array}$

$\begin{array}{llll}1 & -4.405521 & -0.319045 & 0.391763\end{array}$

$\begin{array}{lllll}6 & -1.877261 & -2.499814 & -0.248046\end{array}$

$\begin{array}{lllll}1 & 0.171317 & -1.950724 & -0.605289\end{array}$

$\begin{array}{llll}6 & 4.116391 & -0.634671 & 0.331205\end{array}$

$\begin{array}{llll}6 & 1.846886 & -0.241354 & 1.074995\end{array}$

$\begin{array}{lllll}6 & 2.478101 & 0.299809 & -1.182390\end{array}$

$\begin{array}{lllll}6 & 3.778790 & -0.142474 & -0.929046\end{array}$

$\begin{array}{llll}6 & 3.143486 & -0.683614 & 1.333041\end{array}$

$\begin{array}{llll}1 & 1.095097 & -0.295500 & 1.858362\end{array}$

$\begin{array}{lllll}1 & 2.223415 & 0.683469 & -2.168201\end{array}$

$14.525376-0.102352-1.718123$

$\begin{array}{llll}1 & 3.395043 & -1.068971 & 2.317911\end{array}$

$\begin{array}{llll}1 & 5.127013 & -0.980057 & 0.532293\end{array}$

34a)phenylethyl rad (HM•)

UB3LYP/6-31G*

$\begin{array}{llll}6 & 0.461567 & -0.295726 & -0.000002\end{array}$

$\begin{array}{llll}6 & -2.302108 & 0.349591 & 0.000000\end{array}$

$\begin{array}{llll}6 & 0.015499 & 1.057710 & -0.000001\end{array}$

$\begin{array}{llll}6 & -0.541152 & -1.309329 & 0.000000\end{array}$

$\begin{array}{llll}6 & -1.889783 & -0.990799 & 0.000000\end{array}$

$\begin{array}{llll}6 & -1.337772 & 1.365778 & 0.000000\end{array}$

$\begin{array}{llll}1 & 0.747620 & 1.859964 & 0.000002\end{array}$

$\begin{array}{llll}1 & -0.228248 & -2.351188 & 0.000000\end{array}$

$\begin{array}{lllll}1 & -2.631359 & -1.785891 & 0.000001\end{array}$

$\begin{array}{lllll}1 & -1.650586 & 2.407198 & 0.000002\end{array}$

$\begin{array}{llll}1 & -3.359819 & 0.597766 & 0.000001\end{array}$

$\begin{array}{lllll}6 & 1.832873 & -0.646814 & -0.000002\end{array}$

$\begin{array}{llll}1 & 2.076308 & -1.706771 & 0.000013\end{array}$

$\begin{array}{llll}6 & 2.965745 & 0.332481 & 0.000000\end{array}$

$\begin{array}{llll}1 & 2.942951 & 0.991882 & -0.880732\end{array}$

$\begin{array}{llll}1 & 2.943052 & 0.991761 & 0.880827\end{array}$

$\begin{array}{lllll}1 & 3.930871 & -0.182080 & -0.000089\end{array}$

35a)・M2•+ M: TS Habs

UB3LYP/6-31G*

$\begin{array}{llll}1 & 2.916894 & 5.028884 & -0.788794\end{array}$

$\begin{array}{lllll}6 & 2.581478 & 4.098197 & -0.338056\end{array}$

$\begin{array}{llll}6 & 1.712801 & 1.704967 & 0.811317\end{array}$

$\begin{array}{lllll}6 & 1.536401 & 3.389894 & -0.918194\end{array}$

$\begin{array}{llll}6 & 3.202400 & 3.616365 & 0.819040\end{array}$

$\begin{array}{llll}6 & 2.760990 & 2.414190 & 1.383949\end{array}$

$\begin{array}{lllll}6 & 1.066082 & 2.175278 & -0.359202\end{array}$ 
$\begin{array}{llll}1 & 1.058909 & 3.770945 & -1.818371\end{array}$

$\begin{array}{llll}1 & 4.021852 & 4.167079 & 1.272482\end{array}$

$\begin{array}{llll}1 & 3.243903 & 2.025354 & 2.276757\end{array}$

$\begin{array}{llll}6 & -0.033192 & 1.490920 & -0.988558\end{array}$

$\begin{array}{llll}1 & 1.400952 & 0.767411 & 1.260933\end{array}$

$\begin{array}{llll}6 & -0.624360 & 0.274416 & -0.591272\end{array}$

$\begin{array}{lllll}1 & -0.386400 & 1.929078 & -1.922927\end{array}$

$\begin{array}{lllll}1 & 1.636990 & -2.603400 & 0.938315\end{array}$

$6 \quad 0.716982-1.928795 \quad-1.577198$

$\begin{array}{lllll}6 & -2.025708 & -0.065872 & -1.109278\end{array}$

$\begin{array}{llll}1 & -0.479633 & -0.008858 & 0.455797\end{array}$

$\begin{array}{llll}1 & -2.161080 & -1.154580 & -1.121742\end{array}$

$\begin{array}{lllll}1 & -2.088074 & 0.256656 & -2.163425\end{array}$

$1 \quad 2.522978 \quad-1.354179 \quad-2.565986$

$1 \quad 6.420347-1.660911-0.001808$

$1 \quad 5.720606-2.446206 \quad 2.257232$

$14.738604 \quad-1.345108-1.781419$

$\begin{array}{lllll}1 & 0.135425 & -0.744165 & -1.130195\end{array}$

$\begin{array}{llll}6 & 4.982367 & -2.308505 & 1.472111\end{array}$

$\begin{array}{llll}6 & 5.373344 & -1.868814 & 0.203400\end{array}$

$\begin{array}{llll}1 & 3.316001 & -2.908687 & 2.705030\end{array}$

$\begin{array}{llll}6 & 3.629923 & -2.568076 & 1.721445\end{array}$

$\begin{array}{lllll}6 & 2.680680 & -2.394943 & 0.721119\end{array}$

$\begin{array}{lllll}6 & 4.426934 & -1.691396 & -0.798634\end{array}$

$\begin{array}{lllll}1 & 0.189780 & -1.999865 & -2.529999\end{array}$

$\begin{array}{lllll}6 & 2.110302 & -1.760950 & -1.644047\end{array}$

$\begin{array}{llll}6 & 3.054034 & -1.952678 & -0.572009\end{array}$

$\begin{array}{lllll}6 & -3.100397 & 0.588196 & -0.294112\end{array}$

$\begin{array}{llll}6 & -4.361817 & 0.041334 & 0.044691\end{array}$

$\begin{array}{llll}6 & -5.262067 & 0.800882 & 0.851001\end{array}$

$\begin{array}{llll}6 & -6.505473 & 0.307379 & 1.210787\end{array}$

$\begin{array}{llll}6 & -6.914190 & -0.964718 & 0.785303\end{array}$

$\begin{array}{lllll}6 & -6.052653 & -1.731595 & -0.009961\end{array}$

$\begin{array}{llll}6 & -4.805290 & -1.246815 & -0.376924\end{array}$

$\begin{array}{llll}1 & -4.951763 & 1.787782 & 1.186248\end{array}$

$\begin{array}{llll}1 & -7.166639 & 0.911449 & 1.827064\end{array}$

$\begin{array}{lllll}1 & -7.889304 & -1.351183 & 1.067783\end{array}$

$\begin{array}{lllll}1 & -6.363429 & -2.717434 & -0.347095\end{array}$

$\begin{array}{llll}1 & -4.161078 & -1.859062 & -1.000470\end{array}$

$\begin{array}{llll}1 & -2.883179 & 1.593865 & 0.061027\end{array}$

$\begin{array}{lllll}1 & 0.309893 & -2.598014 & -0.816734\end{array}$

36a)M2•

UB3LYP/6-31G*

$\begin{array}{llll}1 & -6.356410 & -0.902689 & 0.523222\end{array}$

$\begin{array}{llll}6 & -5.454896 & -0.336361 & 0.304150\end{array}$

$\begin{array}{lllll}6 & -3.129210 & 1.098532 & -0.250608\end{array}$

$\begin{array}{llll}6 & -4.222324 & -0.986359 & 0.264316\end{array}$

$\begin{array}{llll}6 & -5.529605 & 1.035968 & 0.064786\end{array}$

$\begin{array}{llll}6 & -4.358908 & 1.748915 & -0.211406\end{array}$

$\begin{array}{llll}6 & -3.035687 & -0.286873 & -0.018216\end{array}$

$\begin{array}{llll}1 & -4.172101 & -2.056824 & 0.451317\end{array}$

$1 \quad-6.487690 \quad 1.547625 \quad 0.096529$

$\begin{array}{lllll}1 & -4.405169 & 2.819734 & -0.392702\end{array}$

$\begin{array}{llll}6 & -1.766287 & -1.028861 & -0.053436\end{array}$

$\begin{array}{lllll}1 & -2.230782 & 1.673893 & -0.454690\end{array}$

$\begin{array}{lllll}6 & -0.563263 & -0.577596 & -0.440624\end{array}$

$\begin{array}{llll}1 & -1.839624 & -2.070585 & 0.263663\end{array}$

$\begin{array}{lllll}6 & 0.702652 & -1.404303 & -0.421496\end{array}$

$\begin{array}{llll}1 & -0.440877 & 0.452436 & -0.773318\end{array}$

$\begin{array}{llll}1 & 1.129840 & -1.449373 & -1.430908\end{array}$
$1 \quad 0.447338 \quad-2.438423 \quad-0.145090$

$\begin{array}{llll}6 & 1.698613 & -0.850787 & 0.558658\end{array}$

$\begin{array}{llll}6 & 2.964782 & -0.286705 & 0.274650\end{array}$

$\begin{array}{llll}6 & 3.765382 & 0.211725 & 1.347298\end{array}$

$\begin{array}{lllll}6 & 5.015673 & 0.764080 & 1.123249\end{array}$

$\begin{array}{lllll}6 & 5.530927 & 0.849475 & -0.178177\end{array}$

$\begin{array}{lllll}6 & 4.766928 & 0.370808 & -1.250768\end{array}$

$\begin{array}{lllll}6 & 3.513401 & -0.184271 & -1.038173\end{array}$

$\begin{array}{llll}1 & 3.373359 & 0.148266 & 2.359712\end{array}$

$\begin{array}{llll}1 & 5.599208 & 1.133179 & 1.962912\end{array}$

$\begin{array}{lllll}1 & 6.511540 & 1.282655 & -0.353310\end{array}$

$\begin{array}{llll}1 & 5.158132 & 0.434526 & -2.263142\end{array}$

$\begin{array}{lllll}1 & 2.943912 & -0.544562 & -1.889158\end{array}$

$\begin{array}{llll}1 & 1.380258 & -0.854190 & 1.599745\end{array}$

37a)HM•+ M: TS mono

UB3LYP/6-31G*

$1-3.834772 \quad-2.020332-0.540752$

$\begin{array}{lllll}6 & -3.770034 & -0.958773 & -0.311949\end{array}$

$\begin{array}{llll}6 & -3.609934 & 1.755926 & 0.275472\end{array}$

$\begin{array}{llll}6 & -4.895951 & -0.281914 & 0.146665\end{array}$

$\begin{array}{lllll}6 & -2.535507 & -0.297195 & -0.491877\end{array}$

$\begin{array}{llll}6 & -2.484262 & 1.081303 & -0.184786\end{array}$

$\begin{array}{llll}6 & -4.823555 & 1.081251 & 0.444697\end{array}$

$\begin{array}{llll}1 & -5.832718 & -0.818522 & 0.273796\end{array}$

$\begin{array}{llll}6 & -1.389881 & -1.049141 & -0.977616\end{array}$

$\begin{array}{lllll}1 & -1.553714 & 1.627155 & -0.311159\end{array}$

$\begin{array}{llll}1 & -5.700476 & 1.612372 & 0.804420\end{array}$

$\begin{array}{llll}1 & -3.542878 & 2.816628 & 0.504309\end{array}$

$\begin{array}{llll}6 & -0.114514 & -0.576712 & -1.176792\end{array}$

$\begin{array}{llll}1 & -1.566857 & -2.113124 & -1.131483\end{array}$

$1 \quad 0.610886-1.204427 \quad-1.684719$

$\begin{array}{lllll}1 & 0.091117 & 0.487865 & -1.226448\end{array}$

$\begin{array}{lllll}1 & 1.386746 & -2.380331 & 2.104947\end{array}$

$\begin{array}{llll}1 & 1.323240 & -2.839851 & 0.400325\end{array}$

$\begin{array}{llll}1 & -0.166080 & -2.413029 & 1.251471\end{array}$

$\begin{array}{llll}6 & 1.054541 & -0.714365 & 0.770818\end{array}$

$\begin{array}{llll}6 & 2.331608 & -0.141493 & 0.408936\end{array}$

$\begin{array}{lllll}1 & 5.728414 & 1.513732 & -0.638698\end{array}$

$\begin{array}{llll}6 & 0.893059 & -2.160531 & 1.145750\end{array}$

$\begin{array}{llll}6 & 4.786423 & 1.056086 & -0.349681\end{array}$

$\begin{array}{llll}6 & 2.503155 & 1.265473 & 0.420115\end{array}$

$\begin{array}{llll}6 & 3.437758 & -0.926277 & 0.001606\end{array}$

$\begin{array}{lllll}6 & 4.641298 & -0.334548 & -0.368823\end{array}$

$\begin{array}{llll}6 & 3.706313 & 1.852131 & 0.048829\end{array}$

$\begin{array}{llll}1 & 1.671469 & 1.892139 & 0.735839\end{array}$

$1 \quad 3.351047 \quad-2.008574 \quad-0.016789$

$1 \quad 5.475391 \quad-0.962201-0.672809$

$\begin{array}{llll}1 & 3.808528 & 2.934171 & 0.073655\end{array}$

$\begin{array}{lllll}1 & 0.367557 & -0.031003 & 1.265906\end{array}$

38a)•M2•+M: TS dirad UB3LYP/6-31G*

$\begin{array}{llll}1 & 0.473626 & 3.807145 & 1.771801\end{array}$

$\begin{array}{llll}6 & -0.260537 & 3.943458 & 0.980773\end{array}$

$\begin{array}{lllll}6 & -2.134378 & 4.295409 & -1.045982\end{array}$

$\begin{array}{llll}6 & -0.237872 & 5.101635 & 0.209951\end{array}$

$\begin{array}{llll}6 & -1.221426 & 2.931982 & 0.761470\end{array}$

$\begin{array}{lllll}6 & -2.159578 & 3.137988 & -0.275478\end{array}$

$\begin{array}{lllll}6 & -1.174902 & 5.285632 & -0.810168\end{array}$ $\begin{array}{llll}1 & 0.513431 & 5.863001 & 0.403580\end{array}$

$\begin{array}{llll}6 & -1.204395 & 1.741825 & 1.595635\end{array}$

$\begin{array}{lllll}1 & -2.915061 & 2.383767 & -0.476284\end{array}$

$1-1.158880 \quad 6.188384-1.414554$

$\begin{array}{lllll}1 & -2.867861 & 4.428753 & -1.837306\end{array}$

$\begin{array}{llll}6 & -2.037706 & 0.651837 & 1.496383\end{array}$

$\begin{array}{llll}1 & -0.396008 & 1.699382 & 2.324759\end{array}$

$\begin{array}{lllll}1 & -2.018476 & -0.102790 & 2.276502\end{array}$

$\begin{array}{llll}1 & -2.966801 & 0.704476 & 0.937914\end{array}$

$\begin{array}{lllll}1 & 0.087215 & -1.688827 & 1.445227\end{array}$

$\begin{array}{llll}1 & 0.691709 & -0.207578 & 0.720221\end{array}$

$\begin{array}{lllll}6 & -1.212374 & -0.801375 & -0.031818\end{array}$

$\begin{array}{lllll}6 & -2.271513 & -1.784758 & -0.140887\end{array}$

$\begin{array}{lllll}1 & -5.235859 & -4.334047 & -0.441913\end{array}$

$\begin{array}{llll}6 & 0.149218 & -1.133327 & 0.501675\end{array}$

$\begin{array}{lllll}6 & -4.415934 & -3.626268 & -0.357942\end{array}$

$\begin{array}{lllll}6 & -3.349080 & -1.563730 & -1.033774\end{array}$

$\begin{array}{llll}6 & -2.311380 & -2.963188 & 0.643142\end{array}$

$\begin{array}{llll}6 & -3.364546 & -3.865774 & 0.532132\end{array}$

$\begin{array}{lllll}6 & -4.400155 & -2.466338 & -1.140448\end{array}$

$\begin{array}{llll}1 & -3.340993 & -0.668752 & -1.652532\end{array}$

$\begin{array}{llll}1 & -1.508290 & -3.170447 & 1.344373\end{array}$

$\begin{array}{llll}1 & -3.366561 & -4.764370 & 1.144066\end{array}$

$\begin{array}{llll}1 & -5.209480 & -2.270942 & -1.839450\end{array}$

$\begin{array}{llll}1 & -1.217897 & -0.044158 & -0.815134\end{array}$

$\begin{array}{lllll}6 & 1.006394 & -1.974980 & -0.513927\end{array}$

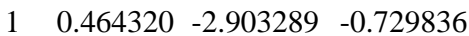

$1 \quad 1.063354-1.418308-1.456946$

$\begin{array}{lllll}6 & 2.364964 & -2.291677 & 0.013678\end{array}$

$1 \quad 2.454786 \quad-3.176520 \quad 0.642441$

$\begin{array}{lllll}6 & 3.526402 & -1.492363 & -0.129547\end{array}$

$\begin{array}{lllll}6 & 3.549984 & -0.271804 & -0.866302\end{array}$

$\begin{array}{lllll}6 & 4.715082 & 0.473116 & -0.982842\end{array}$

$\begin{array}{lllll}6 & 5.903540 & 0.043217 & -0.378392\end{array}$

$\begin{array}{lllll}6 & 5.908535 & -1.153294 & 0.352714\end{array}$

$\begin{array}{lllll}6 & 4.750120 & -1.903197 & 0.477768\end{array}$

$\begin{array}{lllll}1 & 2.641835 & 0.084012 & -1.343176\end{array}$

$\begin{array}{lllll}1 & 4.702133 & 1.400673 & -1.549867\end{array}$

$\begin{array}{lllll}1 & 6.811801 & 0.631290 & -0.474823\end{array}$

$\begin{array}{llll}1 & 6.825285 & -1.495428 & 0.826452\end{array}$

$14.761891 \quad-2.829582 \quad 1.047600$

39a) 2 HM・ TS disp1

UB3LYP/6-31G*

$\begin{array}{llll}1 & -1.058056 & 1.649511 & -1.081303\end{array}$

$\begin{array}{lllll}6 & -1.304393 & 0.591381 & -1.010383\end{array}$

$\begin{array}{lllll}6 & -2.623942 & 0.282015 & -0.540871\end{array}$

$\begin{array}{llll}6 & 1.341609 & -0.783466 & 0.956475\end{array}$

$\begin{array}{llll}6 & -0.280989 & -0.356079 & -1.379067\end{array}$

$\begin{array}{llll}6 & 0.427706 & 0.069338 & 1.777319\end{array}$

$\begin{array}{lllll}6 & 2.634303 & -0.339755 & 0.489994\end{array}$

$\begin{array}{llll}1 & 1.214851 & -1.862132 & 1.034343\end{array}$

$\begin{array}{lllll}6 & 5.159708 & 0.483695 & -0.495643\end{array}$

$\begin{array}{lllll}6 & 3.57359 & -1.276607 & -0.007324\end{array}$

$\begin{array}{llll}6 & 3.004556 & 1.026998 & 0.469038\end{array}$

$\begin{array}{lllll}6 & 4.24627 & 1.426795 & -0.015009\end{array}$

$\begin{array}{llll}6 & 4.812529 & -0.872327 & -0.487857\end{array}$

$\begin{array}{lllll}1 & 3.31315 & -2.332944 & -0.003467\end{array}$

$\begin{array}{llll}1 & 2.307649 & 1.775635 & 0.834884\end{array}$

$\begin{array}{lllll}1 & 4.505521 & 2.482708 & -0.017825\end{array}$

$\begin{array}{lllll}1 & 5.51543 & -1.615255 & -0.856579\end{array}$ 
$\begin{array}{llll}1 & 6.129001 & 0.799884 & -0.870932\end{array}$

$\begin{array}{llll}6 & -5.258619 & -0.240798 & 0.368179\end{array}$

$\begin{array}{lllll}6 & -3.532019 & 1.334306 & -0.253149\end{array}$

$\begin{array}{lllll}6 & -3.088467 & -1.045238 & -0.350205\end{array}$

$\begin{array}{lllll}6 & -4.380338 & -1.295788 & 0.095764\end{array}$

$\begin{array}{llll}6 & -4.822593 & 1.077173 & 0.189086\end{array}$

$\begin{array}{llll}1 & -3.201047 & 2.361703 & -0.38836\end{array}$

$\begin{array}{lllll}1 & -2.424536 & -1.880645 & -0.551781\end{array}$

$\begin{array}{lllll}1 & -4.709023 & -2.322886 & 0.233977\end{array}$

$\begin{array}{llll}1 & -5.495123 & 1.905351 & 0.397874\end{array}$

$\begin{array}{llll}1 & -6.267877 & -0.442985 & 0.715625\end{array}$

$\begin{array}{llll}1 & 0.972715 & 0.734887 & 2.458596\end{array}$

$\begin{array}{llll}1 & -0.258713 & -0.54163 & 2.371116\end{array}$

$\begin{array}{llll}1 & -0.209625 & 0.71846 & 1.139382\end{array}$

$\begin{array}{llll}1 & 0.455932 & 0.047215 & -2.080456\end{array}$

$\begin{array}{lllll}1 & -0.648171 & -1.32567 & -1.729426\end{array}$

$\begin{array}{llll}1 & 0.404461 & -0.641072 & -0.443743\end{array}$

40a)2 HM・ TS disp2

UB3LYP/6-31G*

$\begin{array}{llll}6 & 1.762666 & 1.573629 & 0.831133\end{array}$

$\begin{array}{llll}6 & 2.626134 & 0.514588 & 0.410766\end{array}$

$\begin{array}{llll}6 & -1.543178 & 1.311819 & -0.92664\end{array}$

$\begin{array}{llll}6 & 0.376434 & 1.441997 & 1.204522\end{array}$

$\begin{array}{llll}6 & -1.978732 & 2.751776 & -0.96676\end{array}$

$\begin{array}{lllll}6 & -2.398621 & 0.239292 & -0.474257\end{array}$

$\begin{array}{lllll}1 & -0.799199 & 1.027752 & -1.671815\end{array}$

$\begin{array}{llll}6 & -3.987996 & -1.921234 & 0.444691\end{array}$

$\begin{array}{lllll}6 & -2.036346 & -1.107633 & -0.730711\end{array}$

$\begin{array}{llll}6 & -3.5878 & 0.462079 & 0.261756\end{array}$

$\begin{array}{llll}6 & -4.365228 & -0.601385 & 0.709954\end{array}$

$\begin{array}{lllll}6 & -2.815856 & -2.1655 & -0.280665\end{array}$

$\begin{array}{lllll}1 & -1.128015 & -1.306386 & -1.295832\end{array}$

$\begin{array}{llll}1 & -3.905029 & 1.478438 & 0.475075\end{array}$

$\begin{array}{llll}1 & -5.275863 & -0.400257 & 1.268926\end{array}$

$\begin{array}{lllll}1 & -2.514021 & -3.187145 & -0.497755\end{array}$

$\begin{array}{lllll}1 & -4.598822 & -2.748108 & 0.79608\end{array}$

$\begin{array}{lllll}6 & 4.407589 & -1.530457 & -0.415954\end{array}$

$\begin{array}{llll}6 & 3.970516 & 0.801267 & 0.052906\end{array}$

$\begin{array}{llll}6 & 2.207317 & -0.840754 & 0.330585\end{array}$

$\begin{array}{llll}6 & 3.085048 & -1.838149 & -0.074551\end{array}$

$\begin{array}{lllll}6 & 4.842304 & -0.201305 & -0.348961\end{array}$

$1 \quad 4.315776 \quad 1.831574 \quad 0.103146$

$\begin{array}{llll}1 & 1.183439 & -1.101448 & 0.582092\end{array}$

$1 \quad 2.738312-2.867236-0.128049$

$1 \quad 5.866359 \quad 0.049355-0.614079$

$\begin{array}{lllll}1 & 5.088812 & -2.315286 & -0.732598\end{array}$

$\begin{array}{llll}1 & -2.731117 & 2.927912 & -1.751664\end{array}$

$\begin{array}{lllll}1 & -1.1277 & 3.407751 & -1.179616\end{array}$

$\begin{array}{lllll}1 & -2.421176 & 3.081133 & -0.019214\end{array}$

$\begin{array}{llll}1 & -0.002812 & 2.290391 & 1.782014\end{array}$

$\begin{array}{llll}1 & 0.110061 & 0.500406 & 1.696816\end{array}$

$\begin{array}{llll}1 & -0.358555 & 1.410995 & 0.251112\end{array}$

41a)AHn + HM•: TS tr1a UB3LYP/6-31G*

$\begin{array}{llll}6 & 1.014373 & 3.31071 & -0.301632\end{array}$

$\begin{array}{llll}6 & 1.001074 & 2.107207 & 0.513726\end{array}$

$\begin{array}{lllll}6 & 0.689404 & -0.444622 & 0.592896\end{array}$ $\begin{array}{llll}6 & 1.743721 & 0.83072 & 2.564468\end{array}$

$\begin{array}{llll}6 & 0.772318 & -0.276599 & 2.131503\end{array}$

$\begin{array}{llll}6 & 1.598962 & 2.076607 & 1.738022\end{array}$

$\begin{array}{lllll}6 & 0.304823 & 0.905305 & -0.051479\end{array}$

$\begin{array}{llll}1 & 1.593241 & 1.059001 & 3.628981\end{array}$

$\begin{array}{lllll}1 & 1.056047 & -1.23057 & 2.590754\end{array}$

$\begin{array}{llll}6 & 0.667422 & 3.28319 & -1.615452\end{array}$

$\begin{array}{llll}1 & -0.889011 & 1.022709 & 0.337587\end{array}$

$\begin{array}{lllll}1 & 0.092155 & 2.033102 & -3.320976\end{array}$

$\begin{array}{llll}1 & 2.782967 & 0.470211 & 2.489051\end{array}$

$\begin{array}{llll}1 & -0.231097 & -0.03141 & 2.50097\end{array}$

$\begin{array}{llll}1 & 2.063826 & 2.987404 & 2.114446\end{array}$

$\begin{array}{lllll}6 & 0.151164 & 0.906283 & -1.518678\end{array}$

$\begin{array}{lllll}1 & 0.726615 & 4.187988 & -2.215039\end{array}$

$\begin{array}{llll}1 & 1.383407 & 4.228263 & 0.152512\end{array}$

$\begin{array}{lllll}6 & 0.271799 & 2.044728 & -2.248945\end{array}$

$\begin{array}{lllll}1 & -0.140702 & -0.027675 & -1.993444\end{array}$

$\begin{array}{llll}1 & -2.221139 & 1.176251 & 1.851467\end{array}$

$\begin{array}{llll}6 & -2.397868 & 1.122237 & 0.775792\end{array}$

$\begin{array}{llll}6 & -2.743897 & 2.440019 & 0.126487\end{array}$

$\begin{array}{lllll}1 & -2.553808 & 2.429725 & -0.952259\end{array}$

$\begin{array}{llll}1 & -3.805802 & 2.691463 & 0.271289\end{array}$

$\begin{array}{llll}1 & -2.146418 & 3.250521 & 0.554196\end{array}$

$\begin{array}{llll}6 & -2.981948 & -0.128209 & 0.324698\end{array}$

$\begin{array}{llll}6 & -3.011543 & -1.253858 & 1.185622\end{array}$

$\begin{array}{llll}6 & -3.528063 & -2.474016 & 0.766961\end{array}$

$\begin{array}{lllll}6 & -4.035344 & -2.618853 & -0.529545\end{array}$

$\begin{array}{lllll}6 & -4.018042 & -1.522424 & -1.396204\end{array}$

$\begin{array}{llll}6 & -3.50184 & -0.297863 & -0.98105\end{array}$

$\begin{array}{llll}1 & -2.628411 & -1.149199 & 2.19847\end{array}$

$\begin{array}{llll}1 & -3.543254 & -3.31639 & 1.454139\end{array}$

$\begin{array}{llll}1 & -4.441402 & -3.571832 & -0.857112\end{array}$

$1-4.413255-1.622321-2.40416$

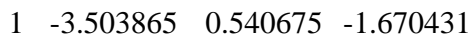

$\begin{array}{llll}1 & -0.138718 & -1.13767 & 0.39547\end{array}$

$\begin{array}{llll}6 & 1.944978 & -1.103936 & 0.025052\end{array}$

$\begin{array}{lllll}6 & 2.002229 & -2.505753 & -0.018057\end{array}$

$\begin{array}{lllll}6 & 3.133691 & -3.172298 & -0.487471\end{array}$

$\begin{array}{lllll}6 & 4.239437 & -2.444396 & -0.930542\end{array}$

$\begin{array}{lllll}6 & 4.19653 & -1.050464 & -0.899239\end{array}$

$\begin{array}{llll}6 & 3.061972 & -0.386594 & -0.426986\end{array}$

$\begin{array}{llll}1 & 1.141975 & -3.081941 & 0.317603\end{array}$

$1 \quad 3.148086-4.259067-0.513858$

$1 \quad 5.122216 \quad-2.958484 \quad-1.301607$

$1 \quad 5.048522-0.471711-1.247367$

$\begin{array}{lllll}1 & 3.045715 & 0.698241 & -0.420365\end{array}$

42a)AHx + HM•: TS tr1a

UB3LYP/6-31G*

$\begin{array}{llll}6 & 0.983834 & 3.103044 & 1.144862\end{array}$

$\begin{array}{llll}6 & -0.173167 & 2.614372 & 0.415492\end{array}$

$\begin{array}{llll}6 & -1.948139 & 0.820204 & 0.154019\end{array}$

$\begin{array}{lllll}6 & -2.227747 & 3.071791 & -0.996378\end{array}$

$\begin{array}{lllll}6 & -2.362893 & 1.548723 & -1.140208\end{array}$

$\begin{array}{lllll}6 & -0.968667 & 3.472976 & -0.283616\end{array}$

$\begin{array}{llll}6 & -0.45633 & 1.14018 & 0.472158\end{array}$

$\begin{array}{llll}6 & -2.304132 & -0.660728 & 0.153578\end{array}$

$\begin{array}{llll}1 & -2.270898 & 3.545968 & -1.987847\end{array}$

$\begin{array}{llll}1 & -3.393378 & 1.28375 & -1.404201\end{array}$

$\begin{array}{llll}6 & 1.634297 & 2.325624 & 2.050504\end{array}$

$\begin{array}{llll}1 & 0.205696 & 0.65553 & -0.507508\end{array}$ $\begin{array}{llll}1 & 1.66708 & 0.409506 & 3.106897\end{array}$

$\begin{array}{llll}1 & -2.523481 & 1.275124 & 0.974565\end{array}$

$\begin{array}{lllll}1 & -3.097611 & 3.474225 & -0.449882\end{array}$

$\begin{array}{lllll}1 & -1.728533 & 1.212455 & -1.969201\end{array}$

$\begin{array}{lllll}1 & -0.704694 & 4.530065 & -0.297392\end{array}$

$\begin{array}{llll}6 & 0.154673 & 0.441748 & 1.615505\end{array}$

$\begin{array}{llll}1 & 2.476605 & 2.724929 & 2.609701\end{array}$

$\begin{array}{llll}1 & 1.277018 & 4.140122 & 0.994137\end{array}$

$\begin{array}{llll}6 & 1.181173 & 0.983225 & 2.322239\end{array}$

$\begin{array}{llll}1 & -0.170731 & -0.573219 & 1.821457\end{array}$

$\begin{array}{llll}6 & -3.010598 & -3.397459 & 0.22632\end{array}$

$\begin{array}{llll}6 & -2.232873 & -1.458158 & -0.998346\end{array}$

$\begin{array}{llll}6 & -2.752683 & -1.265702 & 1.33809\end{array}$

$\begin{array}{lllll}6 & -3.097973 & -2.616811 & 1.379811\end{array}$

$\begin{array}{llll}6 & -2.58056 & -2.810485 & -0.964294\end{array}$

$1 \quad-1.919078-1.020909-1.940995$

$\begin{array}{llll}1 & -2.834457 & -0.66415 & 2.240834\end{array}$

$\begin{array}{llll}1 & -3.442477 & -3.056506 & 2.312321\end{array}$

$1-2.521255 \quad-3.403055-1.873816$

$\begin{array}{llll}1 & -3.283411 & -4.449022 & 0.252655\end{array}$

$\begin{array}{lllll}1 & 0.290083 & -0.436409 & -2.181956\end{array}$

$\begin{array}{lllll}6 & 1.088173 & 0.108415 & -1.676951\end{array}$

$\begin{array}{lllll}6 & 1.528184 & 1.372715 & -2.374613\end{array}$

$\begin{array}{lllll}1 & 2.027126 & 2.068897 & -1.691154\end{array}$

$1 \quad 2.230636 \quad 1.157818$-3.194854

$\begin{array}{lllll}1 & 0.671507 & 1.900219 & -2.805785\end{array}$

$\begin{array}{lllll}6 & 2.042274 & -0.750922 & -0.992889\end{array}$

$\begin{array}{lllll}6 & 1.669931 & -2.065019 & -0.621168\end{array}$

$\begin{array}{llll}6 & 2.55122 & -2.90292 & 0.052943\end{array}$

$\begin{array}{llll}6 & 3.836373 & -2.459344 & 0.381685\end{array}$

$\begin{array}{llll}6 & 4.22327 & -1.163101 & 0.028345\end{array}$

$\begin{array}{lllll}6 & 3.342904 & -0.320313 & -0.643264\end{array}$

$\begin{array}{lllll}1 & 0.67258 & -2.419561 & -0.870096\end{array}$

$\begin{array}{llll}1 & 2.237462 & -3.90871 & 0.3215\end{array}$

$14.526785 \quad-3.114952 \quad 0.905469$

$1 \quad 5.220171 \quad-0.807626 \quad 0.277706$

$\begin{array}{llll}1 & 3.664018 & 0.68317 & -0.905504\end{array}$

43a) cis A-Sty

RB3LYP/6-31G*

$\begin{array}{llll}6 & -0.063909 & 3.170689 & 0.071105\end{array}$ $\begin{array}{llll}6 & -0.157277 & 1.825139 & -0.324099\end{array}$ $6-1.652843-0.139501-1.078539$ $\begin{array}{lllll}6 & 0.823278 & -0.052639 & -1.662188\end{array}$ $\begin{array}{llll}6 & -0.341911 & -0.943628 & -1.225658\end{array}$ $\begin{array}{llll}6 & 1.116849 & 1.035329 & -0.609901\end{array}$ $\begin{array}{llll}6 & -1.437811 & 1.255989 & -0.481066\end{array}$ $\begin{array}{llll}6 & -2.711944 & -0.967361 & -0.360313\end{array}$ $\begin{array}{llll}1 & 1.717105 & -0.657919 & -1.842704\end{array}$ $1-0.504230-1.752841-1.947667$ $\begin{array}{llll}6 & -1.192260 & 3.935921 & 0.348925\end{array}$ $\begin{array}{llll}1-3.351719 & 3.940585 & 0.435626\end{array}$ $\begin{array}{llll}1-2.027382 & 0.033634 & -2.099250\end{array}$ $\begin{array}{lllll}1 & 0.571855 & 0.435551 & -2.614072\end{array}$ $\begin{array}{llll}1 & -0.103708 & -1.433473 & -0.274178\end{array}$ $6-2.568922 \quad 2.038476 \quad-0.196336$ $\begin{array}{llll}1 & -1.085405 & 4.973848 & 0.653266\end{array}$ $\begin{array}{llll}1 & 0.920714 & 3.624342 & 0.154638\end{array}$ $\begin{array}{llll}6 & -2.457736 & 3.360040 & 0.223163\end{array}$ $1-3.554454 \quad 1.595823-0.316031$ $\begin{array}{llll}6 & -4.610520 & -2.599715 & 0.934182\end{array}$ 
$6-2.674816-1.149708 \quad 1.030381$

$6-3.719063-1.614501-1.086423$

$\begin{array}{llll}6 & -4.661265 & -2.424807 & -0.448447\end{array}$

$\begin{array}{llll}6 & -3.612920 & -1.957069 & 1.671969\end{array}$

$\begin{array}{llll}1 & -1.910713 & -0.642715 & 1.614695\end{array}$

$\begin{array}{llll}1 & -3.765917 & -1.482920 & -2.165646\end{array}$

$\begin{array}{llll}1 & -5.435297 & -2.915218 & -1.033334\end{array}$

$\begin{array}{llll}1 & -3.567708 & -2.082607 & 2.750887\end{array}$

$\begin{array}{llll}1 & -5.343133 & -3.227265 & 1.434724\end{array}$

$\begin{array}{llll}1 & 1.847956 & 1.736392 & -1.036116\end{array}$

$\begin{array}{llll}6 & 1.776141 & 0.437587 & 0.686422\end{array}$

$\begin{array}{llll}1 & 1.050264 & -0.252582 & 1.134512\end{array}$

$\begin{array}{llll}6 & 2.099374 & 1.497930 & 1.757157\end{array}$

$\begin{array}{llll}1 & 2.570562 & 1.022670 & 2.624333\end{array}$

$\begin{array}{llll}1 & 2.798149 & 2.253260 & 1.377350\end{array}$

$\begin{array}{llll}1 & 1.197462 & 2.013166 & 2.098352\end{array}$

$\begin{array}{llll}6 & 3.028531 & -0.371172 & 0.365617\end{array}$

$\begin{array}{lllll}6 & 3.104559 & -1.730843 & 0.695750\end{array}$

$\begin{array}{lllll}6 & 4.255764 & -2.476873 & 0.433849\end{array}$

$\begin{array}{lllll}6 & 5.359327 & -1.872608 & -0.167968\end{array}$

$\begin{array}{lllll}6 & 5.300515 & -0.517916 & -0.502841\end{array}$

$\begin{array}{llll}6 & 4.148950 & 0.222579 & -0.236834\end{array}$

$\begin{array}{llll}1 & 2.250577 & -2.212053 & 1.167896\end{array}$

$\begin{array}{lllll}1 & 4.287760 & -3.530121 & 0.701074\end{array}$

$\begin{array}{lllll}1 & 6.256961 & -2.449536 & -0.373917\end{array}$

$\begin{array}{lllll}1 & 6.154845 & -0.035255 & -0.970893\end{array}$

$\begin{array}{lllll}1 & 4.124919 & 1.277204 & -0.500990\end{array}$

44a)trans A-Sty

RB3LYP/6-31G*

$\begin{array}{llll}6 & 0.206763 & 2.845467 & 0.697726\end{array}$

$\begin{array}{llll}6 & 0.253762 & 1.533544 & 0.192161\end{array}$

$\begin{array}{llll}6 & 1.612903 & -0.289553 & -1.028475\end{array}$

$\begin{array}{llll}6 & -0.391737 & -0.884216 & 0.430343\end{array}$

$\begin{array}{lllll}6 & 0.346320 & -1.170210 & -0.879935\end{array}$

$\begin{array}{llll}6 & -0.896396 & 0.571741 & 0.480552\end{array}$

$\begin{array}{llll}6 & 1.381765 & 1.145501 & -0.558144\end{array}$

$\begin{array}{llll}1 & -1.228526 & -1.578395 & 0.552413\end{array}$

$\begin{array}{llll}1 & 0.631635 & -2.226358 & -0.936783\end{array}$

$\begin{array}{llll}6 & 1.206628 & 3.774239 & 0.428009\end{array}$

$\begin{array}{llll}1 & 3.084513 & 4.113917 & -0.588305\end{array}$

$\begin{array}{llll}1 & 0.287883 & -1.059326 & 1.274228\end{array}$

$\begin{array}{llll}1 & -0.321431 & -0.983434 & -1.730192\end{array}$

$\begin{array}{llll}6 & 2.379895 & 2.094873 & -0.832205\end{array}$

$\begin{array}{llll}1 & 1.137918 & 4.780795 & 0.832311\end{array}$

$\begin{array}{llll}1 & -0.635589 & 3.136943 & 1.320066\end{array}$

$\begin{array}{llll}6 & 2.297359 & 3.400033 & -0.360405\end{array}$

$\begin{array}{llll}1 & 3.247627 & 1.786687 & -1.411532\end{array}$

$\begin{array}{llll}1-1.248793 & 0.778969 & 1.500692\end{array}$

$\begin{array}{llll}6 & -2.132381 & 0.785992 & -0.470347\end{array}$

$\begin{array}{llll}1-1.796528 & 0.573753 & -1.493212\end{array}$

$\begin{array}{llll}6 & -2.678067 & 2.227473 & -0.475688\end{array}$

$\begin{array}{llll}1-3.545757 & 2.293978 & -1.141010\end{array}$

$\begin{array}{llll}1-3.007227 & 2.538937 & 0.523173\end{array}$

$\begin{array}{llll}1 & -1.927013 & 2.941579 & -0.823363\end{array}$

$6-3.262363-0.188541-0.154087$

$6-3.719507-1.091644-1.123053$

$\begin{array}{llll}6 & -4.773003 & -1.968794 & -0.856560\end{array}$

$\begin{array}{llll}6 & -5.392396 & -1.959265 & 0.393057\end{array}$

$\begin{array}{llll}6 & -4.949785 & -1.064544 & 1.369875\end{array}$

$\begin{array}{llll}6 & -3.898788 & -0.189290 & 1.097019\end{array}$
$1-3.244485-1.106772-2.101806$

$\begin{array}{llll}1 & -5.107767 & -2.658901 & -1.626941\end{array}$

$1-6.212053 \quad-2.640637 \quad 0.604882$

$\begin{array}{llll}1 & -5.425800 & -1.046260 & 2.347094\end{array}$

$\begin{array}{llll}1 & -3.572326 & 0.503310 & 1.869213\end{array}$

$\begin{array}{lllll}1 & 1.863159 & -0.252427 & -2.097599\end{array}$

$\begin{array}{llll}6 & 2.810991 & -0.943110 & -0.331795\end{array}$

$\begin{array}{lllll}6 & 3.582093 & -1.880801 & -1.034504\end{array}$

$\begin{array}{lllll}6 & 4.645079 & -2.545721 & -0.423532\end{array}$

$\begin{array}{llll}6 & 4.959760 & -2.283304 & 0.911178\end{array}$

$\begin{array}{lllll}6 & 4.205482 & -1.348782 & 1.621228\end{array}$

$\begin{array}{llll}6 & 3.144200 & -0.682856 & 1.004518\end{array}$

$13.344748-2.091275-2.075736$

$\begin{array}{lllll}1 & 5.230598 & -3.264294 & -0.991605\end{array}$

$\begin{array}{lllll}1 & 5.788783 & -2.797421 & 1.390291\end{array}$

$\begin{array}{lllll}1 & 4.445208 & -1.130511 & 2.658905\end{array}$

$\begin{array}{llll}1 & 2.579758 & 0.057749 & 1.563201\end{array}$

45a) $\underline{\text { AH model }}$

RB3LYP/6-31G*

$\begin{array}{llll}6 & -1.386259 & 1.433589 & 0.112255\end{array}$

$\begin{array}{llll}6 & -0.087948 & 0.765792 & 0.121361\end{array}$

$\begin{array}{lllll}6 & 1.171122 & -1.391027 & -0.25572\end{array}$

$\begin{array}{lllll}6 & 2.421528 & 0.821905 & -0.200877\end{array}$

$\begin{array}{llll}6 & 2.437876 & -0.662338 & 0.209333\end{array}$

$\begin{array}{lllll}6 & 1.056417 & 1.447262 & -0.0914\end{array}$

$\begin{array}{llll}6 & -0.078545 & -0.743048 & 0.368623\end{array}$

$\begin{array}{llll}1 & 3.144888 & 1.387154 & 0.40452\end{array}$

$\begin{array}{lllll}1 & 3.334225 & -1.151706 & -0.190615\end{array}$

$\begin{array}{llll}6 & -2.536455 & 0.735958 & -0.01516\end{array}$

$\begin{array}{llll}1 & 0.006101 & -0.888889 & 1.463854\end{array}$

$\begin{array}{llll}1 & -3.449181 & -1.22679 & -0.402769\end{array}$

$\begin{array}{lllll}1 & 1.091577 & -1.346854 & -1.350609\end{array}$

$\begin{array}{lllll}1 & 2.774009 & 0.928326 & -1.240204\end{array}$

$\begin{array}{llll}1 & 2.503773 & -0.735805 & 1.303716\end{array}$

$\begin{array}{lllll}1 & 1.000203 & 2.526469 & -0.237924\end{array}$

$\begin{array}{lllll}6 & -1.375317 & -1.410714 & -0.032317\end{array}$

$\begin{array}{llll}1 & -3.491089 & 1.25385 & -0.059747\end{array}$

$\begin{array}{llll}1 & -1.398603 & 2.521399 & 0.142733\end{array}$

$\begin{array}{lllll}6 & -2.51745 & -0.717459 & -0.167764\end{array}$

$\begin{array}{lllll}1 & -1.362688 & -2.493845 & -0.14233\end{array}$

$\begin{array}{llll}1 & 1.216973 & -2.45283 & 0.019365\end{array}$

46a) AH model + M: TS Habs1a UB3LYP/6-31G*

$\begin{array}{llll}6 & -0.714644 & 1.552105 & 1.157604\end{array}$

$\begin{array}{llll}6 & -1.808688 & 0.940799 & 0.42223\end{array}$

$\begin{array}{llll}6 & -3.262001 & -1.109491 & 0.02122\end{array}$

$\begin{array}{lllll}6 & -3.802117 & 1.087595 & -1.129938\end{array}$

$\begin{array}{lllll}6 & -3.683111 & -0.437036 & -1.294185\end{array}$

$\begin{array}{lllll}6 & -2.63742 & 1.673049 & -0.379384\end{array}$

$\begin{array}{llll}6 & -1.96698 & -0.522523 & 0.563643\end{array}$

$\begin{array}{lllll}1 & -3.155124 & -2.193462 & -0.117881\end{array}$

$\begin{array}{llll}1 & -3.893611 & 1.564691 & -2.115871\end{array}$

$\begin{array}{lllll}1 & -4.633804 & -0.855336 & -1.646089\end{array}$

$\begin{array}{llll}6 & 0.006608 & 0.848457 & 2.072274\end{array}$

$\begin{array}{llll}1 & -0.960464 & -0.987563 & -0.423918\end{array}$

$\begin{array}{lllll}1 & 0.235249 & -1.044958 & 3.144191\end{array}$

$\begin{array}{lllll}1 & -4.741497 & 1.331871 & -0.604277\end{array}$
$1-2.936436-0.657563 \quad-2.068194$

$\begin{array}{llll}1 & -2.470002 & 2.74598 & -0.46321\end{array}$

$\begin{array}{llll}6 & -1.317945 & -1.152235 & 1.68275\end{array}$

$\begin{array}{llll}1 & 0.815771 & 1.329796 & 2.614807\end{array}$

$\begin{array}{lllll}1 & -0.511956 & 2.607507 & 0.98874\end{array}$

$\begin{array}{llll}6 & -0.309099 & -0.526283 & 2.360036\end{array}$

$\begin{array}{llll}1 & -1.565053 & -2.189651 & 1.902326\end{array}$

$\begin{array}{llll}6 & 3.203018 & 1.516736 & -0.822308\end{array}$

$\begin{array}{lllll}1 & 3.101204 & 2.559327 & -1.113541\end{array}$

$1 \quad 5.254608 \quad 1.734238 \quad-0.178688$

$\begin{array}{lllll}6 & 4.413788 & 1.056063 & -0.295648\end{array}$

$\begin{array}{llll}1 & 3.55317 & -2.193613 & 0.226832\end{array}$

$1 \quad 5.466351 \quad-0.656308 \quad 0.494003$

$\begin{array}{lllll}6 & 2.12263 & 0.654651 & -0.976038\end{array}$

$\begin{array}{llll}6 & 3.45462 & -1.151217 & -0.068501\end{array}$

$\begin{array}{llll}1 & 1.189089 & 1.036 & -1.377442\end{array}$

$\begin{array}{llll}6 & 4.530929 & -0.286412 & 0.081585\end{array}$

$\begin{array}{lllll}1 & -0.660819 & -2.279895 & -1.691768\end{array}$

$\begin{array}{llll}1 & -0.201928 & -0.55539 & -2.032614\end{array}$

$\begin{array}{lllll}1 & 1.327453 & -2.648132 & -0.375471\end{array}$

$\begin{array}{lllll}6 & 2.221303 & -0.709433 & -0.607366\end{array}$

$\begin{array}{lllll}6 & -0.119202 & -1.397777 & -1.340786\end{array}$

$\begin{array}{lllll}6 & 1.143012 & -1.649662 & -0.76873\end{array}$

$\begin{array}{llll}1 & -4.060414 & -0.971861 & 0.767313\end{array}$

47a) AH model + M: TS Habs1b

UB3LYP/6-31G*

$\begin{array}{llll}6 & 2.314523 & 2.118443 & 0.156448\end{array}$

$\begin{array}{llll}6 & 2.66616 & 0.716832 & -0.004038\end{array}$

$\begin{array}{lllll}6 & 2.069481 & -1.564289 & -0.966468\end{array}$

$\begin{array}{llll}6 & 4.250275 & -1.231595 & 0.283026\end{array}$

$\begin{array}{llll}6 & 3.025788 & -2.14156 & 0.086567\end{array}$

$\begin{array}{llll}6 & 3.860268 & 0.215376 & 0.419569\end{array}$

$\begin{array}{lllll}6 & 1.630562 & -0.152739 & -0.609712\end{array}$

$\begin{array}{lllll}1 & 1.19233 & -2.212485 & -1.089863\end{array}$

$\begin{array}{llll}1 & 4.817342 & -1.55238 & 1.168129\end{array}$

$\begin{array}{llll}1 & 3.344149 & -3.150511 & -0.20186\end{array}$

$\begin{array}{llll}6 & 1.16634 & 2.625516 & -0.364408\end{array}$

$\begin{array}{lllll}1 & 0.749615 & -0.314875 & 0.584766\end{array}$

$\begin{array}{llll}1 & -0.585751 & 2.248293 & -1.628595\end{array}$

$14.945329 \quad-1.355939-0.565394$

$\begin{array}{llll}1 & 2.493838 & -2.238135 & 1.042566\end{array}$

$\begin{array}{llll}1 & 4.578344 & 0.89225 & 0.880388\end{array}$

$\begin{array}{llll}6 & 0.581589 & 0.48072 & -1.35506\end{array}$

$\begin{array}{lllll}1 & 0.924888 & 3.677127 & -0.229823\end{array}$

$\begin{array}{llll}1 & 3.011915 & 2.76203 & 0.688668\end{array}$

$\begin{array}{llll}6 & 0.27708 & 1.798016 & -1.146854\end{array}$

$\begin{array}{lllll}1 & -0.075924 & -0.143319 & -1.957096\end{array}$

$\begin{array}{lllll}6 & -3.796824 & -1.659993 & -0.403223\end{array}$

$\begin{array}{lllll}1 & -3.921959 & -2.68009 & -0.758048\end{array}$

$1-5.773563-1.036057-1.016335$

$\begin{array}{lllll}6 & -4.838544 & -0.738218 & -0.550138\end{array}$

$\begin{array}{llll}1 & -3.335468 & 1.971778 & 0.854679\end{array}$

$\begin{array}{lllll}1 & -5.463497 & 1.297824 & -0.198764\end{array}$

$\begin{array}{lllll}6 & -2.598367 & -1.284985 & 0.193781\end{array}$

$\begin{array}{llll}6 & -3.464249 & 0.950689 & 0.502331\end{array}$

$\begin{array}{llll}1 & -1.803675 & -2.018676 & 0.293993\end{array}$

$\begin{array}{llll}6 & -4.662177 & 0.571046 & -0.091056\end{array}$

$\begin{array}{llll}1 & -0.22397 & -1.41032 & 1.701776\end{array}$

$\begin{array}{llll}1 & 0.614147 & 0.051634 & 2.377646\end{array}$

$\begin{array}{llll}1 & -1.130519 & 1.522264 & 1.570571\end{array}$ 
$\begin{array}{llll}6 & -2.397565 & 0.034398 & 0.666152\end{array}$

$\begin{array}{llll}6 & -0.061864 & -0.333124 & 1.612331\end{array}$

$\begin{array}{llll}6 & -1.178782 & 0.468159 & 1.308221\end{array}$

$\begin{array}{lllll}1 & 2.58026 & -1.546572 & -1.942216\end{array}$

48a)AH model + M: TS Ene1 RB3LYP/6-31G*

$1-1.563093 \quad 1.918594-2.310303$

$\begin{array}{lllll}6 & -1.330845 & 1.834851 & -1.249793\end{array}$

$\begin{array}{llll}6 & -0.758685 & 1.665554 & 1.513921\end{array}$

$\begin{array}{llll}6 & -1.64975 & 0.625008 & -0.582336\end{array}$

$\begin{array}{lllll}6 & -0.807592 & 2.909519 & -0.571813\end{array}$

$\begin{array}{llll}6 & -0.522488 & 2.815221 & 0.83072\end{array}$

$\begin{array}{llll}6 & -1.2775 & 0.445695 & 0.848579\end{array}$

$\begin{array}{lllll}6 & -2.097501 & -0.535892 & -1.243337\end{array}$

$\begin{array}{lllll}1 & -0.618424 & 3.844333 & -1.092309\end{array}$

$\begin{array}{llll}1 & -0.121679 & 3.684434 & 1.346563\end{array}$

$\begin{array}{llll}1 & -0.551664 & 1.606385 & 2.58066\end{array}$

$\begin{array}{llll}6 & -0.42576 & -1.650933 & -1.428574\end{array}$

$\begin{array}{lllll}1 & -2.340072 & -0.411112 & -2.301194\end{array}$

$\begin{array}{lllll}6 & -3.073438 & -1.454659 & -0.50812\end{array}$

$\begin{array}{lllll}6 & -2.741212 & -1.639666 & 0.981342\end{array}$

$\begin{array}{lllll}1 & -3.131918 & -2.431707 & -1.005145\end{array}$

$\begin{array}{llll}1 & -4.074865 & -1.0079 & -0.605091\end{array}$

$\begin{array}{llll}1 & -1.913022 & -2.349801 & 1.083506\end{array}$

$\begin{array}{llll}1 & -3.598349 & -2.090435 & 1.497354\end{array}$

$\begin{array}{llll}6 & -2.355529 & -0.31858 & 1.661242\end{array}$

$\begin{array}{llll}1 & -3.236746 & 0.328092 & 1.762392\end{array}$

$\begin{array}{lllll}1 & -0.047051 & -1.165064 & -2.325185\end{array}$

$\begin{array}{lllll}1 & -0.993974 & -2.553262 & -1.644737\end{array}$

$\begin{array}{lllll}6 & 0.439625 & -1.719823 & -0.320085\end{array}$

$6 \quad 1.710846-1.018675-0.184259$

$\begin{array}{llll}1 & 0.271211 & -2.515589 & 0.403103\end{array}$

$\begin{array}{llll}1 & -0.412354 & -0.32509 & 0.755481\end{array}$

$\begin{array}{llll}6 & 4.212415 & 0.273799 & 0.144021\end{array}$

$\begin{array}{lllll}6 & 2.092344 & 0.059553 & -1.012148\end{array}$

$\begin{array}{llll}6 & 2.622438 & -1.419988 & 0.818222\end{array}$

$\begin{array}{llll}6 & 3.851849 & -0.789326 & 0.976855\end{array}$

$\begin{array}{llll}6 & 3.321029 & 0.692836 & -0.847105\end{array}$

$\begin{array}{lllll}1 & 1.412885 & 0.415662 & -1.780562\end{array}$

$\begin{array}{llll}1 & 2.354 & -2.247658 & 1.471755\end{array}$

$\begin{array}{llll}1 & 4.533143 & -1.128271 & 1.753601\end{array}$

$\begin{array}{lllll}1 & 3.584727 & 1.523086 & -1.49792\end{array}$

$\begin{array}{llll}1 & 5.171592 & 0.769187 & 0.267131\end{array}$

$\begin{array}{llll}1 & -1.985806 & -0.510838 & 2.676909\end{array}$

\section{9a)AH model + M: TS Ene 2 RB3LYP/6-31G*}

$\begin{array}{lllll}1 & 0.337614 & 1.395426 & -1.918415\end{array}$

$\begin{array}{lllll}6 & -0.492995 & 1.462543 & -1.22042\end{array}$

$\begin{array}{llll}6 & -2.691789 & 1.655752 & 0.535134\end{array}$

$\begin{array}{lllll}6 & -1.167247 & 0.245681 & -0.84613\end{array}$

$\begin{array}{lllll}6 & -0.915559 & 2.678346 & -0.761653\end{array}$

$\begin{array}{llll}6 & -2.048696 & 2.781042 & 0.11203\end{array}$

$\begin{array}{llll}6 & -2.203011 & 0.329572 & 0.194723\end{array}$

$\begin{array}{llll}6 & -0.732145 & -0.99433 & -1.25509\end{array}$

$\begin{array}{llll}1 & -0.410633 & 3.585381 & -1.084454\end{array}$

$\begin{array}{llll}1 & -2.398538 & 3.763765 & 0.417671\end{array}$

$\begin{array}{llll}1 & -3.563418 & 1.729342 & 1.183177\end{array}$

$\begin{array}{llll}6 & 0.600309 & -1.194772 & 1.06984\end{array}$
$1 \quad 0.123286-1.045242 \quad-1.92642$

$\begin{array}{lllll}6 & -1.57283 & -2.241316 & -1.13072\end{array}$

$\begin{array}{llll}6 & -2.561486 & -2.202443 & 0.048348\end{array}$

$\begin{array}{lllll}1 & -0.933225 & -3.130562 & -1.052705\end{array}$

$\begin{array}{lllll}1 & -2.13118 & -2.371135 & -2.073833\end{array}$

$\begin{array}{lllll}1 & -2.033548 & -2.472867 & 0.96888\end{array}$

$\begin{array}{lllll}1 & -3.338671 & -2.963765 & -0.092047\end{array}$

$\begin{array}{llll}6 & -3.215816 & -0.821957 & 0.221572\end{array}$

$\begin{array}{llll}1 & 0.497657 & -2.256249 & 0.859101\end{array}$

$\begin{array}{lllll}6 & -0.305615 & -0.610403 & 1.96381\end{array}$

$\begin{array}{llll}1 & -0.917289 & -1.28093 & 2.566658\end{array}$

$\begin{array}{llll}1 & -1.352692 & -0.015897 & 1.204867\end{array}$

$\begin{array}{llll}1 & -3.789213 & -0.793667 & 1.157536\end{array}$

$\begin{array}{llll}1 & -0.006794 & 0.296195 & 2.491021\end{array}$

$\begin{array}{llll}6 & 1.833135 & -0.582119 & 0.587616\end{array}$

$\begin{array}{lllll}6 & 2.796382 & -1.380917 & -0.066648\end{array}$

$\begin{array}{lllll}6 & 3.998931 & -0.845381 & -0.514383\end{array}$

$\begin{array}{lllll}6 & 4.277149 & 0.512687 & -0.328836\end{array}$

$\begin{array}{llll}6 & 3.334703 & 1.321253 & 0.309281\end{array}$

$\begin{array}{llll}6 & 2.129794 & 0.786091 & 0.761587\end{array}$

$\begin{array}{lllll}1 & 2.58999 & -2.439267 & -0.213035\end{array}$

$14.724058 \quad-1.487687 \quad-1.007943$

$\begin{array}{lllll}1 & 5.216492 & 0.933419 & -0.677158\end{array}$

$\begin{array}{llll}1 & 3.537856 & 2.379096 & 0.457011\end{array}$

$\begin{array}{llll}1 & 1.410374 & 1.436442 & 1.247978\end{array}$

$\begin{array}{llll}1 & -3.941407 & -0.657769 & -0.588251\end{array}$

\section{0a) $\underline{A H}$ model + M: TS Ene3}

UB3LYP/6-31G*

$\begin{array}{llll}6 & -2.587401 & -1.503828 & 0.839888\end{array}$

$\begin{array}{llll}6 & -1.783615 & -0.307467 & 0.815675\end{array}$

$\begin{array}{llll}6 & -1.631171 & 2.143831 & 0.177451\end{array}$

$\begin{array}{llll}6 & 0.152579 & 1.088579 & 1.686066\end{array}$

$\begin{array}{llll}6 & -0.157438 & 2.145017 & 0.608341\end{array}$

$\begin{array}{llll}6 & -0.590993 & -0.20669 & 1.494363\end{array}$

$\begin{array}{llll}6 & -2.14792 & 0.74088 & -0.146739\end{array}$

$\begin{array}{llll}1 & -1.771736 & 2.805551 & -0.687368\end{array}$

$\begin{array}{llll}1 & 1.233745 & 0.901164 & 1.715016\end{array}$

$\begin{array}{llll}1 & 0.12127 & 3.140873 & 0.974045\end{array}$

$\begin{array}{llll}6 & -3.729894 & -1.60944 & 0.10168\end{array}$

$\begin{array}{lllll}1 & -1.245055 & 0.300297 & -1.109494\end{array}$

$\begin{array}{llll}1 & -5.121317 & -0.606218 & -1.253158\end{array}$

$\begin{array}{llll}1 & -0.102507 & 1.48427 & 2.684292\end{array}$

$\begin{array}{llll}1 & 0.47284 & 1.953696 & -0.265346\end{array}$

$\begin{array}{llll}1 & -0.283599 & -1.041905 & 2.123222\end{array}$

$\begin{array}{lllll}6 & -3.444133 & 0.626824 & -0.793891\end{array}$

$\begin{array}{lllll}1 & -4.329581 & -2.514741 & 0.153711\end{array}$

$\begin{array}{llll}1 & -2.277267 & -2.317541 & 1.492857\end{array}$

$\begin{array}{llll}6 & -4.180052 & -0.517454 & -0.717066\end{array}$

$\begin{array}{lllll}1 & -3.791235 & 1.466654 & -1.393274\end{array}$

$\begin{array}{lllll}6 & 3.942926 & 0.783808 & -0.786264\end{array}$

$\begin{array}{lllll}1 & 4.42352 & 1.646884 & -1.240237\end{array}$

$\begin{array}{llll}1 & 5.682288 & 0.217904 & 0.363562\end{array}$

$\begin{array}{lllll}6 & 4.652093 & -0.017654 & 0.111413\end{array}$

$\begin{array}{llll}1 & 2.219426 & -2.293408 & 0.800794\end{array}$

$14.560533 \quad-1.766075 \quad 1.374238$

$\begin{array}{lllll}6 & 2.623808 & 0.482446 & -1.116674\end{array}$

$\begin{array}{llll}6 & 2.702053 & -1.427579 & 0.352651\end{array}$

$\begin{array}{lllll}1 & 2.105607 & 1.108674 & -1.836931\end{array}$

$\begin{array}{llll}6 & 4.021098 & -1.129523 & 0.677346\end{array}$

$\begin{array}{llll}1 & -0.978732 & -1.02436 & -2.317351\end{array}$
$1 \quad 0.149244 \quad 0.400286 \quad-2.443249$

$\begin{array}{lllll}1 & 0.284342 & -1.976301 & -0.491525\end{array}$

$\begin{array}{llll}6 & 1.964356 & -0.6313 & -0.55221\end{array}$

$\begin{array}{llll}6 & -0.253525 & -0.38809 & -1.807141\end{array}$

$\begin{array}{lllll}6 & 0.597313 & -1.012908 & -0.883138\end{array}$

$\begin{array}{llll}1 & -2.252288 & 2.557586 & 0.985685\end{array}$

49a) $\underline{A H}$ model $+H M \bullet:$ TS tr1a UB3LYP/6-31G*

$\begin{array}{llll}6 & -3.130343 & -1.309217 & 0.285323\end{array}$

$\begin{array}{llll}6 & -2.532725 & -0.001021 & 0.080589\end{array}$

$\begin{array}{llll}6 & -0.867558 & 1.405295 & -1.217608\end{array}$

$\begin{array}{llll}6 & -2.682944 & 2.529112 & 0.168553\end{array}$

$\begin{array}{llll}6 & -1.207799 & 2.561227 & -0.26725\end{array}$

$\begin{array}{llll}6 & -3.177211 & 1.138828 & 0.458804\end{array}$

$\begin{array}{lllll}6 & -1.18858 & 0.046621 & -0.57928\end{array}$

$\begin{array}{llll}1 & -2.829553 & 3.175027 & 1.046304\end{array}$

$1 \quad-0.976812 \quad 3.522944 \quad-0.741209$

$\begin{array}{llll}6 & -2.638643 & -2.417618 & -0.329251\end{array}$

$\begin{array}{llll}1 & -0.349009 & -0.00551 & 0.362038\end{array}$

$\begin{array}{lllll}1 & -1.185916 & -3.208338 & -1.766051\end{array}$

$\begin{array}{lllll}1 & -3.315488 & 2.972185 & -0.619593\end{array}$

$\begin{array}{llll}1 & -0.569279 & 2.490551 & 0.622312\end{array}$

$\begin{array}{llll}1 & -4.145366 & 1.049228 & 0.951164\end{array}$

$\begin{array}{lllll}6 & -0.843261 & -1.146229 & -1.372644\end{array}$

$1-3.126946 \quad-3.379469-0.194726$

$\begin{array}{lllll}1 & -4.033012 & -1.36866 & 0.890293\end{array}$

$\begin{array}{lllll}6 & -1.499257 & -2.325412 & -1.214494\end{array}$

$\begin{array}{lllll}1 & 0.01463 & -1.075797 & -2.03821\end{array}$

$\begin{array}{lllll}1 & 0.481247 & 0.820617 & 2.010876\end{array}$

$\begin{array}{llll}6 & 0.740953 & -0.097115 & 1.480245\end{array}$

$\begin{array}{lllll}6 & 0.281204 & -1.366074 & 2.155859\end{array}$

$\begin{array}{lllll}1 & 0.236282 & -2.210295 & 1.459467\end{array}$

$\begin{array}{llll}1 & 0.957533 & -1.651597 & 2.97615\end{array}$

$\begin{array}{llll}1 & -0.720248 & -1.23795 & 2.577676\end{array}$

$\begin{array}{llll}6 & 1.992618 & -0.031183 & 0.745914\end{array}$

$\begin{array}{llll}6 & 2.613547 & 1.219894 & 0.50712\end{array}$

$\begin{array}{llll}6 & 3.787827 & 1.31783 & -0.229179\end{array}$

$\begin{array}{lllll}6 & 4.387769 & 0.169171 & -0.758442\end{array}$

$\begin{array}{lllll}6 & 3.793488 & -1.076014 & -0.536022\end{array}$

$6 \quad 2.617166 \quad-1.178906 \quad 0.201962$

$\begin{array}{llll}1 & 2.159326 & 2.117996 & 0.920371\end{array}$

$\begin{array}{lllll}1 & 4.243768 & 2.291902 & -0.38847\end{array}$

$\begin{array}{lllll}1 & 5.307099 & 0.244682 & -1.332529\end{array}$

$\begin{array}{lllll}1 & 4.252906 & -1.975471 & -0.938727\end{array}$

$\begin{array}{llll}1 & 2.175998 & -2.157117 & 0.365555\end{array}$

$\begin{array}{llll}1 & 0.192363 & 1.44139 & -1.497339\end{array}$

$\begin{array}{llll}1 & -1.447024 & 1.508545 & -2.146535\end{array}$

51a)AH model + HM•: TS tr $1 b$ UB3LYP/6-31G*

$\begin{array}{llll}6 & 3.24038 & 0.973242 & 0.680363\end{array}$

$\begin{array}{llll}6 & 2.511873 & -0.129032 & 0.076705\end{array}$

$\begin{array}{lllll}6 & 0.748279 & -0.876717 & -1.593387\end{array}$

$\begin{array}{lllll}6 & 2.301348 & -2.582603 & -0.522857\end{array}$

$\begin{array}{lllll}6 & 0.862586 & -2.274762 & -0.97272\end{array}$

$\begin{array}{llll}6 & 2.964557 & -1.41264 & 0.151163\end{array}$

$\begin{array}{lllll}6 & 1.232076 & 0.206987 & -0.623162\end{array}$

$\begin{array}{llll}1 & 2.307587 & -3.455638 & 0.145486\end{array}$

$10.520888 \quad-3.035235 \quad-1.685246$ 


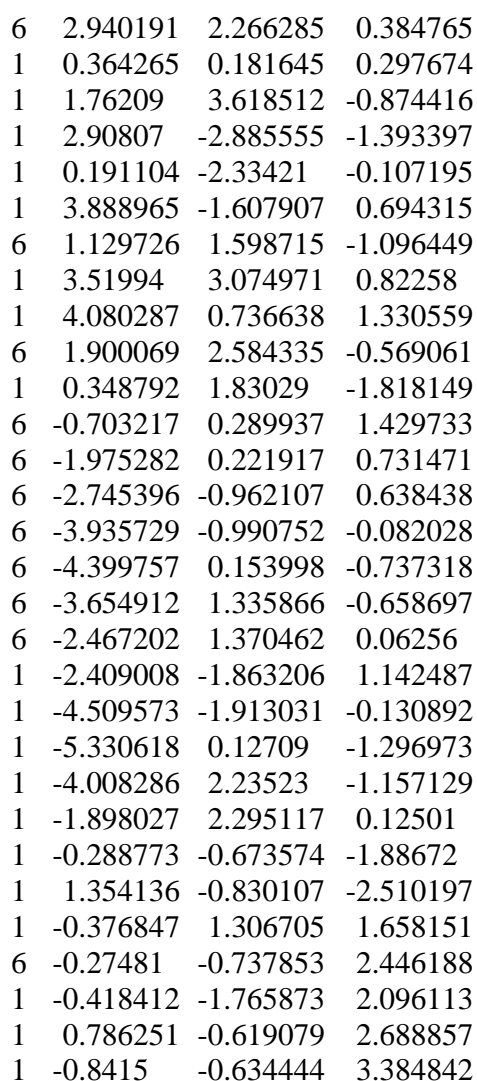

52a) AH model + HM $\bullet$ : TS tr2a UB3LYP/6-31G*

$\begin{array}{lllc}6 & -2.897459 & -1.59916 & 0.238744 \\ 6 & -2.502998 & -0.214665 & 0.043474 \\ 6 & -1.044622 & 1.432982 & -1.21952 \\ 6 & -3.039222 & 2.262844 & 0.125069 \\ 6 & -1.576536 & 2.520024 & -0.275591 \\ 6 & -3.322154 & 0.81281 & 0.406291 \\ 6 & -1.16924 & 0.038414 & -0.590129 \\ 1 & -3.303505 & 2.877079 & 0.998032 \\ 1 & -1.483113 & 3.507967 & -0.742709 \\ 6 & -2.229928 & -2.618184 & -0.364721 \\ 1 & -0.3488 & 0.105036 & 0.364268 \\ 1 & -0.649093 & -3.174742 & -1.775814 \\ 1 & -3.712721 & 2.60569 & -0.678981 \\ 1 & -0.955936 & 2.542242 & 0.629007 \\ 1 & -4.275448 & 0.575807 & 0.878328 \\ 6 & -0.633452 & -1.085264 & -1.379704 \\ 1 & -2.567217 & -3.643762 & -0.237407 \\ 1 & -3.790705 & -1.796805 & 0.828077 \\ 6 & -1.103573 & -2.351061 & -1.230988 \\ 1 & 0.214033 & -0.883648 & -2.031186 \\ 1 & 0.469268 & 1.174523 & 1.88402 \\ 6 & 0.731737 & 0.188631 & 1.496448 \\ 6 & 0.273642 & -0.964072 & 2.356581 \\ 1 & 0.257957 & -1.910786 & 1.806834 \\ 1 & 0.93061 & -1.099411 & 3.229554 \\ 1 & -0.740435 & -0.786948 & 2.728418 \\ 6 & 1.980895 & 0.153418 & 0.757006 \\ 6 & 2.58393 & 1.361164 & 0.325882\end{array}$

$6 \quad 3.755359 \quad 1.360861 \quad-0.421302$

$\begin{array}{lllll}6 & 4.369811 & 0.152495 & -0.770436\end{array}$

$\begin{array}{llll}6 & 3.791799 & -1.051507 & -0.358984\end{array}$

$\begin{array}{llll}6 & 2.617314 & -1.055803 & 0.38929\end{array}$

$\begin{array}{llll}1 & 2.118073 & 2.305866 & 0.598712\end{array}$

$\begin{array}{llll}1 & 4.19754 & 2.304929 & -0.729966\end{array}$

$1 \quad 5.287117 \quad 0.151033 \quad-1.352689$

$14.261693 \quad-1.996011-0.622325$

$\begin{array}{llll}1 & 2.188084 & -2.003315 & 0.699168\end{array}$

$\begin{array}{llll}1 & 0.003535 & 1.62959 & -1.476897\end{array}$

$\begin{array}{lllll}1 & -1.612746 & 1.452099 & -2.160914\end{array}$

53a)AH model $+H M \bullet:$ TS tr $2 b$ UB3LYP/6-31G*

$\begin{array}{lllll}6 & 1.324997 & 0.419754 & -1.037815\end{array}$

$\begin{array}{llll}6 & 2.040137 & 1.527274 & 1.128245\end{array}$

$\begin{array}{lllll}6 & 0.771413 & 2.860157 & -0.628353\end{array}$

$\begin{array}{llll}6 & 0.949193 & 2.575463 & 0.87298\end{array}$

$\begin{array}{lllll}6 & 0.843026 & 1.617998 & -1.471935\end{array}$

$\begin{array}{lllll}6 & 1.739276 & 0.216737 & 0.387202\end{array}$

$\begin{array}{llll}1 & -0.184288 & 3.375476 & -0.79986\end{array}$

$\begin{array}{llll}1 & 1.182246 & 3.504548 & 1.407406\end{array}$

$\begin{array}{llll}6 & 2.19554 & -1.804283 & -1.595871\end{array}$

$\begin{array}{llll}1 & 0.685357 & -0.190991 & 0.951032\end{array}$

$\begin{array}{llll}1 & 3.541237 & -2.69977 & -0.116704\end{array}$

$\begin{array}{lllll}1 & 1.545326 & 3.568195 & -0.970956\end{array}$

$\begin{array}{llll}1 & -0.000505 & 2.207388 & 1.278183\end{array}$

$\begin{array}{llll}1 & 0.528124 & 1.705489 & -2.511319\end{array}$

$\begin{array}{llll}6 & 2.697992 & -0.882559 & 0.599212\end{array}$

$\begin{array}{llll}1 & 2.319012 & -2.62271 & -2.300603\end{array}$

$\begin{array}{llll}1 & 1.017157 & -0.632321 & -2.927318\end{array}$

$\begin{array}{llll}6 & 2.875074 & -1.86534 & -0.321386\end{array}$

$\begin{array}{llll}1 & 3.21506 & -0.92446 & 1.55656\end{array}$

$\begin{array}{llll}6 & -0.593234 & -0.71321 & 1.709792\end{array}$

$\begin{array}{llll}6 & -1.698416 & -0.502628 & 0.789979\end{array}$

$\begin{array}{lllll}6 & -2.015469 & -1.414991 & -0.244323\end{array}$

$\begin{array}{lllll}6 & -3.067932 & -1.170731 & -1.120948\end{array}$

$\begin{array}{llll}6 & -3.840303 & -0.011215 & -1.001995\end{array}$

$\begin{array}{llll}6 & -3.544495 & 0.905755 & 0.012351\end{array}$

$\begin{array}{llll}6 & -2.49425 & 0.664732 & 0.890322\end{array}$

$\begin{array}{lllll}1 & -1.4234 & -2.317085 & -0.363363\end{array}$

$\begin{array}{llll}1 & -3.288747 & -1.890454 & -1.905504\end{array}$

$\begin{array}{lllll}1 & -4.662222 & 0.174407 & -1.687972\end{array}$

$\begin{array}{llll}1 & -4.141872 & 1.807564 & 0.122085\end{array}$

$\begin{array}{llll}1 & -2.283796 & 1.376112 & 1.686281\end{array}$

$\begin{array}{llll}1 & 2.138815 & 1.331877 & 2.204266\end{array}$

$\begin{array}{llll}1 & 3.011658 & 1.914577 & 0.78843\end{array}$

$\begin{array}{llll}1 & -0.56721 & -0.004272 & 2.540248\end{array}$

$\begin{array}{llll}6 & -0.10001 & -2.095023 & 2.062985\end{array}$

$\begin{array}{lllll}1 & 0.131476 & -2.696068 & 1.178038\end{array}$

$\begin{array}{llll}1 & 0.815044 & -2.040329 & 2.662495\end{array}$

$\begin{array}{llll}1 & -0.847981 & -2.646964 & 2.652489\end{array}$

54a) AH model + HM•: TS tr3a UB3LYP/6-31G*

$\begin{array}{llll}6 & -2.275102 & 0.487138 & 1.785934\end{array}$

$\begin{array}{llll}6 & -1.648222 & 0.944542 & 0.558654\end{array}$

$\begin{array}{lllll}6 & -1.396733 & 0.677693 & -1.953226\end{array}$

$\begin{array}{lllll}6 & -0.455327 & 2.730011 & -0.787576\end{array}$ $\begin{array}{llll}6 & -0.218051 & 1.656662 & -1.863234\end{array}$

$\begin{array}{llll}6 & -1.054404 & 2.167977 & 0.47166\end{array}$

$\begin{array}{lllll}6 & -1.666031 & 0.001028 & -0.603663\end{array}$

$\begin{array}{lllll}1 & 0.489187 & 3.242109 & -0.556403\end{array}$

$\begin{array}{llll}1 & -0.046501 & 2.13272 & -2.836485\end{array}$

$\begin{array}{llll}6 & -3.084792 & -0.605676 & 1.80344\end{array}$

$1-0.638571 \quad-0.712764-0.406631$

$\begin{array}{llll}1 & -4.122171 & -2.118605 & 0.604701\end{array}$

$\begin{array}{llll}1 & -1.121497 & 3.51718 & -1.180766\end{array}$

$\begin{array}{lllll}1 & 0.693728 & 1.100138 & -1.617124\end{array}$

$\begin{array}{llll}1 & -1.041266 & 2.803659 & 1.356775\end{array}$

$\begin{array}{llll}6 & -2.751217 & -0.995625 & -0.573976\end{array}$

$\begin{array}{llll}1 & -3.568766 & -0.912247 & 2.727312\end{array}$

$\begin{array}{llll}1 & -2.130892 & 1.081015 & 2.68635\end{array}$

$\begin{array}{llll}6 & -3.376981 & -1.327424 & 0.584468\end{array}$

$\begin{array}{lllll}1 & -2.978487 & -1.526283 & -1.497043\end{array}$

$\begin{array}{llll}1 & -0.038605 & -2.077985 & 0.793842\end{array}$

$\begin{array}{lllll}6 & 0.496375 & -1.735252 & -0.094017\end{array}$

$\begin{array}{lllll}6 & 0.389232 & -2.665283 & -1.276497\end{array}$

$1 \quad 0.70589-2.192789-2.213472$

$1 \quad 1.007133-3.566509-1.142679$

$\begin{array}{llll}1 & -0.646429 & -2.9996 & -1.405744\end{array}$

$\begin{array}{llll}6 & 1.700431 & -0.977946 & 0.193575\end{array}$

$\begin{array}{llll}6 & 1.860414 & -0.369323 & 1.463489\end{array}$

$\begin{array}{llll}6 & 2.986321 & 0.385467 & 1.767906\end{array}$

$\begin{array}{lllll}6 & 3.997313 & 0.563769 & 0.816486\end{array}$

$6 \quad 3.861706 \quad-0.027388-0.443178$

$\begin{array}{lllll}6 & 2.735434 & -0.784411 & -0.753217\end{array}$

$\begin{array}{llll}1 & 1.078337 & -0.501157 & 2.207381\end{array}$

$\begin{array}{llll}1 & 3.081615 & 0.836217 & 2.752714\end{array}$

$\begin{array}{llll}1 & 4.87939 & 1.151545 & 1.055143\end{array}$

$\begin{array}{lllll}1 & 4.643926 & 0.097938 & -1.18802\end{array}$

$\begin{array}{llll}1 & 2.659124 & -1.24374 & -1.734327\end{array}$

$1 \quad-1.204869-0.083864-2.720306$

$\begin{array}{llll}1 & -2.302459 & 1.217272 & -2.26739\end{array}$

55a)AH model + HM $\bullet:$ TS tr $3 b$ UB3LYP/6-31G*

$\begin{array}{lllll}6 & 1.463202 & -0.710844 & -1.937474\end{array}$

$\begin{array}{lllll}6 & 1.324787 & 0.420115 & -1.037621\end{array}$

$\begin{array}{llll}6 & 2.039131 & 1.528497 & 1.128212\end{array}$

$\begin{array}{lllll}6 & 0.769051 & 2.860096 & -0.628433\end{array}$

$\begin{array}{llll}6 & 0.947388 & 2.575847 & 0.872927\end{array}$

$\begin{array}{llll}6 & 0.841846 & 1.617917 & -1.471877\end{array}$

$\begin{array}{llll}6 & 1.739153 & 0.217598 & 0.387441\end{array}$

$\begin{array}{lllll}1 & -0.187259 & 3.374324 & -0.799836\end{array}$

$\begin{array}{llll}1 & 1.179917 & 3.505217 & 1.407082\end{array}$

$6 \quad 2.197195 \quad-1.803258-1.595423$

$\begin{array}{lllll}1 & 0.685485 & -0.190768 & 0.951335\end{array}$

$\begin{array}{lllll}1 & 3.54332 & -2.697634 & -0.115973\end{array}$

$\begin{array}{lllll}1 & 1.542098 & 3.568947 & -0.971306\end{array}$

$\begin{array}{llll}1 & -0.001965 & 2.207198 & 1.278425\end{array}$

$\begin{array}{llll}1 & 0.527006 & 1.705069 & -2.51131\end{array}$

$\begin{array}{llll}6 & 2.698622 & -0.880997 & 0.599671\end{array}$

$\begin{array}{llll}1 & 2.321432 & -2.621623 & -2.300094\end{array}$

$\begin{array}{lllll}1 & 1.018054 & -0.632322 & -2.927099\end{array}$

$\begin{array}{lllll}6 & 2.876578 & -1.863709 & -0.320827\end{array}$

$\begin{array}{llll}1 & 3.215553 & -0.922464 & 1.557113\end{array}$

$\begin{array}{llll}6 & -0.592899 & -0.714243 & 1.709596\end{array}$

$\begin{array}{llll}6 & -1.698112 & -0.5035 & 0.789862\end{array}$

$\begin{array}{lllll}6 & -2.014438 & -1.415203 & -0.245242\end{array}$ 
$6 \begin{array}{llll}6 & -3.066978 & -1.17091 & -1.121765\end{array}$

$\begin{array}{llll}6 & -3.840128 & -0.012007 & -1.001902\end{array}$

$\begin{array}{llll}6 & -3.545025 & 0.904314 & 0.013238\end{array}$

$\begin{array}{llll}6 & -2.494711 & 0.663248 & 0.891122\end{array}$

$\begin{array}{lllll}1 & -1.421699 & -2.316765 & -0.364984\end{array}$

$\begin{array}{lllll}1 & -3.287233 & -1.890109 & -1.90696\end{array}$

$\begin{array}{lllll}1 & -4.662104 & 0.173649 & -1.687801\end{array}$

$\begin{array}{llll}1 & -4.143009 & 1.805636 & 0.123659\end{array}$

$\begin{array}{llll}1 & -2.284798 & 1.374097 & 1.687698\end{array}$

$\begin{array}{llll}1 & 2.138104 & 1.333382 & 2.204252\end{array}$

$\begin{array}{llll}1 & 3.010326 & 1.916438 & 0.788191\end{array}$

$\begin{array}{llll}1 & -0.567286 & -0.005991 & 2.540651\end{array}$

$\begin{array}{llll}6 & -0.0992 & -2.096179 & 2.061646\end{array}$

$\begin{array}{lllll}1 & 0.132982 & -2.696214 & 1.17619\end{array}$

$\begin{array}{llll}1 & 0.815528 & -2.041698 & 2.661661\end{array}$

$\begin{array}{llll}1 & -0.847225 & -2.649057 & 2.650202\end{array}$ 
1b)Styrene monomer RBPW91/6-31G*

$\begin{array}{rrrr}6 & -1.368393 & 1.336131 & -0.000003 \\ 6 & 0.009144 & 1.098408 & -0.000009 \\ 6 & 0.519392 & -0.221041 & -0.000007 \\ 6 & -0.408100 & -1.287562 & 0.000000 \\ 6 & -1.788675 & -1.051355 & 0.000007 \\ 6 & -2.275828 & 0.263108 & 0.000005 \\ 1 & -1.739030 & 2.365460 & -0.000005 \\ 1 & 0.698408 & 1.947105 & -0.000019 \\ 1 & -0.033974 & -2.316729 & 0.000001 \\ 1 & -2.484157 & -1.895719 & 0.000011 \\ 1 & -3.352907 & 0.452782 & 0.000012 \\ 6 & 1.959654 & -0.531428 & -0.000009 \\ 1 & 2.191036 & -1.604364 & -0.000036 \\ 6 & 2.992313 & 0.335784 & 0.000015 \\ 1 & 4.024607 & -0.022132 & 0.000005 \\ 1 & 2.858984 & 1.421329 & 0.000041\end{array}$

\section{4b) $B H x-T S D A$}

RBPW91/6-31G*

$\begin{array}{llll}1 & 3.993627 & 1.998231 & 0.041432\end{array}$ $\begin{array}{lllll}6 & 3.609507 & 0.974666 & -0.025337\end{array}$ $\begin{array}{lllll}6 & 2.636423 & -1.664356 & -0.221388\end{array}$

$\begin{array}{lllll}6 & 2.264560 & 0.782067 & -0.460903\end{array}$

$\begin{array}{lllll}6 & 4.422516 & -0.096140 & 0.303615\end{array}$

$\begin{array}{lllll}6 & 3.930853 & -1.432241 & 0.189704\end{array}$

$\begin{array}{lllll}6 & 1.714387 & -0.569930 & -0.448899\end{array}$

$\begin{array}{lllll}6 & 1.388341 & 1.874968 & -0.675868\end{array}$

$\begin{array}{llll}1 & 5.451649 & 0.076169 & 0.631173\end{array}$

$1 \quad 4.599846-2.271601 \quad 0.402428$

$\begin{array}{lllll}1 & 0.916243 & -0.779150 & -1.171446\end{array}$

$\begin{array}{lllll}1 & 2.272416 & -2.689967 & -0.339799\end{array}$

$\begin{array}{lllll}6 & 0.036877 & 1.686384 & -0.922191\end{array}$

$\begin{array}{lllll}1 & 1.757083 & 2.879524 & -0.434949\end{array}$

$\begin{array}{llll}1 & -0.646997 & 2.539643 & -0.942564\end{array}$

$\begin{array}{lllll}1 & -0.320619 & 0.810359 & -1.466914\end{array}$

$\begin{array}{llll}6 & -0.616500 & 0.424227 & 0.904894\end{array}$

$\begin{array}{llll}6 & 0.433922 & -0.526789 & 1.071868\end{array}$

$\begin{array}{llll}1 & 0.150700 & -1.583648 & 1.037884\end{array}$

$\begin{array}{lllll}1 & 1.166776 & -0.312306 & 1.853707\end{array}$

$\begin{array}{llll}1 & -0.555031 & 1.344029 & 1.491481\end{array}$

$\begin{array}{llll}6 & -1.970257 & 0.064237 & 0.455324\end{array}$

$\begin{array}{lllll}6 & -2.248934 & -1.111436 & -0.284858\end{array}$

$\begin{array}{lllll}6 & -3.552093 & -1.416338 & -0.695369\end{array}$

$\begin{array}{lllll}6 & -4.617774 & -0.559171 & -0.379722\end{array}$

$\begin{array}{llll}6 & -4.361243 & 0.612960 & 0.349333\end{array}$

$\begin{array}{llll}6 & -3.058357 & 0.921728 & 0.753147\end{array}$

$\begin{array}{lllll} & -1.436591 & -1.797985 & -0.541481\end{array}$

$\begin{array}{lllll}1 & -3.736928 & -2.332037 & -1.265214\end{array}$

$\begin{array}{lllll}1 & -5.635275 & -0.801265 & -0.698726\end{array}$

$\begin{array}{llll}1 & -5.181406 & 1.291103 & 0.603701\end{array}$

$\begin{array}{llll}1 & -2.868859 & 1.839882 & 1.319185\end{array}$

\section{5b)BHn-TSDA}

RBPW91/6-31G*

$\begin{array}{llll}6 & 3.744872 & 0.988278 & 0.073862\end{array}$

$\begin{array}{lllll}6 & 2.305810 & 0.805211 & -0.076396\end{array}$

$\begin{array}{lllll}6 & 0.361956 & -0.817247 & -0.000530\end{array}$ $\begin{array}{llll}6 & -0.070534 & 1.685297 & 0.116604\end{array}$

$\begin{array}{lllll}6 & -0.555907 & 0.335113 & -0.474315\end{array}$

$\begin{array}{llll}6 & 1.429294 & 1.821474 & 0.143839\end{array}$

$\begin{array}{llll}6 & 1.810662 & -0.586344 & -0.479930\end{array}$

$\begin{array}{lllll}1 & -0.016265 & -1.779988 & -0.386658\end{array}$

$\begin{array}{lllll}1 & -0.523967 & 2.518554 & -0.453528\end{array}$

$\begin{array}{lllll}6 & -2.029213 & 0.075344 & -0.189785\end{array}$

$\begin{array}{llll}6 & 4.588403 & -0.077112 & 0.182603\end{array}$

$\begin{array}{lllll}1 & 1.780705 & -0.605459 & -1.595514\end{array}$

$1 \quad 4.759704 \quad-2.265098 \quad 0.400132$

$\begin{array}{llll}1 & 0.350573 & -0.884859 & 1.102112\end{array}$

$\begin{array}{llll}1 & -0.461619 & 1.805750 & 1.147756\end{array}$

$\begin{array}{lllll}1 & -0.440140 & 0.400586 & -1.572988\end{array}$

$\begin{array}{llll}1 & 1.828131 & 2.811662 & 0.399263\end{array}$

$\begin{array}{lllll}6 & 2.768185 & -1.693017 & -0.086674\end{array}$

$\begin{array}{llll}1 & 5.659807 & 0.081768 & 0.336205\end{array}$

$\begin{array}{lllll}1 & 4.125408 & 2.010766 & 0.172204\end{array}$

$\begin{array}{llll}6 & 4.071935 & -1.443422 & 0.176748\end{array}$

$\begin{array}{lllll}1 & 2.380878 & -2.717874 & -0.095541\end{array}$

$\begin{array}{llll}6 & -4.775380 & -0.416887 & 0.314981\end{array}$

$\begin{array}{llll}6 & -2.503292 & -0.109588 & 1.125743\end{array}$

$\begin{array}{lllll}6 & -2.960571 & 0.008521 & -1.243581\end{array}$

$\begin{array}{lllll}6 & -4.320189 & -0.234415 & -0.998157\end{array}$

$\begin{array}{llll}6 & -3.860098 & -0.353943 & 1.376890\end{array}$

$\begin{array}{llll}1 & -1.803629 & -0.064709 & 1.966477\end{array}$

$\begin{array}{llll}1 & -2.613486 & 0.149149 & -2.272923\end{array}$

$\begin{array}{lllll}1 & -5.023238 & -0.281019 & -1.835186\end{array}$

$\begin{array}{llll}1 & -4.203900 & -0.495991 & 2.405872\end{array}$

$\begin{array}{llll}1 & -5.834568 & -0.606812 & 0.510909\end{array}$

\section{6b)TS1 (At)}

\section{UBPW91/6-31G*}

$\begin{array}{llll}1 & -4.677627 & 2.503514 & 0.137821\end{array}$

$\begin{array}{llll}6 & -4.479656 & 1.428418 & 0.089356\end{array}$

$\begin{array}{llll}6 & -3.968494 & -1.320616 & -0.044272\end{array}$

$\begin{array}{llll}6 & -3.189101 & 0.956341 & 0.330643\end{array}$

$\begin{array}{llll}6 & -5.525072 & 0.537329 & -0.213640\end{array}$

$\begin{array}{lllll}6 & -5.257220 & -0.842269 & -0.276559\end{array}$

$\begin{array}{lllll}6 & -2.890336 & -0.439284 & 0.274000\end{array}$

$\begin{array}{lllll}1 & -2.395258 & 1.669764 & 0.567233\end{array}$

$\begin{array}{lllll}1 & -6.534617 & 0.913577 & -0.399677\end{array}$

$\begin{array}{lllll}1 & -6.061430 & -1.545134 & -0.513899\end{array}$

$\begin{array}{llll}6 & -1.586429 & -0.977973 & 0.526161\end{array}$

$\begin{array}{lllll}1 & -3.767802 & -2.395578 & -0.099194\end{array}$

$\begin{array}{llll}6 & -0.386995 & -0.229717 & 0.769313\end{array}$

$\begin{array}{lllll}1 & -1.476449 & -2.056264 & 0.355815\end{array}$

$\begin{array}{llll}1 & 6.534628 & -0.913553 & 0.399665\end{array}$

$\begin{array}{lllll}6 & 0.386991 & 0.229696 & -0.769306\end{array}$

$\begin{array}{lllll}6 & 1.586422 & 0.977959 & -0.526155\end{array}$

$\begin{array}{lllll}1 & 1.476435 & 2.056250 & -0.355810\end{array}$

$14.677647 \quad-2.503505-0.137822$

$\begin{array}{lllll}6 & 2.890333 & 0.439280 & -0.273997\end{array}$

$\begin{array}{llll}6 & 5.525079 & -0.537313 & 0.213632\end{array}$

$\begin{array}{llll}6 & 3.189109 & -0.956343 & -0.330639\end{array}$

$\begin{array}{llll}6 & 3.968485 & 1.320620 & 0.044270\end{array}$

$\begin{array}{llll}6 & 5.257217 & 0.842283 & 0.276551\end{array}$

$\begin{array}{lllll}6 & 4.479668 & -1.428410 & -0.089357\end{array}$

$\begin{array}{lllll}1 & 2.395269 & -1.669772 & -0.567225\end{array}$

$\begin{array}{llll}1 & 3.767786 & 2.395581 & 0.099191\end{array}$

$\begin{array}{llll}1 & 6.061422 & 1.545155 & 0.513887\end{array}$

$\begin{array}{lllll}1 & 0.528505 & -0.736080 & -1.270427\end{array}$
$1-0.407758 \quad 0.804165 \quad-1.262240$

$\begin{array}{llll}1 & 0.407752 & -0.804190 & 1.262245\end{array}$

$\begin{array}{llll}1 & -0.528504 & 0.736059 & 1.270436\end{array}$

7b) $\underline{T S 1}(\mathrm{Ac})$

UBPW91/6-31G*

$\begin{array}{llll}1 & -2.840669 & 2.883194 & 0.280404\end{array}$

$\begin{array}{llll}6 & -3.189671 & 1.853056 & 0.160710\end{array}$

$\begin{array}{lllll}6 & -4.081435 & -0.783255 & -0.148464\end{array}$

$\begin{array}{lllll}6 & -2.277350 & 0.853521 & -0.180743\end{array}$

$\begin{array}{llll}6 & -4.549891 & 1.548729 & 0.347582\end{array}$

$\begin{array}{llll}6 & -4.987739 & 0.221101 & 0.186446\end{array}$

$\begin{array}{llll}6 & -2.694410 & -0.502793 & -0.345817\end{array}$

$\begin{array}{llll}1 & -1.228455 & 1.117982 & -0.339628\end{array}$

$\begin{array}{llll}1 & -5.259831 & 2.336160 & 0.614519\end{array}$

$\begin{array}{llll}1 & -6.043764 & -0.027133 & 0.328781\end{array}$

$\begin{array}{lllll}6 & -1.799888 & -1.562727 & -0.701697\end{array}$

$\begin{array}{lllll}1 & -4.429296 & -1.814622 & -0.267107\end{array}$

$\begin{array}{llll}6 & -0.369523 & -1.471698 & -0.802810\end{array}$

$\begin{array}{lllll}1 & -2.240649 & -2.566399 & -0.737523\end{array}$

$\begin{array}{llll}1 & 5.259855 & 2.336120 & -0.614595\end{array}$

$\begin{array}{lllll}6 & 0.369506 & -1.471674 & 0.802848\end{array}$

$\begin{array}{llll}6 & 1.799873 & -1.562698 & 0.701740\end{array}$

$\begin{array}{lllll}1 & 2.240635 & -2.566370 & 0.737601\end{array}$

$\begin{array}{llll}1 & 2.840726 & 2.883216 & -0.280340\end{array}$

$\begin{array}{llll}6 & 2.694403 & -0.502776 & 0.345836\end{array}$

$\begin{array}{lllll}6 & 4.549907 & 1.548705 & -0.347631\end{array}$

$\begin{array}{llll}6 & 2.277375 & 0.853552 & 0.180805\end{array}$

$\begin{array}{llll}6 & 4.081411 & -0.783272 & 0.148410\end{array}$

$\begin{array}{lllll}6 & 4.987726 & 0.221062 & -0.186534\end{array}$

$\begin{array}{lllll}6 & 3.189706 & 1.853066 & -0.160682\end{array}$

$\begin{array}{llll}1 & 1.228497 & 1.118047 & 0.339751\end{array}$

$1 \quad 4.429250 \quad-1.814650 \quad 0.267019$

$\begin{array}{llll}1 & 6.043737 & -0.027200 & -0.328925\end{array}$

$\begin{array}{llll}1 & -0.027629 & -0.534443 & 1.219204\end{array}$

$\begin{array}{llll}1 & -0.084468 & -2.330404 & 1.311681\end{array}$

$\begin{array}{lllll}1 & 0.027600 & -0.534475 & -1.219195\end{array}$

$1 \quad 0.084456 \quad-2.330437 \quad-1.311625$

8b)TS1 (Gs)

UBPW91/6-31G*

$\begin{array}{llll}1 & -4.412060 & 0.779123 & -1.688183\end{array}$

$\begin{array}{lllll}6 & -3.809589 & 0.237697 & -0.952394\end{array}$

$\begin{array}{llll}6 & -2.264115 & -1.141828 & 0.930770\end{array}$

$\begin{array}{lllll}6 & -2.700728 & 0.863859 & -0.378506\end{array}$

$\begin{array}{lllll}6 & -4.160766 & -1.073414 & -0.588636\end{array}$

$\begin{array}{llll}6 & -3.378083 & -1.756153 & 0.361598\end{array}$

$\begin{array}{llll}6 & -1.887101 & 0.191863 & 0.583955\end{array}$

$\begin{array}{lllll}1 & -2.461654 & 1.890778 & -0.667881\end{array}$

$\begin{array}{lllll}1 & -5.033364 & -1.556557 & -1.036387\end{array}$

$\begin{array}{llll}1 & -3.641730 & -2.776979 & 0.654454\end{array}$

$\begin{array}{llll}6 & -0.734425 & 0.790496 & 1.194915\end{array}$

$\begin{array}{llll}1 & -1.656358 & -1.683528 & 1.662676\end{array}$

$\begin{array}{llll}6 & -0.265450 & 2.135622 & 0.951071\end{array}$

$\begin{array}{llll}1 & -0.163644 & 0.159712 & 1.882836\end{array}$

$\begin{array}{lllll}1 & 3.427240 & -3.248407 & 0.037244\end{array}$

$\begin{array}{lllll}6 & 0.641759 & 2.358083 & -0.508034\end{array}$

$\begin{array}{lllll}6 & 2.004278 & 1.899795 & -0.397685\end{array}$

$\begin{array}{lllll}1 & 2.772140 & 2.637128 & -0.136244\end{array}$

$1 \quad 1.082720 \quad-2.653651 \quad-0.593685$ 
$\begin{array}{llll}6 & 2.381496 & 0.531830 & -0.344462\end{array}$

$\begin{array}{lllll}6 & 3.134270 & -2.199903 & -0.066463\end{array}$

$\begin{array}{lllll}6 & 1.440385 & -0.524361 & -0.566915\end{array}$

$\begin{array}{llll}6 & 3.721050 & 0.157994 & 0.001577\end{array}$

$\begin{array}{lllll}6 & 4.086831 & -1.175309 & 0.133965\end{array}$

$\begin{array}{lllll}6 & 1.819717 & -1.864756 & -0.416553\end{array}$

$\begin{array}{lllll}1 & 0.431923 & -0.289232 & -0.908328\end{array}$

$\begin{array}{llll}1 & 4.457523 & 0.949556 & 0.174727\end{array}$

$\begin{array}{llll}1 & 5.116048 & -1.433486 & 0.400800\end{array}$

$\begin{array}{lllll}1 & 0.021391 & 1.828151 & -1.247419\end{array}$

$\begin{array}{lllll}1 & 0.581225 & 3.440194 & -0.692407\end{array}$

$\begin{array}{llll}1 & 0.385631 & 2.516900 & 1.747302\end{array}$

$\begin{array}{llll}1 & -1.084201 & 2.851209 & 0.781265\end{array}$

\section{9b)TS1 (Gu)}

UBPW91/6-31G*

$1 \quad 2.285276-1.247316-2.597155$

$\begin{array}{lllll}6 & 2.310653 & -1.057325 & -1.519824\end{array}$

$\begin{array}{llll}6 & 2.372222 & -0.572656 & 1.238319\end{array}$

$\begin{array}{lllll}6 & 1.857095 & 0.168210 & -1.026653\end{array}$

$\begin{array}{lllll}6 & 2.806854 & -2.040684 & -0.648171\end{array}$

$\begin{array}{llll}6 & 2.837154 & -1.785301 & 0.736864\end{array}$

$\begin{array}{llll}6 & 1.865449 & 0.446029 & 0.373967\end{array}$

$\begin{array}{lllll}1 & 1.488008 & 0.922193 & -1.725366\end{array}$

$\begin{array}{lllll}1 & 3.171485 & -2.993672 & -1.041237\end{array}$

$\begin{array}{llll}1 & 3.222322 & -2.544799 & 1.423905\end{array}$

$\begin{array}{llll}6 & 1.399290 & 1.684625 & 0.918784\end{array}$

$\begin{array}{llll}1 & 2.387934 & -0.385196 & 2.317012\end{array}$

$\begin{array}{llll}6 & 0.879651 & 2.780576 & 0.153901\end{array}$

$\begin{array}{llll}1 & 1.380611 & 1.765457 & 2.011766\end{array}$

$\begin{array}{llll}1 & -3.185074 & -2.983559 & 1.042250\end{array}$

$\begin{array}{llll}6 & -0.870010 & 2.781423 & -0.154884\end{array}$

$\begin{array}{lllll}6 & -1.391206 & 1.685919 & -0.919376\end{array}$

$\begin{array}{lllll}1 & -1.370534 & 1.765503 & -2.012414\end{array}$

$\begin{array}{llll}1 & -2.293369 & -1.239688 & 2.597862\end{array}$

$\begin{array}{lllll}6 & -1.862659 & 0.449465 & -0.374056\end{array}$

$\begin{array}{llll}6 & -2.815880 & -2.032461 & 0.648865\end{array}$

$\begin{array}{llll}6 & -1.857257 & 0.172718 & 1.026772\end{array}$

$\begin{array}{lllll}6 & -2.372423 & -0.567852 & -1.238227\end{array}$

$\begin{array}{llll}6 & -2.843161 & -1.778129 & -0.736395\end{array}$

$\begin{array}{lllll}6 & -2.316622 & -1.050460 & 1.520348\end{array}$

$\begin{array}{llll}1 & -1.485542 & 0.925566 & 1.725330\end{array}$

$\begin{array}{lllll}1 & -2.385933 & -0.381241 & -2.317097\end{array}$

$1-3.230577 \quad-2.536561 \quad-1.423351$

$\begin{array}{llll}1 & -1.281766 & 2.872130 & 0.858718\end{array}$

$\begin{array}{lllll}1 & -0.956520 & 3.743572 & -0.678724\end{array}$

$\begin{array}{lllll}1 & 1.291045 & 2.870088 & -0.859967\end{array}$

$\begin{array}{lllll}1 & 0.967741 & 3.742936 & 0.677067\end{array}$

\section{0b)TS1 (Gw)}

UBPW91/6-31G*

$1 \quad 5.012768 \quad-2.038110 \quad 1.018052$

$\begin{array}{llll}6 & 4.653223 & -1.109745 & 0.563834\end{array}$

$\begin{array}{llll}6 & 3.729477 & 1.264677 & -0.594835\end{array}$

$\begin{array}{llll}6 & 3.329942 & -1.025432 & 0.130090\end{array}$

$\begin{array}{llll}6 & 5.527428 & -0.017015 & 0.419380\end{array}$

$\begin{array}{llll}6 & 5.052839 & 1.170660 & -0.166179\end{array}$

$\begin{array}{llll}6 & 2.821371 & 0.169723 & -0.464369\end{array}$

$\begin{array}{llll}1 & 2.674372 & -1.892464 & 0.248255\end{array}$

$\begin{array}{llll}1 & 6.564454 & -0.091042 & 0.757667\end{array}$
$1 \quad 5.722354 \quad 2.028054 \quad-0.283492$

$\begin{array}{lllll}6 & 1.469679 & 0.315404 & -0.919925\end{array}$

$\begin{array}{lllll}1 & 3.367141 & 2.194598 & -1.045163\end{array}$

$\begin{array}{llll}6 & 0.421005 & -0.651872 & -0.793726\end{array}$

$\begin{array}{lllll}1 & 1.196638 & 1.312079 & -1.286894\end{array}$

$\begin{array}{llll}1 & -6.564042 & -0.091230 & -0.758954\end{array}$

$\begin{array}{llll}6 & -0.421197 & -0.651702 & 0.794714\end{array}$

$\begin{array}{llll}6 & -1.469965 & 0.315508 & 0.920672\end{array}$

$\begin{array}{llll}1 & -1.197119 & 1.312170 & 1.287814\end{array}$

$\begin{array}{llll}1 & -5.012081 & -2.038137 & -1.018892\end{array}$

$\begin{array}{llll}6 & -2.821480 & 0.169755 & 0.464620\end{array}$

$\begin{array}{llll}6 & -5.527160 & -0.017145 & -0.420238\end{array}$

$\begin{array}{lllll}6 & -3.329702 & -1.025391 & -0.130157\end{array}$

$\begin{array}{llll}6 & -3.729736 & 1.264615 & 0.594830\end{array}$

$\begin{array}{llll}6 & -5.052914 & 1.170519 & 0.165628\end{array}$

$\begin{array}{lllll}6 & -4.652802 & -1.109783 & -0.564442\end{array}$

$\begin{array}{lllll}1 & -2.674003 & -1.892351 & -0.248138\end{array}$

$\begin{array}{llll}1 & -3.367664 & 2.194527 & 1.045387\end{array}$

$\begin{array}{llll}1 & -5.722556 & 2.027841 & 0.282749\end{array}$

$\begin{array}{llll}1 & -0.768894 & -1.693912 & 0.813567\end{array}$

$\begin{array}{llll}1 & 0.410164 & -0.517118 & 1.497457\end{array}$

$\begin{array}{llll}1 & 0.768809 & -1.694052 & -0.812325\end{array}$

$\begin{array}{llll}1 & -0.410390 & -0.517524 & -1.496472\end{array}$

$11 b) \cdot M 2 \cdot(A t)$

UBPW91/6-31G*

$\begin{array}{llll}1 & -4.697761 & 2.507003 & 0.117707\end{array}$

$\begin{array}{llll}6 & -4.501309 & 1.431301 & 0.076658\end{array}$

$\begin{array}{llll}6 & -3.999082 & -1.322838 & -0.035502\end{array}$

$\begin{array}{llll}6 & -3.206877 & 0.961535 & 0.295374\end{array}$

$\begin{array}{lllll}6 & -5.554318 & 0.537904 & -0.195002\end{array}$

$\begin{array}{llll}6 & -5.289374 & -0.844316 & -0.247725\end{array}$

$\begin{array}{llll}6 & -2.908851 & -0.438040 & 0.248150\end{array}$

$\begin{array}{llll}1 & -2.406927 & 1.676042 & 0.505921\end{array}$

$\begin{array}{llll}1 & -6.567078 & 0.913310 & -0.364160\end{array}$

$\begin{array}{lllll}1 & -6.099984 & -1.547889 & -0.460016\end{array}$

$\begin{array}{lllll}6 & -1.607296 & -0.969724 & 0.474848\end{array}$

$\begin{array}{lllll}1 & -3.801111 & -2.398707 & -0.080726\end{array}$

$\begin{array}{llll}6 & -0.369523 & -0.191882 & 0.695385\end{array}$

$\begin{array}{llll}1 & -1.494637 & -2.052811 & 0.344139\end{array}$

$\begin{array}{llll}1 & 6.567091 & -0.913296 & 0.364112\end{array}$

$\begin{array}{lllll}6 & 0.369516 & 0.191871 & -0.695355\end{array}$

$\begin{array}{lllll}6 & 1.607287 & 0.969716 & -0.474819\end{array}$

$\begin{array}{lllll}1 & 1.494624 & 2.052801 & -0.344097\end{array}$

$14.697779-2.506996-0.117748$

$\begin{array}{lllll}6 & 2.908848 & 0.438037 & -0.248139\end{array}$

$\begin{array}{llll}6 & 5.554327 & -0.537895 & 0.194970\end{array}$

$\begin{array}{lllll}6 & 3.206882 & -0.961536 & -0.295381\end{array}$

$\begin{array}{llll}6 & 3.999077 & 1.322839 & 0.035509\end{array}$

$\begin{array}{llll}6 & 5.289374 & 0.844324 & 0.247711\end{array}$

$\begin{array}{lllll}6 & 4.501320 & -1.431296 & -0.076686\end{array}$

$\begin{array}{lllll}1 & 2.406934 & -1.676046 & -0.505925\end{array}$

$\begin{array}{llll}1 & 3.801099 & 2.398706 & 0.080746\end{array}$

$\begin{array}{llll}1 & 6.099982 & 1.547900 & 0.459999\end{array}$

$\begin{array}{lllll}1 & 0.555868 & -0.750611 & -1.234376\end{array}$

$\begin{array}{lllll}1 & -0.358963 & 0.770957 & -1.285223\end{array}$

$\begin{array}{llll}1 & 0.358954 & -0.770968 & 1.285255\end{array}$

$\begin{array}{llll}1 & -0.555876 & 0.750601 & 1.234406\end{array}$

$12 b) \cdot M 2 \cdot(A c)$

UBPW91/6-31G*
$1 \quad-2.671269 \quad 2.909431 \quad-0.181629$

$\begin{array}{llll}6 & -3.069869 & 1.892618 & -0.113591\end{array}$

$\begin{array}{lllll}6 & -4.092066 & -0.710605 & 0.060198\end{array}$

$\begin{array}{llll}6 & -2.220897 & 0.807212 & -0.326033\end{array}$

$\begin{array}{llll}6 & -4.430688 & 1.693154 & 0.184692\end{array}$

$\begin{array}{llll}6 & -4.932885 & 0.379947 & 0.267516\end{array}$

$\begin{array}{lllll}6 & -2.703611 & -0.538329 & -0.246504\end{array}$

$\begin{array}{lllll}1 & -1.170012 & 0.988551 & -0.565905\end{array}$

$\begin{array}{llll}1 & -5.090847 & 2.548681 & 0.350437\end{array}$

$\begin{array}{llll}1 & -5.989248 & 0.213064 & 0.498622\end{array}$

$\begin{array}{lllll}6 & -1.879520 & -1.678299 & -0.469739\end{array}$

$\begin{array}{llll}1 & -4.490436 & -1.728161 & 0.128172\end{array}$

$\begin{array}{lllll}6 & -0.418291 & -1.667339 & -0.695130\end{array}$

$\begin{array}{lllll}1 & -2.354844 & -2.657450 & -0.336256\end{array}$

$\begin{array}{lllll}1 & 5.090956 & 2.548588 & -0.350473\end{array}$

$\begin{array}{llll}6 & 0.418226 & -1.667255 & 0.695158\end{array}$

$\begin{array}{lllll}6 & 1.879451 & -1.678248 & 0.469757\end{array}$

$\begin{array}{llll}1 & 2.354763 & -2.657415 & 0.336346\end{array}$

$\begin{array}{llll}1 & 2.671401 & 2.909451 & 0.181627\end{array}$

$\begin{array}{llll}6 & 2.703587 & -0.538308 & 0.246516\end{array}$

$\begin{array}{lllll}6 & 4.430759 & 1.693092 & -0.184718\end{array}$

$\begin{array}{llll}6 & 2.220934 & 0.807255 & 0.326042\end{array}$

$\begin{array}{lllll}6 & 4.092025 & -0.710650 & -0.060212\end{array}$

$\begin{array}{lllll}6 & 4.932893 & 0.379863 & -0.267548\end{array}$

$\begin{array}{llll}6 & 3.069954 & 1.892620 & 0.113585\end{array}$

$\begin{array}{llll}1 & 1.170061 & 0.988647 & 0.565926\end{array}$

$14.490348 \quad-1.728224 \quad-0.128197$

$\begin{array}{lllll}1 & 5.989244 & 0.212929 & -0.498673\end{array}$

$\begin{array}{llll}1 & 0.083444 & -0.783936 & 1.262567\end{array}$

$1 \quad 0.103952 \quad-2.560135 \quad 1.258413$

$\begin{array}{lllll}1 & -0.083504 & -0.784093 & -1.262647\end{array}$

$\begin{array}{lllll}1 & -0.104023 & -2.560296 & -1.258267\end{array}$

13b) $\cdot M 2 \cdot(G s)$

UBPW91/6-31G*

$1 \quad-4.510738 \quad 0.627430 \quad-1.726878$

$\begin{array}{lllll}6 & -3.894391 & 0.137164 & -0.967099\end{array}$

$\begin{array}{llll}6 & -2.314447 & -1.116441 & 0.976804\end{array}$

$\begin{array}{lllll}6 & -2.800072 & 0.815550 & -0.429065\end{array}$

$\begin{array}{llll}6 & -4.213877 & -1.164181 & -0.539946\end{array}$

$\begin{array}{llll}6 & -3.413053 & -1.782928 & 0.439520\end{array}$

$\begin{array}{llll}6 & -1.967451 & 0.209711 & 0.564296\end{array}$

$\begin{array}{lllll}1 & -2.581171 & 1.830268 & -0.771934\end{array}$

$\begin{array}{lllll}1 & -5.075232 & -1.688668 & -0.962135\end{array}$

$\begin{array}{lllll}1 & -3.651876 & -2.794887 & 0.780439\end{array}$

$\begin{array}{llll}6 & -0.834593 & 0.860142 & 1.142814\end{array}$

$\begin{array}{llll}1 & -1.693777 & -1.607289 & 1.733297\end{array}$

$\begin{array}{llll}6 & -0.363349 & 2.213314 & 0.804646\end{array}$

$\begin{array}{llll}1 & -0.250652 & 0.279942 & 1.864100\end{array}$

$\begin{array}{llll}1 & 3.687231 & -3.190868 & -0.028120\end{array}$

$\begin{array}{lllll}6 & 0.585569 & 2.318547 & -0.530033\end{array}$

$\begin{array}{lllll}6 & 1.974274 & 1.881986 & -0.324901\end{array}$

$\begin{array}{lllll}1 & 2.704407 & 2.649047 & -0.041824\end{array}$

$\begin{array}{lllll}1 & 1.321204 & -2.713111 & -0.685438\end{array}$

$\begin{array}{lllll}6 & 2.413814 & 0.532735 & -0.282684\end{array}$

$\begin{array}{lllll}6 & 3.333134 & -2.158598 & -0.096967\end{array}$

$\begin{array}{lllll}6 & 1.546612 & -0.571564 & -0.566681\end{array}$

$\begin{array}{llll}6 & 3.765821 & 0.226171 & 0.082744\end{array}$

$\begin{array}{llll}6 & 4.212144 & -1.087560 & 0.170959\end{array}$

$\begin{array}{lllll}6 & 2.004803 & -1.887950 & -0.464289\end{array}$

$10.520139 \quad-0.383903 \quad-0.886202$ 
$\begin{array}{llll}1 & 4.448936 & 1.053320 & 0.302038\end{array}$ $\begin{array}{llll}1 & 5.249409 & -1.291296 & 0.453446\end{array}$ $\begin{array}{lllll}1 & 0.059833 & 1.747378 & -1.313035\end{array}$ $\begin{array}{lllll}1 & 0.563410 & 3.381628 & -0.818706\end{array}$ $\begin{array}{lllll}1 & 0.207660 & 2.656943 & 1.633714\end{array}$ $\begin{array}{llll}1 & -1.208428 & 2.887480 & 0.586537\end{array}$

14b) $M 2 \cdot(G n)$ UBPW91/6-31G*

$\begin{array}{llll}1 & -2.493597 & -1.288462 & 2.585681\end{array}$

$\begin{array}{llll}6 & -2.506886 & -1.047365 & 1.518379\end{array}$

$\begin{array}{lllll}6 & -2.533538 & -0.437086 & -1.215550\end{array}$

$\begin{array}{llll}6 & -1.924742 & 0.140321 & 1.076429\end{array}$

$\begin{array}{llll}6 & -3.111013 & -1.934508 & 0.609035\end{array}$

$\begin{array}{lllll}6 & -3.118720 & -1.615974 & -0.763425\end{array}$

$\begin{array}{lllll}6 & -1.915454 & 0.487491 & -0.312095\end{array}$

$\begin{array}{llll}1 & -1.460248 & 0.811040 & 1.802880\end{array}$

$\begin{array}{llll}1 & -3.570397 & -2.861140 & 0.963570\end{array}$

$\begin{array}{lllll}1 & -3.584520 & -2.299296 & -1.479955\end{array}$

$\begin{array}{lllll}6 & -1.343990 & 1.690762 & -0.812810\end{array}$

$\begin{array}{lllll}1 & -2.540714 & -0.198514 & -2.284192\end{array}$

$\begin{array}{lllll}6 & -0.698328 & 2.759118 & -0.020952\end{array}$

$\begin{array}{lllll}1 & -1.362337 & 1.827921 & -1.900727\end{array}$

$1 \quad 3.239042 \quad-3.113456-0.915700$

$\begin{array}{lllll}6 & 0.917758 & 2.757332 & -0.021094\end{array}$

$\begin{array}{llll}6 & 1.552797 & 1.691245 & 0.782648\end{array}$

$\begin{array}{llll}1 & 1.646099 & 1.871856 & 1.860043\end{array}$

$\begin{array}{llll}1 & 2.219394 & -1.507105 & -2.541190\end{array}$

$\begin{array}{llll}6 & 1.987131 & 0.424001 & 0.305629\end{array}$

$\begin{array}{lllll}6 & 2.891173 & -2.134046 & -0.576393\end{array}$

$\begin{array}{lllll}6 & 1.880401 & 0.026406 & -1.065372\end{array}$

$\begin{array}{llll}6 & 2.572222 & -0.519554 & 1.211687\end{array}$

$\begin{array}{lllll}6 & 3.014609 & -1.765457 & 0.778374\end{array}$

$\begin{array}{lllll}6 & 2.319337 & -1.228413 & -1.487765\end{array}$

$\begin{array}{lllll}1 & 1.442275 & 0.713635 & -1.792488\end{array}$

$\begin{array}{llll}1 & 2.666700 & -0.242325 & 2.266765\end{array}$

$\begin{array}{llll}1 & 3.457944 & -2.462972 & 1.495458\end{array}$

$\begin{array}{llll}1 & 1.243243 & 2.739743 & -1.073804\end{array}$

$\begin{array}{llll}1 & 1.200196 & 3.742599 & 0.388177\end{array}$

$\begin{array}{llll}1 & -1.027418 & 2.759312 & 1.030031\end{array}$

$\begin{array}{lllll}1 & -0.972211 & 3.741523 & -0.444890\end{array}$

15b) $\cdot M 2 \cdot(G w)$

UBPW91/6-31G*

$\begin{array}{llll}1 & -5.267340 & -1.906237 & 0.831068\end{array}$

$\begin{array}{lllll}6 & -4.788910 & -1.000351 & 0.446383\end{array}$

$\begin{array}{llll}6 & -3.565025 & 1.320217 & -0.534979\end{array}$

$\begin{array}{llll}6 & -3.435166 & -1.028612 & 0.114871\end{array}$

$\begin{array}{lllll}6 & -5.544513 & 0.177036 & 0.290458\end{array}$

$\begin{array}{lllll}6 & -4.917437 & 1.336817 & -0.205153\end{array}$

$\begin{array}{llll}6 & -2.770187 & 0.136141 & -0.388612\end{array}$

$\begin{array}{llll}1 & -2.874030 & -1.957621 & 0.244726\end{array}$

$\begin{array}{llll}1 & -6.606339 & 0.190424 & 0.550367\end{array}$

$\begin{array}{lllll}1 & -5.494200 & 2.258092 & -0.330840\end{array}$

$\begin{array}{lllll}6 & -1.391377 & 0.169270 & -0.738124\end{array}$

$\begin{array}{lllll}1 & -3.085346 & 2.226670 & -0.918354\end{array}$

$\begin{array}{lllll}6 & -0.417815 & -0.945409 & -0.611413\end{array}$

$\begin{array}{lllll}1 & -0.995575 & 1.136728 & -1.068027\end{array}$

$\begin{array}{lllll}1 & 6.559436 & 0.177668 & -0.728083\end{array}$

$\begin{array}{lllll}6 & 0.439848 & -0.938469 & 0.740761\end{array}$ $\begin{array}{llll}6 & 1.425216 & 0.168261 & 0.850872\end{array}$

$\begin{array}{llll}1 & 1.056117 & 1.128488 & 1.228870\end{array}$

$\begin{array}{lllll}1 & 5.183545 & -1.897305 & -0.990289\end{array}$

$\begin{array}{llll}6 & 2.782101 & 0.134438 & 0.423942\end{array}$

$\begin{array}{lllll}6 & 5.513738 & 0.167270 & -0.409315\end{array}$

$\begin{array}{lllll}6 & 3.404210 & -1.022176 & -0.148309\end{array}$

$\begin{array}{llll}6 & 3.596735 & 1.306279 & 0.557497\end{array}$

$\begin{array}{llll}6 & 4.928553 & 1.318894 & 0.152251\end{array}$

$\begin{array}{lllll}6 & 4.737554 & -0.997928 & -0.554723\end{array}$

$1 \quad 2.825836 \quad-1.941644 \quad-0.270816$

$\begin{array}{llll}1 & 3.149471 & 2.206310 & 0.991844\end{array}$

$\begin{array}{llll}1 & 5.521845 & 2.230661 & 0.270085\end{array}$

$\begin{array}{llll}1 & -0.278595 & -0.897584 & 1.576491\end{array}$

$\begin{array}{llll}1 & 0.940241 & -1.920622 & 0.791338\end{array}$

$\begin{array}{lllll}1 & 0.304877 & -0.920111 & -1.444833\end{array}$

$\begin{array}{lllll}1 & -0.925099 & -1.923984 & -0.651996\end{array}$

16b)TS2x

UBPW91/6-31G*

$\begin{array}{llll}1 & 4.458203 & 0.586685 & 0.173012\end{array}$

$\begin{array}{lllll}6 & 3.612011 & -0.081748 & -0.016739\end{array}$

$\begin{array}{lllll}6 & 1.419893 & -1.790510 & -0.478876\end{array}$

$\begin{array}{lllll}6 & 2.348929 & 0.499031 & -0.387050\end{array}$

$\begin{array}{llll}6 & 3.754222 & -1.449277 & 0.132028\end{array}$

$\begin{array}{lllll}6 & 2.648711 & -2.316463 & -0.086090\end{array}$

$\begin{array}{lllll}6 & 1.251073 & -0.398852 & -0.634701\end{array}$

$\begin{array}{lllll}6 & 2.155084 & 1.892123 & -0.392464\end{array}$

$\begin{array}{lllll}1 & 4.720916 & -1.868848 & 0.426257\end{array}$

$\begin{array}{llll}1 & 2.774515 & -3.396615 & 0.031182\end{array}$

$1 \quad 0.577876 \quad-2.456300 \quad-0.689378$

$\begin{array}{lllll}6 & 0.822495 & 2.473653 & -0.400361\end{array}$

$\begin{array}{lllll}1 & 3.003074 & 2.531064 & -0.121553\end{array}$

$\begin{array}{lllll}1 & 0.845994 & 3.572843 & -0.440090\end{array}$

$\begin{array}{lllll}1 & 0.166207 & 2.109514 & -1.209233\end{array}$

$\begin{array}{llll}1 & -0.868888 & 2.779830 & 0.960824\end{array}$

$\begin{array}{llll}6 & -0.022911 & 2.074814 & 0.994584\end{array}$

$\begin{array}{llll}6 & -0.478689 & 0.691841 & 1.112836\end{array}$

$\begin{array}{llll}1 & 0.632004 & 2.363919 & 1.827139\end{array}$

$\begin{array}{llll}6 & -1.705613 & 0.185243 & 0.554081\end{array}$

$\begin{array}{lllll}6 & -2.528194 & 0.940223 & -0.333561\end{array}$

$\begin{array}{lllll}6 & -3.702471 & 0.401811 & -0.867368\end{array}$

$\begin{array}{lllll}6 & -4.110132 & -0.900131 & -0.533294\end{array}$

$\begin{array}{llll}6 & -3.318641 & -1.663123 & 0.345324\end{array}$

$\begin{array}{lllll}6 & -2.140435 & -1.135949 & 0.872961\end{array}$

$\begin{array}{lllll}1 & -2.246142 & 1.963336 & -0.597147\end{array}$

$\begin{array}{lllll}1 & -4.311296 & 1.006717 & -1.546327\end{array}$

$\begin{array}{lllll}1 & -5.033469 & -1.314147 & -0.947357\end{array}$

$\begin{array}{llll}1 & -3.626813 & -2.677310 & 0.617216\end{array}$

$\begin{array}{llll}1 & -1.528382 & -1.739250 & 1.551057\end{array}$

$\begin{array}{llll}1 & 0.051822 & 0.031940 & 1.803146\end{array}$

$1 \quad 0.352386-0.029493 \quad-1.126609$

17b) $\underline{T S 2 n}$

UBPW91/6-31G*

$\begin{array}{llll}1 & -2.220812 & 1.196061 & 2.308021\end{array}$

$\begin{array}{llll}6 & -1.951832 & 1.327581 & 1.254886\end{array}$

$\begin{array}{llll}6 & -1.239766 & 1.647871 & -1.449254\end{array}$

$\begin{array}{llll}6 & -2.024357 & 0.188215 & 0.382369\end{array}$

$\begin{array}{llll}6 & -1.517727 & 2.557392 & 0.793187\end{array}$

$\begin{array}{lllll}6 & -1.141474 & 2.723122 & -0.565829\end{array}$ $\begin{array}{llll}6 & -1.674905 & 0.389455 & -0.997747\end{array}$

$\begin{array}{llll}6 & -2.337163 & -1.096081 & 0.871777\end{array}$

$\begin{array}{llll}1 & -1.456017 & 3.407393 & 1.479208\end{array}$

$\begin{array}{lllll}1 & -0.800413 & 3.699481 & -0.921120\end{array}$

$\begin{array}{lllll}1 & -0.990135 & 1.784577 & -2.505684\end{array}$

$\begin{array}{llll}6 & -2.149741 & -2.282648 & 0.050776\end{array}$

$\begin{array}{llll}1 & -2.510336 & -1.214559 & 1.946783\end{array}$

$\begin{array}{llll}1 & -2.546850 & -3.192017 & 0.525949\end{array}$

$\begin{array}{lllll}1 & -2.596382 & -2.199194 & -0.953497\end{array}$

$1-0.619639-3.538795 \quad-0.860308$

$\begin{array}{lllll}6 & -0.538545 & -2.622337 & -0.253103\end{array}$

$\begin{array}{lllll}6 & 0.264367 & -1.623572 & -0.956225\end{array}$

$1 \quad-0.111781 \quad-2.898246 \quad 0.721171$

$\begin{array}{lllll}6 & 1.329473 & -0.852195 & -0.378351\end{array}$

$\begin{array}{llll}6 & 1.615139 & -0.835294 & 1.019413\end{array}$

$\begin{array}{lllll}6 & 2.678284 & -0.089183 & 1.530842\end{array}$

$\begin{array}{llll}6 & 3.508083 & 0.658038 & 0.677010\end{array}$

$\begin{array}{lllll}6 & 3.247514 & 0.657814 & -0.705335\end{array}$

$\begin{array}{lllll}6 & 2.179360 & -0.074390 & -1.221473\end{array}$

$1 \quad 0.988134-1.407696 \quad 1.707382$

$\begin{array}{llll}1 & 2.869017 & -0.094255 & 2.608466\end{array}$

$\begin{array}{llll}1 & 4.346845 & 1.230675 & 1.082177\end{array}$

$1 \quad 3.884199 \quad 1.236056-1.381940$

$\begin{array}{lllll}1 & 1.983753 & -0.065534 & -2.298717\end{array}$

$\begin{array}{lllll}1 & -1.885335 & -0.392324 & -1.727373\end{array}$

$\begin{array}{llll}1 & 0.185495 & -1.594356 & -2.048176\end{array}$

\section{8b)TScisDCB}

UBPW91/6-31G*

$6 \quad 4.175360 \quad-1.588145-0.162235$

$\begin{array}{lllll}6 & -1.478684 & 1.513754 & 0.800620\end{array}$

$\begin{array}{llll}6 & -0.954353 & 2.577128 & -0.136356\end{array}$

$\begin{array}{llll}6 & 1.202033 & 1.453285 & 0.266604\end{array}$

$\begin{array}{llll}6 & 0.324261 & 2.034299 & -0.810273\end{array}$

$\begin{array}{lllll}1 & 0.027973 & 1.246283 & -1.524878\end{array}$

$\begin{array}{lllll}1 & 0.849138 & 2.811173 & -1.409670\end{array}$

$\begin{array}{llll}1 & -1.317739 & 1.652267 & 1.873929\end{array}$

$\begin{array}{llll}1 & 1.179624 & 1.956831 & 1.239190\end{array}$

$\begin{array}{llll}6 & 2.184621 & 0.437547 & 0.097267\end{array}$

$\begin{array}{llll}1 & -0.711777 & 3.485477 & 0.441948\end{array}$

$\begin{array}{lllll}1 & -1.703684 & 2.878414 & -0.891715\end{array}$

$\begin{array}{llll}1 & 1.802399 & 0.031588 & -2.016840\end{array}$

$\begin{array}{lllll}6 & 2.406500 & -0.234975 & -1.146099\end{array}$

$\begin{array}{llll}6 & 3.005868 & 0.046602 & 1.203413\end{array}$

$\begin{array}{llll}1 & 4.586204 & -1.221128 & 1.939168\end{array}$

$\begin{array}{llll}6 & 3.975604 & -0.944102 & 1.074515\end{array}$

$6 \quad 3.383823 \quad-1.222480-1.266604$

$1 \quad 4.937376 \quad-2.365739-0.262747$

$1 \quad 3.533166-1.717943 \quad-2.230742$

$\begin{array}{llll}1 & 2.856660 & 0.543568 & 2.167531\end{array}$

$\begin{array}{llll}6 & -2.247028 & 0.382595 & 0.405623\end{array}$

$\begin{array}{lllll}6 & -3.783193 & -1.911715 & -0.313144\end{array}$

$\begin{array}{llll}6 & -2.669254 & -0.574540 & 1.383749\end{array}$

$\begin{array}{lllll}6 & -2.639625 & 0.141117 & -0.949752\end{array}$

$\begin{array}{lllll}6 & -3.389247 & -0.984029 & -1.294167\end{array}$

$\begin{array}{llll}6 & -3.417226 & -1.693784 & 1.030011\end{array}$

$\begin{array}{llll}1 & -2.385797 & -0.414124 & 2.428930\end{array}$

$\begin{array}{llll}1 & -2.359530 & 0.857240 & -1.726605\end{array}$

$\begin{array}{llll}1 & -3.676431 & -1.141148 & -2.338351\end{array}$

$\begin{array}{llll}1 & -3.722511 & -2.407428 & 1.801147\end{array}$

$1-4.371826 \quad-2.790671-0.589323$ 
19b)TStransDCB

UBPW91/6-31G*

$\begin{array}{llll}6 & -4.859803 & -1.181091 & 1.032547\end{array}$

$\begin{array}{llll}6 & 1.363075 & 0.835805 & 0.374952\end{array}$

$\begin{array}{lllll}6 & 0.749783 & 2.094515 & -0.170277\end{array}$

$\begin{array}{lllll}6 & -1.363230 & 0.835925 & -0.375167\end{array}$

$\begin{array}{llll}6 & -0.749830 & 2.094581 & 0.170063\end{array}$

$\begin{array}{llll}1 & -0.865956 & 2.142822 & 1.269190\end{array}$

$\begin{array}{llll}1 & -1.248733 & 3.005357 & -0.224679\end{array}$

$\begin{array}{lllll}6 & 2.530758 & 0.185045 & -0.112145\end{array}$

$\begin{array}{lllll}1 & -0.913030 & 0.432438 & -1.288924\end{array}$

$\begin{array}{llll}6 & -2.530821 & 0.185111 & 0.112079\end{array}$

$\begin{array}{llll}1 & 0.865912 & 2.142740 & -1.269406\end{array}$

$\begin{array}{llll}1 & 1.248772 & 3.005251 & 0.224454\end{array}$

$\begin{array}{llll}1 & -2.952627 & 1.564330 & 1.756051\end{array}$

$\begin{array}{llll}6 & -3.276519 & 0.657240 & 1.239340\end{array}$

$\begin{array}{lllll}6 & -3.009122 & -1.001368 & -0.532758\end{array}$

$\begin{array}{lllll}1 & -4.482997 & -2.572448 & -0.592691\end{array}$

$\begin{array}{llll}6 & -4.144653 & -1.667278 & -0.079518\end{array}$

$\begin{array}{lllll}6 & -4.415171 & -0.014845 & 1.682568\end{array}$

$\begin{array}{lllll}1 & -5.752362 & -1.703937 & 1.386554\end{array}$

$\begin{array}{llll}1 & -4.967337 & 0.371352 & 2.544792\end{array}$

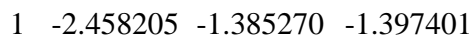

$\begin{array}{llll}1 & 0.912710 & 0.432231 & 1.288589\end{array}$

$\begin{array}{llll}6 & 4.859934 & -1.181024 & -1.032311\end{array}$

$\begin{array}{llll}6 & 3.276671 & 0.657314 & -1.239205\end{array}$

$\begin{array}{lllll}6 & 3.008937 & -1.001507 & 0.532644\end{array}$

$\begin{array}{llll}6 & 4.144565 & -1.667354 & 0.079551\end{array}$

$\begin{array}{lllll}6 & 4.415421 & -0.014706 & -1.682283\end{array}$

$\begin{array}{lllll}1 & 2.952869 & 1.564461 & -1.755871\end{array}$

$\begin{array}{llll}1 & 2.457851 & -1.385517 & 1.397131\end{array}$

$\begin{array}{lllll}1 & 4.482814 & -2.572582 & 0.592683\end{array}$

$\begin{array}{lllll}1 & 4.967756 & 0.371600 & -2.544350\end{array}$

$\begin{array}{lllll}1 & 5.752569 & -1.703819 & -1.386201\end{array}$

\section{0b)TStransDCB'}

UBPW91/6-31G*

$\begin{array}{llll}6 & -0.943937 & 0.476791 & 0.951571\end{array}$

$\begin{array}{llll}6 & -0.335567 & 1.842382 & 0.698661\end{array}$

$\begin{array}{lllll}6 & 0.943930 & 0.476783 & -0.951555\end{array}$

$\begin{array}{lllll}6 & 0.335564 & 1.842377 & -0.698654\end{array}$

$\begin{array}{lllll}1 & -0.426919 & 2.031987 & -1.472636\end{array}$

$\begin{array}{llll}1 & 1.080264 & 2.656232 & -0.784455\end{array}$

$\begin{array}{llll}1 & 0.426916 & 2.031995 & 1.472641\end{array}$

$\begin{array}{llll}1 & -1.080266 & 2.656239 & 0.784458\end{array}$

$\begin{array}{llll}1 & -0.376459 & -0.212875 & 1.583258\end{array}$

$\begin{array}{llll}1 & 0.376444 & -0.212890 & -1.583227\end{array}$

$\begin{array}{llll}6 & -2.223977 & 0.043385 & 0.506496\end{array}$

$\begin{array}{lllll}6 & -3.106396 & 0.869966 & -0.260583\end{array}$

$\begin{array}{lllll}6 & -4.356983 & 0.406808 & -0.668125\end{array}$

$\begin{array}{lllll}6 & -4.784870 & -0.892069 & -0.335806\end{array}$

$\begin{array}{lllll}6 & -3.936882 & -1.726257 & 0.419252\end{array}$

$\begin{array}{lllll}6 & -2.687175 & -1.272598 & 0.832443\end{array}$

$\begin{array}{llll}1 & -2.796293 & 1.883910 & -0.526953\end{array}$

$\begin{array}{llll}1 & -5.010829 & 1.062405 & -1.251359\end{array}$

$1-5.766263-1.249592 \quad-0.658832$

$\begin{array}{llll}1 & -4.261685 & -2.736660 & 0.685446\end{array}$

$\begin{array}{llll}1 & -2.034702 & -1.925775 & 1.420744\end{array}$

$\begin{array}{lllll}6 & 2.223974 & 0.043380 & -0.506489\end{array}$ $\begin{array}{llll}6 & 3.106404 & 0.869969 & 0.260569\end{array}$

$\begin{array}{lllll}6 & 4.356996 & 0.406813 & 0.668100\end{array}$

$\begin{array}{lllll}6 & 4.784876 & -0.892068 & 0.335790\end{array}$

$\begin{array}{lllll}6 & 3.936877 & -1.726264 & -0.419245\end{array}$

$\begin{array}{lllll}6 & 2.687166 & -1.272607 & -0.832426\end{array}$

$\begin{array}{llll}1 & 2.796306 & 1.883917 & 0.526932\end{array}$

$\begin{array}{llll}1 & 5.010851 & 1.062416 & 1.251318\end{array}$

$\begin{array}{lllll}1 & 5.766273 & -1.249589 & 0.658808\end{array}$

$14.261675 \quad-2.736671 \quad-0.685432$

$1 \quad 2.034683 \quad-1.925790 \quad-1.420711$

21b) $\underline{A H}$

RBPW91/6-31G*

$\begin{array}{llll}6 & -3.493743 & -0.624665 & 0.201633\end{array}$

$\begin{array}{llll}6 & -2.384978 & 0.322456 & 0.143366\end{array}$

$\begin{array}{lllll}6 & 0.085968 & 0.685698 & -0.377818\end{array}$

$\begin{array}{lllll}6 & -1.522112 & 2.678374 & -0.229647\end{array}$

$\begin{array}{llll}6 & -0.121725 & 2.138241 & 0.116176\end{array}$

$\begin{array}{lllll}6 & -2.605434 & 1.649761 & -0.054394\end{array}$

$\begin{array}{llll}6 & -0.967928 & -0.242575 & 0.300573\end{array}$

$\begin{array}{lllll}6 & 1.521783 & 0.225071 & -0.162252\end{array}$

$\begin{array}{llll}1 & -1.740848 & 3.572144 & 0.385765\end{array}$

$\begin{array}{llll}1 & 0.656166 & 2.792291 & -0.312825\end{array}$

$\begin{array}{llll}6 & -3.294844 & -1.965526 & 0.062654\end{array}$

$\begin{array}{llll}1 & -0.737137 & -0.230575 & 1.391627\end{array}$

$\begin{array}{llll}1 & -1.849414 & -3.559104 & -0.419777\end{array}$

$\begin{array}{lllll}1 & -0.117119 & 0.662942 & -1.464957\end{array}$

$\begin{array}{lllll}1 & -1.541147 & 3.044621 & -1.277424\end{array}$

$\begin{array}{llll}1 & 0.020638 & 2.162540 & 1.211774\end{array}$

$\begin{array}{lllll}1 & -3.642290 & 2.000745 & -0.133573\end{array}$

$\begin{array}{lllll}6 & -0.866775 & -1.697036 & -0.115579\end{array}$

$\begin{array}{lllll}1 & -4.145752 & -2.653079 & 0.068805\end{array}$

$\begin{array}{lllll}1 & -4.507481 & -0.218601 & 0.287784\end{array}$

$\begin{array}{lllll}6 & -1.956728 & -2.497082 & -0.177194\end{array}$

$\begin{array}{lllll}1 & 0.135683 & -2.098492 & -0.290830\end{array}$

$\begin{array}{lllll}6 & 4.215331 & -0.582609 & 0.208109\end{array}$

$\begin{array}{llll}6 & 2.078023 & 0.122127 & 1.130238\end{array}$

$\begin{array}{lllll}6 & 2.344527 & -0.088784 & -1.261819\end{array}$

$\begin{array}{lllll}6 & 3.677440 & -0.487540 & -1.082912\end{array}$

$\begin{array}{llll}6 & 3.408800 & -0.276943 & 1.314805\end{array}$

$\begin{array}{llll}1 & 1.465439 & 0.356149 & 2.006838\end{array}$

$\begin{array}{lllll}1 & 1.931948 & -0.017364 & -2.273801\end{array}$

$\begin{array}{llll}1 & 4.294548 & -0.725075 & -1.954570\end{array}$

$\begin{array}{llll}1 & 3.817174 & -0.349283 & 2.327289\end{array}$

$\begin{array}{llll}1 & 5.253969 & -0.893654 & 0.352256\end{array}$

22b) $\underline{A H}$

RBPW91/6-31G*

$\begin{array}{llll}6 & -2.973274 & -0.371577 & -0.862311\end{array}$

$\begin{array}{lllll}6 & -1.912988 & 0.524314 & -0.412997\end{array}$

$\begin{array}{llll}6 & 0.158460 & 0.823541 & 1.091359\end{array}$

$\begin{array}{lllll}6 & -0.374587 & 2.499861 & -0.788670\end{array}$

$\begin{array}{llll}6 & 0.089858 & 2.317099 & 0.667485\end{array}$

$\begin{array}{llll}6 & -1.533507 & 1.607171 & -1.143874\end{array}$

$\begin{array}{llll}6 & -1.257084 & 0.201796 & 0.935073\end{array}$

$\begin{array}{llll}1 & 0.392051 & 0.809186 & 2.173050\end{array}$

$\begin{array}{llll}1 & -0.641106 & 3.559315 & -0.963036\end{array}$

$\begin{array}{llll}1 & 1.072838 & 2.795153 & 0.816349\end{array}$

$\begin{array}{llll}6 & -3.157896 & -1.599748 & -0.298977\end{array}$

$\begin{array}{llll}1 & -1.878821 & 0.725637 & 1.699049\end{array}$
$1-2.368858$-3.117868 1.093328

$\begin{array}{llll}6 & 1.302798 & 0.085697 & 0.396718\end{array}$

$\begin{array}{llll}1 & 0.464442 & 2.293912 & -1.484431\end{array}$

$\begin{array}{llll}1 & -0.621067 & 2.830144 & 1.340825\end{array}$

$\begin{array}{lllll}1 & -2.065904 & 1.822791 & -2.078999\end{array}$

$\begin{array}{llll}6 & -1.343915 & -1.264497 & 1.309530\end{array}$

$\begin{array}{lllll}1 & -3.936058 & -2.267570 & -0.680256\end{array}$

$1 \quad-3.578666 \quad-0.057813-1.719767$

$\begin{array}{llll}6 & -2.275477 & -2.079131 & 0.761557\end{array}$

$1 \quad-0.674871 \quad-1.622349 \quad 2.099016$

$\begin{array}{llll}6 & 3.532918 & -1.178502 & -0.822325\end{array}$

$\begin{array}{lllll}6 & 1.140696 & -0.724540 & -0.744313\end{array}$

$\begin{array}{llll}6 & 2.606492 & 0.241902 & 0.914913\end{array}$

$\begin{array}{lllll}6 & 3.710564 & -0.377546 & 0.315957\end{array}$

$\begin{array}{llll}6 & 2.244529 & -1.349850 & -1.345757\end{array}$

$\begin{array}{lllll}1 & 0.145699 & -0.876754 & -1.166856\end{array}$

$\begin{array}{llll}1 & 2.754730 & 0.857552 & 1.809400\end{array}$

$\begin{array}{llll}1 & 4.708650 & -0.241317 & 0.743220\end{array}$

$\begin{array}{llll}1 & 2.090860 & -1.977242 & -2.228968\end{array}$

$14.390554-1.668721-1.292133$

\section{3b) BHx \\ RBPW91/6-31G*}

$\begin{array}{llll}6 & -3.083149 & 0.292895 & 1.155335\end{array}$

$\begin{array}{llll}6 & -1.840504 & 0.602055 & 0.459070\end{array}$

$\begin{array}{lllll}6 & -0.427083 & 0.183079 & -1.590273\end{array}$

$\begin{array}{lllll}6 & 0.000375 & 2.247117 & -0.159354\end{array}$

$\begin{array}{lllll}6 & 0.659025 & 1.141759 & -1.027623\end{array}$

$\begin{array}{llll}6 & -1.214923 & 1.802156 & 0.610562\end{array}$

$\begin{array}{lllll}6 & -1.260431 & -0.467556 & -0.466534\end{array}$

$\begin{array}{lllll}1 & 0.052144 & -0.598880 & -2.205141\end{array}$

$\begin{array}{llll}1 & 0.754033 & 2.676328 & 0.528236\end{array}$

$\begin{array}{lllll}1 & 1.127665 & 1.634757 & -1.898523\end{array}$

$\begin{array}{llll}6 & -3.865463 & -0.758900 & 0.779595\end{array}$

$\begin{array}{llll}1 & -0.548103 & -1.074395 & 0.141069\end{array}$

$\begin{array}{lllll}1 & -4.202745 & -2.359153 & -0.699151\end{array}$

$\begin{array}{lllll}1 & -1.101687 & 0.753805 & -2.253689\end{array}$

$\begin{array}{lllll}1 & -0.294924 & 3.091036 & -0.815981\end{array}$

$\begin{array}{lllll}6 & 1.768097 & 0.373810 & -0.313643\end{array}$

$\begin{array}{llll}1 & -1.646192 & 2.533634 & 1.306237\end{array}$

$\begin{array}{lllll}6 & -2.297377 & -1.448242 & -0.968981\end{array}$

$\begin{array}{lllll}1 & -4.813617 & -0.949832 & 1.290900\end{array}$

$\begin{array}{llll}1 & -3.410625 & 0.973758 & 1.948657\end{array}$

$\begin{array}{lllll}6 & -3.492653 & -1.601980 & -0.352193\end{array}$

$\begin{array}{lllll}1 & -2.014251 & -2.086485 & -1.813381\end{array}$

$\begin{array}{llll}6 & 3.839814 & -1.113383 & 0.928455\end{array}$

$\begin{array}{lllll}6 & 2.879179 & -0.084380 & -1.051647\end{array}$

$\begin{array}{llll}6 & 1.719805 & 0.073901 & 1.063227\end{array}$

$\begin{array}{llll}6 & 2.744117 & -0.663081 & 1.677677\end{array}$

$\begin{array}{llll}6 & 3.904231 & -0.819920 & -0.442217\end{array}$

$\begin{array}{lllll}1 & 2.941516 & 0.145195 & -2.121176\end{array}$

$\begin{array}{llll}1 & 0.875749 & 0.427616 & 1.663258\end{array}$

$\begin{array}{llll}1 & 2.684314 & -0.881698 & 2.748085\end{array}$

$14.758147 \quad-1.158139-1.036881$

$14.640215 \quad-1.683517 \quad 1.408739$

\section{4b) $\underline{B H n}$}

RBPW91/6-31G*

$\begin{array}{llll}6 & 3.744872 & 0.988278 & 0.073862\end{array}$

$\begin{array}{lllll}6 & 2.305810 & 0.805211 & -0.076396\end{array}$ 
$\begin{array}{llll}6 & 0.361956 & -0.817247 & -0.000530\end{array}$

$\begin{array}{llll}6 & -0.070534 & 1.685297 & 0.116604\end{array}$

$\begin{array}{llll}6 & -0.555907 & 0.335113 & -0.474315\end{array}$

$\begin{array}{lllll}6 & 1.429294 & 1.821474 & 0.143839\end{array}$

$\begin{array}{lllll}6 & 1.810662 & -0.586344 & -0.479930\end{array}$

$\begin{array}{lllll}1 & -0.016265 & -1.779988 & -0.386658\end{array}$

$\begin{array}{lllll}1 & -0.523967 & 2.518554 & -0.453528\end{array}$

$\begin{array}{lllll}6 & -2.029213 & 0.075344 & -0.189785\end{array}$

$\begin{array}{llll}6 & 4.588403 & -0.077112 & 0.182603\end{array}$

$1 \quad 1.780705-0.605459-1.595514$

$\begin{array}{lllll}1 & 4.759704 & -2.265098 & 0.400132\end{array}$

$\begin{array}{llll}1 & 0.350573 & -0.884859 & 1.102112\end{array}$

$\begin{array}{llll}1 & -0.461619 & 1.805750 & 1.147756\end{array}$

$\begin{array}{lllll}1 & -0.440140 & 0.400586 & -1.572988\end{array}$

$\begin{array}{llll}1 & 1.828131 & 2.811662 & 0.399263\end{array}$

$\begin{array}{lllll}6 & 2.768185 & -1.693017 & -0.086674\end{array}$

$\begin{array}{llll}1 & 5.659807 & 0.081768 & 0.336205\end{array}$

$\begin{array}{llll}1 & 4.125408 & 2.010766 & 0.172204\end{array}$

$\begin{array}{lllll}6 & 4.071935 & -1.443422 & 0.176748\end{array}$

$\begin{array}{lllll}1 & 2.380878 & -2.717874 & -0.095541\end{array}$

$\begin{array}{llll}6 & -4.775380 & -0.416887 & 0.314981\end{array}$

$\begin{array}{llll}6 & -2.503292 & -0.109588 & 1.125743\end{array}$

$\begin{array}{lllll}6 & -2.960571 & 0.008521 & -1.243581\end{array}$

$\begin{array}{llll}6 & -4.320189 & -0.234415 & -0.998157\end{array}$

$\begin{array}{llll}6 & -3.860098 & -0.353943 & 1.376890\end{array}$

$\begin{array}{llll}1 & -1.803629 & -0.064709 & 1.966477\end{array}$

$1 \quad-2.613486 \quad 0.149149 \quad-2.272923$

$\begin{array}{lllll}1 & -5.023238 & -0.281019 & -1.835186\end{array}$

$\begin{array}{llll}1 & -4.203900 & -0.495991 & 2.405872\end{array}$

$\begin{array}{llll}1 & -5.834568 & -0.606812 & 0.510909\end{array}$

25b) cis- $D C B$

RBPW91/6-31G*

$\begin{array}{lllll}6 & 3.282181 & -1.701604 & -0.377877\end{array}$

$\begin{array}{llll}6 & -0.833368 & 1.557740 & 0.806672\end{array}$

$\begin{array}{lllll}6 & -1.166958 & 2.824363 & -0.039945\end{array}$

$\begin{array}{llll}6 & 0.673453 & 1.711120 & 0.275965\end{array}$

$\begin{array}{llll}6 & 0.117103 & 2.593467 & -0.879625\end{array}$

$\begin{array}{lllll}1 & -0.090397 & 2.000254 & -1.784240\end{array}$

$1 \quad 0.717492 \quad 3.470276-1.170923$

$\begin{array}{llll}1 & -0.902154 & 1.700461 & 1.898020\end{array}$

$\begin{array}{llll}1 & 1.179794 & 2.372915 & 1.000088\end{array}$

$\begin{array}{llll}6 & 1.565433 & 0.520962 & 0.024606\end{array}$

$\begin{array}{llll}1 & -1.092616 & 3.734429 & 0.576404\end{array}$

$\begin{array}{lllll}1 & -2.134178 & 2.846639 & -0.565644\end{array}$

$\begin{array}{lllll}1 & 0.559423 & -0.175676 & -1.768597\end{array}$

$\begin{array}{lllll}6 & 1.379795 & -0.350600 & -1.068372\end{array}$

$\begin{array}{llll}6 & 2.630618 & 0.251159 & 0.909223\end{array}$

$1 \quad 4.301624-1.028487 \quad 1.415166$

$\begin{array}{lllll}6 & 3.480536 & -0.846031 & 0.715334\end{array}$

$\begin{array}{lllll}6 & 2.227975 & -1.448443 & -1.267884\end{array}$

$1 \quad 3.944886-2.557232-0.536921$

$\begin{array}{lllll}1 & 2.063054 & -2.109520 & -2.123968\end{array}$

$\begin{array}{llll}1 & 2.797038 & 0.918423 & 1.762082\end{array}$

$\begin{array}{llll}6 & -1.550323 & 0.270690 & 0.455739\end{array}$

$\begin{array}{lllll}6 & -2.847730 & -2.193280 & -0.102684\end{array}$

$\begin{array}{llll}6 & -1.508695 & -0.802339 & 1.371641\end{array}$

$\begin{array}{lllll}6 & -2.260515 & 0.078200 & -0.746789\end{array}$

$\begin{array}{lllll}6 & -2.901364 & -1.138926 & -1.024183\end{array}$

$\begin{array}{llll}6 & -2.146687 & -2.018853 & 1.100136\end{array}$

$\begin{array}{lllll}1 & -0.966449 & -0.675819 & 2.314352\end{array}$
$1 \quad-2.325801 \quad 0.887608 \quad-1.478953$

$\begin{array}{lllll}1 & -3.449473 & -1.258547 & -1.963653\end{array}$

$\begin{array}{llll}1 & -2.099730 & -2.831070 & 1.831627\end{array}$

$\begin{array}{lllll}1 & -3.351232 & -3.140405 & -0.316588\end{array}$

26b)trans-DCB

RBPW91/6-31G*

$\begin{array}{llll}6 & 0.839693 & -3.855028 & -1.576870\end{array}$

$\begin{array}{llll}6 & 0.390859 & 0.688185 & 1.130976\end{array}$

$\begin{array}{llll}6 & 0.124863 & 0.765814 & 2.667988\end{array}$

$\begin{array}{llll}6 & -0.390859 & -0.688185 & 1.130976\end{array}$

$\begin{array}{llll}6 & -0.124863 & -0.765814 & 2.667988\end{array}$

$\begin{array}{lllll}1 & 0.795640 & -1.328576 & 2.891382\end{array}$

$\begin{array}{llll}1 & -0.937151 & -1.163354 & 3.296602\end{array}$

$\begin{array}{llll}6 & -0.041723 & 1.795486 & 0.201485\end{array}$

$\begin{array}{lllll}1 & -1.460473 & -0.472066 & 0.964913\end{array}$

$\begin{array}{llll}6 & 0.041723 & -1.795486 & 0.201485\end{array}$

$\begin{array}{llll}1 & -0.795640 & 1.328576 & 2.891382\end{array}$

$\begin{array}{llll}1 & 0.937151 & 1.163354 & 3.296602\end{array}$

$\begin{array}{llll}1 & 2.044056 & -1.992001 & 1.022383\end{array}$

$\begin{array}{llll}6 & 1.334803 & -2.355039 & 0.271436\end{array}$

$\begin{array}{lllll}6 & -0.839693 & -2.290235 & -0.780379\end{array}$

$\begin{array}{lllll}1 & -1.152704 & -3.675095 & -2.414289\end{array}$

$\begin{array}{lllll}6 & -0.448578 & -3.308523 & -1.661370\end{array}$

$\begin{array}{lllll}6 & 1.730889 & -3.372973 & -0.605805\end{array}$

$\begin{array}{lllll}1 & 1.148012 & -4.650395 & -2.261478\end{array}$

$1 \quad 2.738776-3.792175 \quad-0.530409$

$\begin{array}{lllll}1 & -1.847236 & -1.867743 & -0.853879\end{array}$

$\begin{array}{lllll}1 & 1.460473 & 0.472066 & 0.964913\end{array}$

$\begin{array}{llll}6 & -0.839693 & 3.855028 & -1.576870\end{array}$

$\begin{array}{llll}6 & -1.334803 & 2.355039 & 0.271436\end{array}$

$\begin{array}{lllll}6 & 0.839693 & 2.290235 & -0.780379\end{array}$

$\begin{array}{lllll}6 & 0.448578 & 3.308523 & -1.661370\end{array}$

$\begin{array}{lllll}6 & -1.730889 & 3.372973 & -0.605805\end{array}$

$\begin{array}{llll}1 & -2.044056 & 1.992001 & 1.022383\end{array}$

$\begin{array}{lllll}1 & 1.847236 & 1.867743 & -0.853879\end{array}$

$\begin{array}{lllll}1 & 1.152704 & 3.675095 & -2.414289\end{array}$

$1-2.738776 \quad 3.792175 \quad-0.530409$

$\begin{array}{lllll}1 & -1.148012 & 4.650395 & -2.261478\end{array}$

\section{7b) $\underline{A H x}+\mathrm{M}$ : TS Habs}

UBPW91/6-31G*

$\begin{array}{llll}6 & 1.329735 & 2.019293 & 1.196589\end{array}$

$\begin{array}{llll}6 & 0.085298 & 2.058192 & 0.442944\end{array}$

$\begin{array}{llll}6 & -2.236747 & 1.089897 & 0.021094\end{array}$

$\begin{array}{lllll}6 & -1.523659 & 3.254146 & -1.114887\end{array}$

$\begin{array}{lllll}6 & -2.206746 & 1.887554 & -1.303791\end{array}$

$\begin{array}{llll}6 & -0.250791 & 3.147975 & -0.322165\end{array}$

$\begin{array}{lllll}6 & -0.793246 & 0.856564 & 0.527204\end{array}$

$\begin{array}{lllll}6 & -3.107582 & -0.161429 & -0.035102\end{array}$

$\begin{array}{lllll}1 & -1.321042 & 3.711707 & -2.102030\end{array}$

$\begin{array}{llll}1 & -3.234131 & 2.020244 & -1.682747\end{array}$

$\begin{array}{llll}6 & 1.609184 & 1.000689 & 2.067248\end{array}$

$\begin{array}{llll}1 & -0.128114 & -0.008675 & -0.469554\end{array}$

$\begin{array}{llll}1 & 0.870212 & -0.825495 & 3.036730\end{array}$

$\begin{array}{llll}1 & -2.703589 & 1.756528 & 0.775940\end{array}$

$\begin{array}{llll}1 & -2.222798 & 3.957402 & -0.613631\end{array}$

$\begin{array}{llll}1 & -1.655119 & 1.315494 & -2.070223\end{array}$

$\begin{array}{lllll}1 & 0.438715 & 3.999908 & -0.350377\end{array}$

$\begin{array}{llll}6 & -0.544170 & -0.062342 & 1.614894\end{array}$ $\begin{array}{llll}1 & 2.551978 & 0.996145 & 2.621073\end{array}$

$\begin{array}{llll}1 & 2.026336 & 2.855630 & 1.078017\end{array}$

$\begin{array}{llll}6 & 0.655981 & -0.058646 & 2.287103\end{array}$

$\begin{array}{llll}1 & -1.272498 & -0.857436 & 1.797895\end{array}$

$\begin{array}{lllll}6 & -4.758583 & -2.471353 & -0.067673\end{array}$

$\begin{array}{lllll}6 & -3.190085 & -0.980043 & -1.179704\end{array}$

$\begin{array}{llll}6 & -3.883661 & -0.521082 & 1.086545\end{array}$

$\begin{array}{lllll}6 & -4.696940 & -1.662984 & 1.076472\end{array}$

$\begin{array}{llll}6 & -4.004530 & -2.122072 & -1.196943\end{array}$

$1-2.625703 \quad-0.718243-2.078428$

$\begin{array}{llll}1 & -3.849916 & 0.110011 & 1.981008\end{array}$

$\begin{array}{llll}1 & -5.288327 & -1.915944 & 1.961481\end{array}$

$1-4.052806 \quad-2.737816 \quad-2.100087$

$1 \quad-5.394606 \quad-3.361024 \quad-0.082069$

$\begin{array}{lllll}1 & -0.346570 & -1.339391 & -1.713591\end{array}$

$\begin{array}{lllll}1 & 0.838542 & 0.015118 & -2.054544\end{array}$

$\begin{array}{lllll}1 & 1.220171 & -2.495897 & -0.288389\end{array}$

$\begin{array}{lllll}6 & 2.910386 & -1.183711 & -0.549841\end{array}$

$6 \begin{array}{llll}6 & 0.515509 & -0.764767 & -1.349752\end{array}$

$\begin{array}{lllll}6 & 1.518366 & -1.530453 & -0.712888\end{array}$

$\begin{array}{lllll}6 & 5.684368 & -0.620655 & -0.245150\end{array}$

$\begin{array}{lllll}6 & 3.462457 & 0.057391 & -0.974905\end{array}$

$\begin{array}{llll}6 & 3.799133 & -2.125094 & 0.042321\end{array}$

$\begin{array}{llll}6 & 5.159558 & -1.850769 & 0.188983\end{array}$

$\begin{array}{lllll}6 & 4.824894 & 0.328053 & -0.824726\end{array}$

$\begin{array}{lllll}1 & 2.811570 & 0.812329 & -1.422014\end{array}$

$\begin{array}{llll}1 & 3.397807 & -3.085684 & 0.382030\end{array}$

$1 \quad 5.817721 \quad-2.597343 \quad 0.643644$

$\begin{array}{lllll}1 & 5.223227 & 1.289995 & -1.161856\end{array}$

$1 \quad 6.750168 \quad-0.403685-0.131500$

28b) $\underline{A H n}+\mathrm{M}$ : TS Habs

UBPW91/6-31G*

$\begin{array}{llll}6 & 0.710746 & 0.903265 & 1.757653\end{array}$

$\begin{array}{llll}6 & -0.296894 & 1.126620 & 0.730991\end{array}$

$\begin{array}{llll}6 & -1.895037 & 0.100317 & -1.017339\end{array}$

$\begin{array}{lllll}6 & -1.925611 & 2.622087 & -0.501101\end{array}$

$\begin{array}{lllll}6 & -1.946210 & 1.542250 & -1.597535\end{array}$

$\begin{array}{llll}6 & -0.846844 & 2.367811 & 0.514379\end{array}$

$\begin{array}{lllll}6 & -0.680861 & -0.051288 & -0.090110\end{array}$

$\begin{array}{lllll}1 & -1.747133 & -0.586282 & -1.872785\end{array}$

$\begin{array}{lllll}1 & -1.791191 & 3.618021 & -0.962283\end{array}$

$\begin{array}{llll}1 & -2.841834 & 1.653058 & -2.231625\end{array}$

$\begin{array}{llll}6 & 1.129459 & -0.358484 & 2.087409\end{array}$

$\begin{array}{lllll}1 & 0.433669 & -0.063532 & -1.040083\end{array}$

$\begin{array}{llll}1 & 0.852034 & -2.515777 & 1.777239\end{array}$

$\begin{array}{llll}6 & -3.228585 & -0.297555 & -0.376422\end{array}$

$\begin{array}{llll}1 & -2.914360 & 2.666294 & 0.002121\end{array}$

$\begin{array}{lllll}1 & -1.067235 & 1.682601 & -2.251665\end{array}$

$\begin{array}{llll}1 & -0.508072 & 3.208716 & 1.130779\end{array}$

$\begin{array}{llll}6 & -0.376999 & -1.359885 & 0.449618\end{array}$

$\begin{array}{llll}1 & 1.880847 & -0.498928 & 2.869430\end{array}$

$\begin{array}{lllll}1 & 1.092265 & 1.774087 & 2.300915\end{array}$

$\begin{array}{lllll}6 & 0.560936 & -1.516734 & 1.441539\end{array}$

$1 \quad-0.822030 \quad-2.235015 \quad-0.035597$

$\begin{array}{llll}6 & 4.991638 & 1.126113 & 0.405121\end{array}$

$\begin{array}{llll}1 & 5.122038 & 2.142381 & 0.789474\end{array}$

$\begin{array}{llll}1 & 6.900471 & 0.423753 & 1.170596\end{array}$

$\begin{array}{llll}6 & 5.991333 & 0.162321 & 0.621978\end{array}$

$14.513862 \quad-2.490389-0.952304$

$\begin{array}{llll}1 & 6.579867 & -1.901491 & 0.292310\end{array}$ 
$\begin{array}{rrrr}6 & 3.828554 & 0.800405 & -0.296398 \\ 6 & 4.648323 & -1.472807 & -0.570712 \\ 1 & 3.061288 & 1.563512 & -0.446586 \\ 6 & 5.809828 & -1.141610 & 0.128582 \\ 1 & 0.793308 & -0.419418 & -2.810821 \\ 1 & 1.574431 & 1.024100 & -2.003434 \\ 1 & 2.398361 & -1.953477 & -1.840752 \\ 6 & 3.625951 & -0.511488 & -0.810656 \\ 6 & 1.388100 & -0.058843 & -1.958738 \\ 6 & 2.458089 & -0.893461 & -1.567797 \\ 6 & -5.753681 & -1.002498 & 0.702923 \\ 6 & -3.526669 & -0.058840 & 0.979854 \\ 6 & -4.220158 & -0.899677 & -1.177907 \\ 6 & -5.469719 & -1.249484 & -0.648410 \\ 6 & -4.776733 & -0.406632 & 1.513217 \\ 1 & -2.769660 & 0.392168 & 1.626231 \\ 1 & -4.005612 & -1.101592 & -2.233379 \\ 1 & -6.219136 & -1.721909 & -1.290666 \\ 1 & -4.984051 & -0.214416 & 2.570257 \\ 1 & -6.725876 & -1.277899 & 1.121794\end{array}$

29b)AHx + M: TS Ene1 RBPW91/6-31G*

$\begin{array}{lccl}1 & -2.339377 & 1.660545 & 1.70778 \\ 6 & -1.377996 & 1.737064 & 1.191273 \\ 6 & 1.149994 & 1.977649 & -0.067737 \\ 6 & -0.504398 & 0.589611 & 1.206602 \\ 6 & -0.997761 & 2.919467 & 0.598655 \\ 6 & 0.290378 & 3.046462 & -0.024434 \\ 6 & 0.753579 & 0.657442 & 0.417705 \\ 6 & -0.88957 & -0.621374 & 1.753676 \\ 1 & -1.666643 & 3.784776 & 0.629862 \\ 1 & 0.591759 & 4.012264 & -0.441164 \\ 1 & 2.142117 & 2.083352 & -0.51545 \\ 6 & -1.637098 & -1.357927 & -0.760472 \\ 1 & -1.875449 & -0.685725 & 2.22775 \\ 6 & 0.073455 & -1.749268 & 2.031007 \\ 6 & 1.34467 & -1.715566 & 1.159927 \\ 1 & -0.429834 & -2.727756 & 1.924484 \\ 1 & 0.361622 & -1.694779 & 3.102739 \\ 1 & 1.1369 & -2.169848 & 0.177647 \\ 1 & 2.134407 & -2.332533 & 1.621543 \\ 6 & 1.878515 & -0.276102 & 0.953343 \\ 1 & -1.507519 & -2.358675 & -0.335215 \\ 6 & -0.580037 & -0.825742 & -1.517174 \\ 1 & 0.195996 & -1.525889 & -1.846361 \\ 1 & 0.245098 & 0.006508 & -0.639955 \\ 1 & -0.792853 & -0.033176 & -2.245551 \\ 6 & -2.991418 & -0.818462 & -0.67594 \\ 6 & -4.023912 & -1.609456 & -0.106798 \\ 6 & -5.342084 & -1.151199 & -0.043423 \\ 6 & -5.673672 & 0.12017 & -0.542475 \\ 6 & -4.666988 & 0.921091 & -1.102635 \\ 6 & -3.345707 & 0.463088 & -1.169807 \\ 1 & -3.776113 & -2.603825 & 0.279969 \\ 1 & -6.116934 & -1.787998 & 0.39404 \\ 1 & -6.705105 & 0.480612 & -0.495177 \\ 1 & -4.911914 & 1.913848 & -1.49233 \\ 1 & -2.578786 & 1.106726 & -1.605765 \\ 1 & 2.126777 & 0.122389 & 1.956186 \\ 6 & 3.175277 & -0.262327 & 0.152568 \\ \end{array}$
$6 \quad 3.234618 \quad-0.671402-1.195724$

$\begin{array}{lllll}6 & 4.448173 & -0.679655 & -1.897254\end{array}$

$\begin{array}{lllll}6 & 5.634507 & -0.279705 & -1.264215\end{array}$

$\begin{array}{llll}6 & 5.593591 & 0.131142 & 0.075379\end{array}$

$\begin{array}{lllll}6 & 4.375973 & 0.139751 & 0.771052\end{array}$

$\begin{array}{lllll}1 & 2.321426 & -0.984283 & -1.707794\end{array}$

$\begin{array}{lllll}1 & 4.46595 & -1.000628 & -2.943106\end{array}$

$\begin{array}{lllll}1 & 6.581527 & -0.287088 & -1.811353\end{array}$

$\begin{array}{llll}1 & 6.510094 & 0.448079 & 0.581895\end{array}$

$\begin{array}{llll}1 & 4.354322 & 0.464602 & 1.816732\end{array}$

\section{0b)AHn + M: TS Ene1 \\ RBPW91/6-31G*}

$\begin{array}{llll}1 & 1.491747 & 1.513247 & -1.744346\end{array}$

$\begin{array}{lllll}6 & 0.799602 & 1.585112 & -0.900095\end{array}$

$\begin{array}{llll}6 & -1.060247 & 1.806519 & 1.226864\end{array}$

$\begin{array}{lllll}6 & 0.106176 & 0.387454 & -0.484403\end{array}$

$\begin{array}{lllll}6 & 0.555579 & 2.799184 & -0.30178\end{array}$

$\begin{array}{llll}6 & -0.407512 & 2.918353 & 0.76047\end{array}$

$\begin{array}{llll}6 & -0.727395 & 0.468117 & 0.742234\end{array}$

$\begin{array}{llll}6 & 0.350581 & -0.84634 & -1.057415\end{array}$

$\begin{array}{lllll}1 & 1.070213 & 3.695859 & -0.659977\end{array}$

$\begin{array}{llll}1 & -0.630762 & 3.905898 & 1.174726\end{array}$

$\begin{array}{llll}1 & -1.813207 & 1.894502 & 2.017154\end{array}$

$\begin{array}{llll}6 & 2.121949 & -1.242412 & 1.10551\end{array}$

$\begin{array}{llll}1 & 1.076958 & -0.90562 & -1.87556\end{array}$

$\begin{array}{llll}6 & -0.544217 & -2.03911 & -0.836337\end{array}$

$\begin{array}{llll}6 & -1.247966 & -2.025542 & 0.532255\end{array}$

$\begin{array}{lllll}1 & 0.023141 & -2.980019 & -0.952805\end{array}$

$\begin{array}{lllll}1 & -1.304704 & -2.057574 & -1.6456\end{array}$

$\begin{array}{llll}1 & -0.52954 & -2.347108 & 1.303898\end{array}$

$\begin{array}{llll}1 & -2.071831 & -2.758883 & 0.544001\end{array}$

$\begin{array}{llll}6 & -1.802197 & -0.626761 & 0.929597\end{array}$

$\begin{array}{lllll}1 & 1.900836 & -2.292059 & 0.884757\end{array}$

$\begin{array}{llll}6 & 1.398516 & -0.623663 & 2.136258\end{array}$

$\begin{array}{lllll}1 & 0.844792 & -1.27152 & 2.826633\end{array}$

$\begin{array}{llll}1 & 0.244593 & 0.03371 & 1.548154\end{array}$

$\begin{array}{llll}1 & -2.038765 & -0.672549 & 2.009592\end{array}$

$\begin{array}{llll}6 & -3.121401 & -0.322115 & 0.214058\end{array}$

$\begin{array}{llll}6 & -4.334029 & -0.690509 & 0.832488\end{array}$

$\begin{array}{llll}6 & -5.567345 & -0.476861 & 0.20193\end{array}$

$\begin{array}{lllll}6 & -5.612617 & 0.115399 & -1.068721\end{array}$

$\begin{array}{lllll}6 & -4.415702 & 0.491098 & -1.694762\end{array}$

$\begin{array}{lllll}6 & -3.183273 & 0.276228 & -1.059908\end{array}$

$\begin{array}{lllll}1 & -4.309399 & -1.149404 & 1.827316\end{array}$

$\begin{array}{llll}1 & -6.493375 & -0.767178 & 0.707375\end{array}$

$\begin{array}{llll}1 & -6.572877 & 0.28845 & -1.563131\end{array}$

$\begin{array}{lllll}1 & -4.438141 & 0.960768 & -2.682769\end{array}$

$\begin{array}{lllll}1 & -2.260648 & 0.591175 & -1.553802\end{array}$

$\begin{array}{llll}1 & 1.809045 & 0.276624 & 2.610557\end{array}$

$\begin{array}{llll}6 & 3.278969 & -0.692372 & 0.406977\end{array}$

$\begin{array}{lllll}6 & 4.040379 & -1.533607 & -0.446935\end{array}$

$\begin{array}{lllll}6 & 5.176572 & -1.063618 & -1.10938\end{array}$

$\begin{array}{lllll}6 & 5.589996 & 0.269672 & -0.9454\end{array}$

$\begin{array}{lllll}6 & 4.849705 & 1.119473 & -0.10926\end{array}$

$\begin{array}{llll}6 & 3.712261 & 0.650198 & 0.55828\end{array}$

$1 \quad 3.726991 \quad-2.574668-0.579362$

$\begin{array}{lllll}1 & 5.745185 & -1.738678 & -1.756104\end{array}$

$\begin{array}{lllll}1 & 6.479368 & 0.640085 & -1.462878\end{array}$

$\begin{array}{llll}1 & 5.16102 & 2.159788 & 0.025836\end{array}$

$\begin{array}{llll}1 & 3.151054 & 1.331762 & 1.201202\end{array}$ 33b)소

UBPW $91 / 6-31 G^{*}$

$\begin{array}{llll}6 & -3.417468 & -0.680010 & 0.176262\end{array}$

$\begin{array}{llll}6 & -2.360185 & 0.276451 & 0.037755\end{array}$

$\begin{array}{lllll}6 & 0.096757 & 0.799877 & -0.461282\end{array}$

$\begin{array}{llll}6 & -1.538091 & 2.693002 & 0.059099\end{array}$

$\begin{array}{llll}6 & -0.156673 & 2.088982 & 0.364661\end{array}$

$\begin{array}{llll}6 & -2.619543 & 1.661623 & 0.160622\end{array}$

$\begin{array}{lllll}6 & -1.032020 & -0.212286 & -0.248472\end{array}$

$\begin{array}{lllll}6 & 1.496192 & 0.258044 & -0.189999\end{array}$

$\begin{array}{llll}1 & -1.746123 & 3.537848 & 0.741654\end{array}$

$\begin{array}{llll}1 & 0.642519 & 2.819506 & 0.156192\end{array}$

$\begin{array}{llll}6 & -3.186331 & -2.043355 & 0.031276\end{array}$

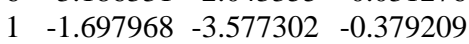

$\begin{array}{lllll}1 & 0.065833 & 1.098076 & -1.530078\end{array}$

$\begin{array}{lllll}1 & -1.528306 & 3.139722 & -0.959955\end{array}$

$\begin{array}{llll}1 & -0.096280 & 1.843288 & 1.439582\end{array}$

$\begin{array}{llll}1 & -3.651323 & 1.989979 & 0.323510\end{array}$

$\begin{array}{llll}6 & -0.831739 & -1.588310 & -0.395923\end{array}$

$\begin{array}{lllll}1 & -4.012116 & -2.752660 & 0.140369\end{array}$

$\begin{array}{llll}1 & -4.424462 & -0.312372 & 0.398928\end{array}$

$\begin{array}{llll}6 & -1.888490 & -2.507280 & -0.259334\end{array}$

$1 \quad 0.172659-1.957111-0.621094$

$\begin{array}{llll}6 & 4.131274 & -0.638869 & 0.335600\end{array}$

$\begin{array}{llll}6 & 1.844608 & -0.262889 & 1.073801\end{array}$

$\begin{array}{lllll}6 & 2.491668 & 0.314902 & -1.183048\end{array}$

$\begin{array}{lllll}6 & 3.798227 & -0.128062 & -0.926253\end{array}$

$\begin{array}{llll}6 & 3.147617 & -0.705921 & 1.335041\end{array}$

$\begin{array}{lllll}1 & 1.082405 & -0.331493 & 1.855903\end{array}$

$\begin{array}{lllll}1 & 2.239576 & 0.713839 & -2.171509\end{array}$

$14.554494 \quad-0.074370 \quad-1.715031$

$\begin{array}{llll}1 & 3.395822 & -1.106883 & 2.322331\end{array}$

$\begin{array}{llll}1 & 5.148447 & -0.985457 & 0.539579\end{array}$

34b)phenylethyl rad (HM•)

UBPW91/6-31G*

$\begin{array}{llll}6 & 0.465220 & -0.295452 & -0.000001\end{array}$

$\begin{array}{llll}6 & -2.313226 & 0.349185 & 0.000000\end{array}$

$\begin{array}{llll}6 & 0.014479 & 1.063743 & 0.000000\end{array}$

$\begin{array}{llll}6 & -0.541293 & -1.315028 & 0.000000\end{array}$

$\begin{array}{llll}6 & -1.896743 & -0.996845 & 0.000000\end{array}$

$\begin{array}{llll}6 & -1.345407 & 1.371406 & 0.000000\end{array}$

$\begin{array}{llll}1 & 0.750284 & 1.872247 & 0.000002\end{array}$

$\begin{array}{llll}1 & -0.225086 & -2.363325 & 0.000000\end{array}$

$\begin{array}{lllll}1 & -2.641575 & -1.798397 & 0.000001\end{array}$

$\begin{array}{lllll}1 & -1.661375 & 2.419154 & 0.000001\end{array}$

$\begin{array}{llll}1 & -3.378025 & 0.597396 & 0.000001\end{array}$

$\begin{array}{llll}6 & 1.841587 & -0.646528 & -0.000002\end{array}$

$\begin{array}{llll}1 & 2.085753 & -1.713759 & 0.000011\end{array}$

$\begin{array}{llll}6 & 2.977147 & 0.332352 & -0.000001\end{array}$

$\begin{array}{lllll}1 & 2.955533 & 0.997263 & -0.885357\end{array}$

$\begin{array}{llll}1 & 2.955630 & 0.997148 & 0.885447\end{array}$

$\begin{array}{lllll}1 & 3.948282 & -0.184721 & -0.000085\end{array}$

35b)•M2•+M:TS

UBPW91/6-31G*

$\begin{array}{llll}1 & -2.817720 & 5.442068 & 0.451817\end{array}$

$\begin{array}{llll}6 & -2.474264 & 4.480939 & 0.057468\end{array}$ 
$\begin{array}{rrrr}6 & -1.586465 & 2.014016 & -0.943999 \\ 6 & -1.502034 & 3.757888 & 0.749931 \\ 6 & -3.012405 & 3.978437 & -1.139995 \\ 6 & -2.560217 & 2.739931 & -1.630604 \\ 6 & -1.024068 & 2.502947 & 0.271231 \\ 1 & -1.088300 & 4.156947 & 1.682020 \\ 1 & -3.775441 & 4.542484 & -1.683301 \\ 1 & -2.975720 & 2.336864 & -2.559067 \\ 6 & -0.005539 & 1.807408 & 1.017101 \\ 1 & -1.260479 & 1.050334 & -1.343362 \\ 6 & 0.558838 & 0.537548 & 0.739369 \\ 1 & 0.309696 & 2.293012 & 1.950688 \\ 1 & -1.329037 & -2.997477 & -0.398607 \\ 6 & -0.832758 & -1.528322 & 1.925980 \\ 6 & 1.939903 & 0.201278 & 1.339236 \\ 1 & 0.443392 & 0.173605 & -0.294292 \\ 1 & 2.029189 & -0.887864 & 1.489798 \\ 1 & 1.986401 & 0.655656 & 2.351024 \\ 1 & -2.808844 & -0.886887 & 2.460066 \\ 1 & -6.253996 & -2.172831 & -0.444077 \\ 1 & -5.187350 & -3.529290 & -2.261536 \\ 1 & -4.866283 & -1.229799 & 1.385512 \\ 1 & -0.236897 & -0.413573 & 1.333744 \\ 6 & -4.578556 & -3.113839 & -1.453865 \\ 6 & -5.175150 & -2.353859 & -0.433191 \\ 1 & -2.716322 & -3.924955 & -2.217664 \\ 6 & -3.189581 & -3.334966 & -1.426974 \\ 6 & -2.406101 & -2.809613 & -0.398435 \\ 6 & -4.395161 & -1.823950 & 0.595897 \\ 1 & -0.468753 & -1.333079 & 2.943914 \\ 6 & -2.230433 & -1.480643 & 1.742424 \\ 6 & -2.988001 & -2.036871 & 0.647342 \\ 6 & 3.048415 & 0.714897 & 0.474612 \\ 6 & 4.197357 & 0.006747 & 0.030465 \\ 6 & 5.142371 & 0.666997 & -0.823683 \\ 6 & 6.286375 & 0.020997 & -1.282996 \\ 6 & 6.546700 & -1.314518 & -0.918077 \\ 6 & 5.637424 & -1.988116 & -0.080563 \\ 6 & 4.490264 & -1.349836 & 0.387506 \\ 1 & 4.946734 & 1.704179 & -1.114226 \\ 1 & 6.985619 & 0.555747 & -1.932849 \\ 1 & 7.445000 & -1.821888 & -1.279692 \\ 1 & 5.832310 & -3.024844 & 0.210580 \\ 1 & 3.806659 & -1.896766 & 1.041850 \\ 1 & 2.943401 & 1.752264 & 0.136722 \\ 1 & -0.264739 & -2.316898 & 1.413868\end{array}$

36b)M2•

UBPW91/6-31G*

$\begin{array}{llll}1 & -6.345023 & -0.853658 & 0.575209\end{array}$

$\begin{array}{llll}6 & -5.431609 & -0.297786 & 0.344221\end{array}$

$\begin{array}{llll}6 & -3.079188 & 1.108242 & -0.240876\end{array}$

$\begin{array}{llll}6 & -4.208567 & -0.974286 & 0.263841\end{array}$

$\begin{array}{llll}6 & -5.484288 & 1.086917 & 0.130232\end{array}$

$\begin{array}{lllll}6 & -4.299949 & 1.784627 & -0.161222\end{array}$

$\begin{array}{llll}6 & -3.006593 & -0.290583 & -0.034686\end{array}$

$\begin{array}{llll}1 & -4.175487 & -2.055964 & 0.430811\end{array}$

$\begin{array}{llll}1 & -6.436825 & 1.620321 & 0.193625\end{array}$

$\begin{array}{lllll}1 & -4.328631 & 2.866295 & -0.322767\end{array}$

$\begin{array}{lllll}6 & -1.755173 & -1.058699 & -0.112725\end{array}$

$\begin{array}{lllll}1 & -2.168377 & 1.673448 & -0.456181\end{array}$ $\begin{array}{llll}6 & -0.537762 & -0.622340 & -0.510247\end{array}$

$\begin{array}{llll}1 & -1.846663 & -2.114925 & 0.175940\end{array}$

$\begin{array}{lllll}6 & 0.711681 & -1.479117 & -0.525031\end{array}$

$\begin{array}{lllll}1 & -0.392259 & 0.420755 & -0.814415\end{array}$

$\begin{array}{lllll}1 & 1.154420 & -1.483239 & -1.536542\end{array}$

$\begin{array}{lllll}1 & 0.434346 & -2.526283 & -0.297604\end{array}$

$\begin{array}{llll}6 & 1.698679 & -0.981623 & 0.496713\end{array}$

$\begin{array}{llll}6 & 2.941238 & -0.337983 & 0.256352\end{array}$

$\begin{array}{llll}6 & 3.734665 & 0.097932 & 1.369693\end{array}$

$\begin{array}{llll}6 & 4.965322 & 0.721483 & 1.187676\end{array}$

$\begin{array}{lllll}6 & 5.466730 & 0.945167 & -0.109828\end{array}$

$\begin{array}{lllll}6 & 4.708634 & 0.531262 & -1.221600\end{array}$

$6 \quad 3.475246-0.094831 \quad-1.051123$

$\begin{array}{llll}1 & 3.352153 & -0.071884 & 2.381193\end{array}$

$\begin{array}{llll}1 & 5.544782 & 1.039964 & 2.059362\end{array}$

$1 \quad 6.433218 \quad 1.435769-0.252055$

$\begin{array}{lllll}1 & 5.089080 & 0.702770 & -2.233079\end{array}$

$\begin{array}{lllll}1 & 2.907497 & -0.401698 & -1.933206\end{array}$

$\begin{array}{llll}1 & 1.383379 & -1.081959 & 1.541352\end{array}$

\section{7b) $\underline{H M \bullet+M: T S}$}

UBPW91/6-31G*

$1-3.860655-2.034351-0.538275$

$\begin{array}{lllll}6 & -3.801626 & -0.965105 & -0.309251\end{array}$

$\begin{array}{llll}6 & -3.656056 & 1.764146 & 0.276093\end{array}$

$\begin{array}{llll}6 & -4.936247 & -0.291434 & 0.152232\end{array}$

$\begin{array}{llll}6 & -2.564400 & -0.293665 & -0.492948\end{array}$

$\begin{array}{lllll}6 & -2.522017 & 1.092798 & -0.187224\end{array}$

$\begin{array}{llll}6 & -4.871315 & 1.079181 & 0.449632\end{array}$

$\begin{array}{lllll}1 & -5.875565 & -0.837117 & 0.282127\end{array}$

$\begin{array}{lllll}6 & -1.414057 & -1.042476 & -0.982105\end{array}$

$\begin{array}{lllll}1 & -1.589313 & 1.647976 & -0.318238\end{array}$

$\begin{array}{llll}1 & -5.756463 & 1.609057 & 0.812251\end{array}$

$\begin{array}{llll}1 & -3.594754 & 2.832625 & 0.503962\end{array}$

$\begin{array}{llll}6 & -0.137481 & -0.569296 & -1.192412\end{array}$

$\begin{array}{llll}6 & -1.593700 & -2.112350 & -1.145070\end{array}$

$1 \quad 0.596015 \quad-1.201460 \quad-1.697327$

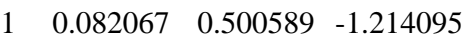

$\begin{array}{llll}1 & 1.423294 & -2.439015 & 2.101865\end{array}$

$\begin{array}{llll}1 & 1.380102 & -2.864792 & 0.378400\end{array}$

$\begin{array}{llll}1 & -0.128586 & -2.455201 & 1.227160\end{array}$

$\begin{array}{lllll}6 & 1.102479 & -0.740902 & 0.798209\end{array}$

$\begin{array}{llll}6 & 2.364714 & -0.153004 & 0.418877\end{array}$

$\begin{array}{lllll}1 & 5.752049 & 1.557509 & -0.656073\end{array}$

$\begin{array}{llll}6 & 0.937214 & -2.196551 & 1.135977\end{array}$

$\begin{array}{llll}6 & 4.812127 & 1.084358 & -0.359186\end{array}$

$\begin{array}{llll}6 & 2.525554 & 1.264419 & 0.444119\end{array}$

$\begin{array}{lllll}6 & 3.481716 & -0.925582 & -0.013732\end{array}$

$\begin{array}{lllll}6 & 4.679260 & -0.314748 & -0.393077\end{array}$

$\begin{array}{llll}6 & 3.723556 & 1.869018 & 0.063979\end{array}$

$\begin{array}{llll}1 & 1.686675 & 1.882992 & 0.780552\end{array}$

$\begin{array}{lllll}1 & 3.404982 & -2.015625 & -0.043599\end{array}$

$1 \quad 5.521287-0.934344 \quad-0.716434$

$\begin{array}{llll}1 & 3.816595 & 2.958606 & 0.101190\end{array}$

$\begin{array}{lllll}1 & 0.395447 & -0.062033 & 1.286509\end{array}$

38b) $M 2 \bullet+M: T S$

UBPW91/6-31G*

$\begin{array}{llll}1 & -0.021929 & 3.966882 & 1.648111\end{array}$

$\begin{array}{lllll}6 & -0.828918 & 3.972626 & 0.907981\end{array}$ $\begin{array}{llll}6 & -2.886929 & 3.990586 & -0.984208\end{array}$

$\begin{array}{llll}6 & -1.060102 & 5.119213 & 0.142737\end{array}$

$\begin{array}{llll}6 & -1.621541 & 2.804694 & 0.751299\end{array}$

$\begin{array}{lllll}6 & -2.659139 & 2.844658 & -0.218659\end{array}$

$\begin{array}{lllll}6 & -2.091003 & 5.136397 & -0.810456\end{array}$

$\begin{array}{lllll}1 & -0.432913 & 6.003764 & 0.288311\end{array}$

$\begin{array}{llll}6 & -1.343938 & 1.640604 & 1.580308\end{array}$

$\begin{array}{lllll}1 & -3.293685 & 1.967065 & -0.368619\end{array}$

$\begin{array}{lllll}1 & -2.273571 & 6.031407 & -1.411601\end{array}$

$\begin{array}{llll}1 & -3.693373 & 3.993698 & -1.723796\end{array}$

$\begin{array}{llll}6 & -1.992036 & 0.422434 & 1.552650\end{array}$

$\begin{array}{llll}1 & -0.486941 & 1.746974 & 2.256746\end{array}$

$\begin{array}{lllll}1 & -1.790204 & -0.309949 & 2.337407\end{array}$

$\begin{array}{llll}1 & -2.951352 & 0.299661 & 1.044885\end{array}$

$\begin{array}{llll}1 & 0.374935 & -1.745824 & 1.434085\end{array}$

$\begin{array}{lllll}1 & 0.813413 & -0.179920 & 0.731237\end{array}$

$\begin{array}{lllll}6 & -1.023957 & -0.969081 & -0.024736\end{array}$

$\begin{array}{lllll}6 & -1.987407 & -2.046831 & -0.130997\end{array}$

$\begin{array}{llll}1 & -4.718648 & -4.875565 & -0.432521\end{array}$

$\begin{array}{lllll}6 & 0.361407 & -1.157657 & 0.501295\end{array}$

$\begin{array}{llll}6 & -3.962637 & -4.090110 & -0.349057\end{array}$

$\begin{array}{lllll}6 & -3.095257 & -1.921394 & -1.018801\end{array}$

$\begin{array}{lllll}6 & -1.909980 & -3.238271 & 0.645587\end{array}$

$\begin{array}{llll}6 & -2.879397 & -4.238465 & 0.533990\end{array}$

$6-4.062818 \quad-2.921316 \quad-1.124832$

$\begin{array}{lllll}1 & -3.175907 & -1.019635 & -1.635028\end{array}$

$\begin{array}{llll}1 & -1.079559 & -3.375119 & 1.343522\end{array}$

$\begin{array}{llll}1 & -2.791371 & -5.144316 & 1.141458\end{array}$

$\begin{array}{llll}1 & -4.898217 & -2.796015 & -1.820199\end{array}$

$\begin{array}{llll}1 & -1.119637 & -0.195310 & -0.796037\end{array}$

$\begin{array}{lllll}6 & 1.309525 & -1.890472 & -0.552683\end{array}$

$\begin{array}{lllll}1 & 0.862519 & -2.874374 & -0.771893\end{array}$

$\begin{array}{lllll}1 & 1.284738 & -1.307488 & -1.488564\end{array}$

$\begin{array}{lllll}6 & 2.693630 & -2.050297 & -0.036788\end{array}$

$\begin{array}{lllll}1 & 2.890408 & -2.928817 & 0.589409\end{array}$

$\begin{array}{lllll}6 & 3.749948 & -1.104587 & -0.153543\end{array}$

$\begin{array}{lllll}6 & 3.623997 & 0.132140 & -0.865318\end{array}$

$\begin{array}{lllll}6 & 4.692066 & 1.023388 & -0.957076\end{array}$

$\begin{array}{lllll}6 & 5.928671 & 0.729144 & -0.352483\end{array}$

$\begin{array}{llll}6 & 6.080514 & -0.480239 & 0.353748\end{array}$

$\begin{array}{llll}6 & 5.018649 & -1.374804 & 0.455604\end{array}$

$\begin{array}{lllll}1 & 2.673365 & 0.384950 & -1.341955\end{array}$

$\begin{array}{lllll}1 & 4.564182 & 1.961482 & -1.505703\end{array}$

$\begin{array}{lllll}1 & 6.762280 & 1.432267 & -0.429711\end{array}$

$\begin{array}{llll}1 & 7.037079 & -0.718558 & 0.828556\end{array}$

$\begin{array}{llll}1 & 5.144297 & -2.311425 & 1.008518\end{array}$

41b)्AHn + HM・: TS tr1a UBPW91/6-31G*

$\begin{array}{llll}6 & 1.079856 & 3.305923 & -0.273559\end{array}$

$\begin{array}{llll}6 & 1.033652 & 2.097428 & 0.534343\end{array}$

$\begin{array}{llll}6 & 0.689423 & -0.457314 & 0.590346\end{array}$

$\begin{array}{llll}6 & 1.755841 & 0.787221 & 2.582396\end{array}$

$\begin{array}{lllll}6 & 0.768507 & -0.304151 & 2.134578\end{array}$

$\begin{array}{llll}6 & 1.633383 & 2.044189 & 1.767455\end{array}$

$\begin{array}{lllll}6 & 0.31082 & 0.906116 & -0.042823\end{array}$

$\begin{array}{llll}1 & 1.606827 & 1.007587 & 3.656232\end{array}$

$1 \quad 1.036856-1.272545 \quad 2.589426$

$\begin{array}{lllll}6 & 0.741939 & 3.294079 & -1.60059\end{array}$

$\begin{array}{llll}1 & -0.859547 & 1.029161 & 0.3327\end{array}$

$\begin{array}{llll}1 & 0.159454 & 2.062242 & -3.326896\end{array}$ 
$\begin{array}{llll}1 & 2.797298 & 0.410396 & 2.507134 \\ 1 & -0.239617 & -0.045493 & 2.502783 \\ 1 & 2.119585 & 2.949028 & 2.152365 \\ 6 & 0.175136 & 0.918553 & -1.51921 \\ 1 & 0.829468 & 4.206957 & -2.197251 \\ 1 & 1.472761 & 4.217598 & 0.189751 \\ 6 & 0.32781 & 2.065969 & -2.245774 \\ 1 & -0.13252 & -0.012558 & -2.005336 \\ 1 & -2.244677 & 1.18824 & 1.863687 \\ 6 & -2.448991 & 1.14173 & 0.78571 \\ 6 & -2.743981 & 2.466408 & 0.125875 \\ 1 & -2.526157 & 2.450619 & -0.954819 \\ 1 & -3.809869 & 2.746236 & 0.241381 \\ 1 & -2.137378 & 3.269637 & 0.570299 \\ 6 & -3.025032 & -0.102893 & 0.323791 \\ 6 & -3.05119 & -1.245957 & 1.177882 \\ 6 & -3.574604 & -2.465727 & 0.74853 \\ 6 & -4.094423 & -2.598158 & -0.552441 \\ 6 & -4.082211 & -1.486794 & -1.412379 \\ 6 & -3.559634 & -0.262055 & -0.98802 \\ 1 & -2.660428 & -1.15061 & 2.196367 \\ 1 & -3.586828 & -3.32022 & 1.432022 \\ 1 & -4.506874 & -3.553336 & -0.888604 \\ 1 & -4.488522 & -1.57669 & -2.424533 \\ 1 & -3.567681 & 0.589556 & -1.672662 \\ 1 & -0.148259 & -1.148175 & 0.383121 \\ 6 & 1.946031 & -1.116986 & 0.019758 \\ 6 & 2.01468 & -2.526084 & -0.000869 \\ 6 & 3.151892 & -3.194083 & -0.473725 \\ 6 & 4.252286 & -2.461072 & -0.942722 \\ 6 & 4.197763 & -1.0605 & -0.934338 \\ 6 & 3.057324 & -0.394971 & -0.458736 \\ 1 & 1.156653 & -3.107317 & 0.354703 \\ 1 & 3.174664 & -4.288043 & -0.483473 \\ 1 & 5.14082 & -2.977531 & -1.317387 \\ 1 & 5.046382 & -0.476858 & -1.303971 \\ 1 & 3.029938 & 0.696883 & -0.469899 \\ & & & \end{array}$

42b) $\underline{A H x}+H M \bullet:$ TS tr1a UBPW91/6-31G*

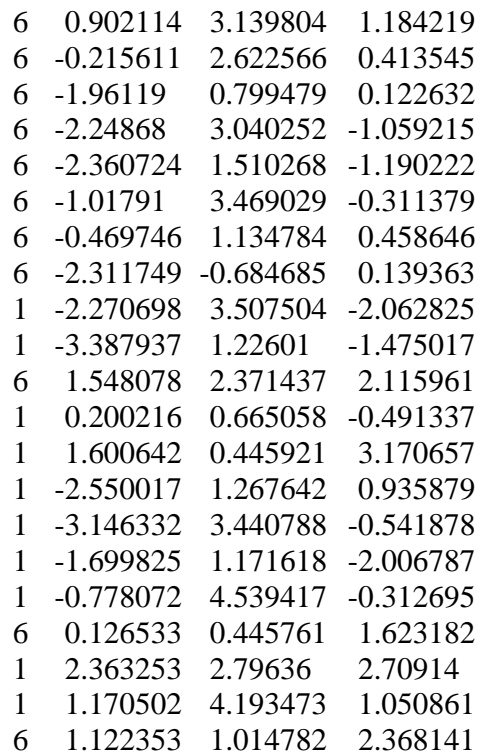

$\begin{array}{llll}1 & -0.180935 & -0.585469 & 1.814222\end{array}$

$\begin{array}{llll}6 & -3.023179 & -3.433553 & 0.240968\end{array}$

$\begin{array}{lllll}6 & -2.230456 & -1.500983 & -1.007161\end{array}$

$\begin{array}{llll}6 & -2.772 & -1.278148 & 1.333858\end{array}$

$\begin{array}{lllll}6 & -3.119605 & -2.63488 & 1.389684\end{array}$

$\begin{array}{llll}6 & -2.580956 & -2.858882 & -0.95906\end{array}$

$\begin{array}{lllll}1 & -1.90801 & -1.071701 & -1.959069\end{array}$

$\begin{array}{llll}1 & -2.862832 & -0.660472 & 2.233567\end{array}$

$\begin{array}{llll}1 & -3.474683 & -3.065555 & 2.330651\end{array}$

$\begin{array}{llll}1 & -2.514617 & -3.466524 & -1.866593\end{array}$

$\begin{array}{llll}1 & -3.299189 & -4.491215 & 0.278148\end{array}$

$\begin{array}{llll}1 & 0.331724 & -0.444137 & -2.189278\end{array}$

$\begin{array}{llll}6 & 1.143725 & 0.107555 & -1.698867\end{array}$

$\begin{array}{llll}6 & 1.558712 & 1.388797 & -2.381322\end{array}$

$\begin{array}{lllll}1 & 2.041187 & 2.095059 & -1.685483\end{array}$

$\begin{array}{llll}1 & 2.276787 & 1.197372 & -3.203618\end{array}$

$\begin{array}{lllll}1 & 0.690312 & 1.904967 & -2.818617\end{array}$

$\begin{array}{lllll}6 & 2.094264 & -0.733013 & -0.995379\end{array}$

$\begin{array}{lllll}6 & 1.732373 & -2.059063 & -0.619877\end{array}$

$\begin{array}{lllll}6 & 2.621605 & -2.89002 & 0.063881\end{array}$

$\begin{array}{llll}6 & 3.906766 & -2.430856 & 0.402035\end{array}$

$\begin{array}{llll}6 & 4.28393 & -1.12377 & 0.048104\end{array}$

$\begin{array}{lllll}6 & 3.396535 & -0.286924 & -0.632968\end{array}$

$\begin{array}{lllll}1 & 0.734301 & -2.426462 & -0.877497\end{array}$

$\begin{array}{llll}1 & 2.314441 & -3.905226 & 0.333293\end{array}$

$\begin{array}{llll}1 & 4.605105 & -3.082802 & 0.934287\end{array}$

$\begin{array}{llll}1 & 5.281956 & -0.755531 & 0.305272\end{array}$

$\begin{array}{llll}1 & 3.711634 & 0.72549 & -0.897903\end{array}$

43a) cis A-Sty

RBPW91/6-31G*

$\begin{array}{llll}6-0.039453 & 3.171871 & 0.054125\end{array}$

$6-0.143548 \quad 1.816866-0.330398$

$\begin{array}{llll}6 & -1.661878 & -0.145204 & -1.070522\end{array}$

$\begin{array}{lllll}6 & 0.820722 & -0.092249 & -1.649413\end{array}$

$\begin{array}{llll}6 & -0.355475 & -0.966346 & -1.197327\end{array}$

$\begin{array}{llll}6 & 1.125204 & 1.014215 & -0.614480\end{array}$

$\begin{array}{llll}6 & -1.436092 & 1.253147 & -0.478416\end{array}$

$\begin{array}{llll}6 & -2.740652 & -0.959054 & -0.359399\end{array}$

$\begin{array}{llll}1 & 1.715959 & -0.712513 & -1.816624\end{array}$

$\begin{array}{llll}1 & -0.523625 & -1.794919 & -1.906280\end{array}$

$\begin{array}{llll}6 & -1.166802 & 3.950917 & 0.333068\end{array}$

$\begin{array}{llll}1-3.337385 & 3.971280 & 0.433930\end{array}$

$1-2.024755 \quad 0.027900 \quad-2.103844$

$\begin{array}{lllll}1 & 0.574640 & 0.383125 & -2.617036\end{array}$

$\begin{array}{llll}1 & -0.124879 & -1.440722 & -0.227977\end{array}$

$\begin{array}{llll}6 & -2.565480 & 2.049176 & -0.192216\end{array}$

$\begin{array}{llll}1-1.050436 & 4.997597 & 0.629057\end{array}$

$\begin{array}{llll}1 & 0.955208 & 3.623043 & 0.126857\end{array}$

$\begin{array}{llll}6 & -2.442619 & 3.379624 & 0.219330\end{array}$

$\begin{array}{llll}1 & -3.560656 & 1.608586 & -0.305235\end{array}$

$\begin{array}{llll}6 & -4.678219 & -2.575397 & 0.927330\end{array}$

$6-2.723913-1.131934 \quad 1.039866$

$6-3.747786-1.607941-1.098102$

$6-4.708954-2.409976-0.464136$

$6-3.681525-1.931350 \quad 1.677439$

$\begin{array}{llll}1 & -1.959129 & -0.622021 & 1.633856\end{array}$

$1-3.778855-1.483131-2.186004$

$1-5.483799-2.902001-1.059626$

$\begin{array}{llll}1 & -3.651977 & -2.049460 & 2.764829\end{array}$

$\begin{array}{llll}1 & -5.427214 & -3.197581 & 1.425593\end{array}$ $\begin{array}{llll}1 & 1.863222 & 1.709707 & -1.057014\end{array}$

$\begin{array}{llll}6 & 1.792752 & 0.428741 & 0.689084\end{array}$

$\begin{array}{llll}1 & 1.065589 & -0.266923 & 1.145295\end{array}$

$\begin{array}{llll}6 & 2.114659 & 1.498915 & 1.754583\end{array}$

$\begin{array}{llll}1 & 2.585008 & 1.027750 & 2.632735\end{array}$

$\begin{array}{llll}1 & 2.819976 & 2.254826 & 1.369288\end{array}$

$\begin{array}{llll}1 & 1.205756 & 2.020752 & 2.089820\end{array}$

$\begin{array}{llll}6 & 3.050315 & -0.377235 & 0.366809\end{array}$

$\begin{array}{llll}6 & 3.138218 & -1.740342 & 0.710924\end{array}$

$\begin{array}{lllll}6 & 4.298432 & -2.484456 & 0.449017\end{array}$

$\begin{array}{lllll}6 & 5.400153 & -1.875491 & -0.167352\end{array}$

$\begin{array}{lllll}6 & 5.330122 & -0.518068 & -0.516367\end{array}$

$\begin{array}{lllll}6 & 4.169462 & 0.220727 & -0.250060\end{array}$

$\begin{array}{llll}1 & 2.284367 & -2.225402 & 1.195801\end{array}$

$\begin{array}{lllll}1 & 4.339190 & -3.541690 & 0.727804\end{array}$

$16.306375 \quad-2.452038-0.373896$

$\begin{array}{lllll}1 & 6.184240 & -0.030876 & -0.996383\end{array}$

$\begin{array}{lllll}1 & 4.137321 & 1.279770 & -0.525264\end{array}$

44b)trans A-Sty

RBPW91/6-31G*

$\begin{array}{llll}6 & 0.20631 & 2.84892 & 0.70674\end{array}$

$\begin{array}{llll}6 & 0.255629 & 1.534809 & 0.188458\end{array}$

$\begin{array}{lllll}6 & 1.62573 & -0.281791 & -1.045231\end{array}$

$\begin{array}{llll}6 & -0.386835 & -0.891574 & 0.403297\end{array}$

$\begin{array}{lllll}6 & 0.353537 & -1.163619 & -0.911934\end{array}$

$\begin{array}{llll}6 & -0.893079 & 0.566551 & 0.468983\end{array}$

$\begin{array}{llll}6 & 1.392158 & 1.152265 & -0.566567\end{array}$

$1-1.230618 \quad-1.590192 \quad 0.51829$

$\begin{array}{lllll}1 & 0.638346 & -2.226329 & -0.982835\end{array}$

$\begin{array}{llll}6 & 1.210209 & 3.7861 & 0.444499\end{array}$

$\begin{array}{llll}1 & 3.099309 & 4.139372 & -0.57148\end{array}$

$\begin{array}{llll}1 & 0.296705 & -1.077673 & 1.250814\end{array}$

$\begin{array}{llll}1 & -0.314755 & -0.961922 & -1.767559\end{array}$

$\begin{array}{lllll}6 & 2.394342 & 2.109589 & -0.831811\end{array}$

$\begin{array}{llll}1 & 1.139162 & 4.795538 & 0.860034\end{array}$

$\begin{array}{llll}1 & -0.641721 & 3.134745 & 1.336783\end{array}$

$\begin{array}{llll}6 & 2.307788 & 3.417998 & -0.348731\end{array}$

$\begin{array}{lllll}1 & 3.269916 & 1.804445 & -1.414358\end{array}$

$\begin{array}{llll}1-1.248209 & 0.76594 & 1.497861\end{array}$

$\begin{array}{llll}6 & -2.135705 & 0.785993 & -0.48007\end{array}$

$\begin{array}{llll}1 & -1.80099 & 0.568411 & -1.510447\end{array}$

$\begin{array}{llll}6 & -2.677331 & 2.231999 & -0.486606\end{array}$

$\begin{array}{llll}1 & -3.545421 & 2.304487 & -1.161659\end{array}$

$\begin{array}{llll}1-3.014307 & 2.545368 & 0.516237\end{array}$

$\begin{array}{llll}1 & -1.915056 & 2.947165 & -0.830166\end{array}$

$6-3.267962-0.187628-0.154823$

$\begin{array}{llll}6 & -3.738217 & -1.094621 & -1.12456\end{array}$

$6-4.797982-1.971558-0.848668$

$\begin{array}{llll}6 & -5.411274 & -1.9589 & 0.411631\end{array}$

$\begin{array}{llll}6 & -4.956115 & -1.061057 & 1.389291\end{array}$

$6-3.898859-0.185706 \quad 1.106908$

$1-3.267193-1.11159-2.113183$

$1-5.143135-2.6653 \quad-1.621163$

$1-6.237253-2.64142 \quad 0.631128$

$\begin{array}{llll}1-5.428025 & -1.040088 & 2.376261\end{array}$

$\begin{array}{llll}1-3.562553 & 0.511118 & 1.881267\end{array}$

$\begin{array}{llll}1 & 1.88792 & -0.238459 & -2.119122\end{array}$

$\begin{array}{lllll}6 & 2.817624 & -0.943541 & -0.33883\end{array}$

$\begin{array}{lllll}6 & 3.584769 & -1.900472 & -1.034355\end{array}$

$\begin{array}{lllll}6 & 4.642367 & -2.575258 & -0.410031\end{array}$ 
$\begin{array}{llll}6 & 4.955564 & -2.304313 & 0.930492\end{array}$

$\begin{array}{lllll}6 & 4.205638 & -1.350757 & 1.633098\end{array}$

$\begin{array}{llll}6 & 3.149882 & -0.674588 & 1.00322\end{array}$

$\begin{array}{lllll}1 & 3.34791 & -2.118057 & -2.081785\end{array}$

$\begin{array}{lllll}1 & 5.226435 & -3.309302 & -0.973343\end{array}$

$15.781974-2.827336 \quad 1.420341$

$\begin{array}{lllll}1 & 4.445042 & -1.125142 & 2.676668\end{array}$

$\begin{array}{llll}1 & 2.587378 & 0.083177 & 1.555024\end{array}$ 
Table 3. Raw data from Figures 12 and 13. B3LYP/6-31G(d) and BPW91/6-31G(d) constrained optimizations of two molecules of styrene in the gauche/sickle conformation. Bonds lengths $\mathrm{A} / \mathrm{B}$ or $\mathrm{A} / \mathrm{C}$ were constrained.

\begin{tabular}{|c|c|c|c|c|c|c|c|}
\hline \multicolumn{2}{|c|}{ Bond $(\AA)$} & \multicolumn{2}{|c|}{ Energy, 0K (au) } & \multicolumn{2}{|c|}{ Bond (Å) } & \multicolumn{2}{|c|}{ Energy, 0K (au) } \\
\hline A & B & B3LYP & BPW91 & A & $\mathbf{C}$ & B3LYP & BPW91 \\
\hline 1.5 & 1.6 & -619.296421 & -619.205166 & 1.5 & 1.6 & -619.312378 & -619.220269 \\
\hline 1.5 & 1.7 & -619.292639 & -619.201994 & 1.5 & 1.7 & -619.309664 & -619.218123 \\
\hline 1.5 & 1.8 & -619.285894 & -619.196039 & 1.5 & 1.8 & -619.303406 & -619.212597 \\
\hline 1.5 & 1.9 & -619.278104 & -619.189192 & 1.5 & 1.9 & -619.295525 & -619.205573 \\
\hline 1.5 & 2.0 & -619.270500 & -619.182629 & 1.5 & 2.0 & -619.287198 & -619.198193 \\
\hline 1.5 & 2.1 & -619.263788 & -619.176992 & 1.5 & 2.1 & -619.279098 & -619.191093 \\
\hline 1.5 & 2.2 & -619.258292 & -619.172541 & 1.5 & 2.2 & -619.271572 & -619.184592 \\
\hline 1.5 & 2.3 & -619.254057 & -619.169243 & 1.5 & 2.3 & -619.264762 & -619.178818 \\
\hline 1.5 & 2.4 & -619.251638 & -619.166921 & 1.5 & 2.4 & -619.259984 & -619.173785 \\
\hline 1.5 & 2.5 & -619.251837 & -619.165341 & 1.5 & 2.5 & -619.258215 & -619.169470 \\
\hline 1.5 & 2.6 & -619.253156 & -619.164336 & 1.5 & 2.6 & -619.258115 & -619.167030 \\
\hline 1.5 & 2.7 & -619.254817 & -619.164248 & 1.5 & 2.7 & -619.258789 & -619.166217 \\
\hline 1.5 & 2.8 & -619.256454 & -619.164658 & 1.5 & 2.8 & -619.259655 & -619.166172 \\
\hline 1.5 & 2.9 & -619.257877 & -619.165251 & 1.5 & 2.9 & -619.260420 & -619.166442 \\
\hline 1.5 & 3.0 & -619.259025 & -619.165870 & 1.5 & 3.0 & -619.260929 & -619.166758 \\
\hline 1.5 & 3.1 & -619.259891 & -619.166391 & 1.5 & 3.1 & -619.260997 & -619.166763 \\
\hline 1.5 & 3.2 & -619.260478 & -619.166748 & 1.5 & 3.2 & -619.260568 & -619.167202 \\
\hline 1.5 & 3.3 & -619.260862 & -619.166997 & 1.5 & 3.3 & -619.261112 & -619.166765 \\
\hline 1.5 & 3.4 & -619.261100 & -619.167172 & 1.5 & 3.4 & -619.259555 & -619.165955 \\
\hline 1.6 & 1.6 & -619.296690 & -619.205786 & 1.6 & 1.6 & -619.312444 & -619.220604 \\
\hline 1.6 & 1.7 & -619.293143 & -619.202908 & 1.6 & 1.7 & -619.309520 & -619.218248 \\
\hline 1.6 & 1.8 & -619.286792 & -619.197372 & 1.6 & 1.8 & -619.303070 & -619.212532 \\
\hline 1.6 & 1.9 & -619.279495 & -619.191027 & 1.6 & 1.9 & -619.295007 & -619.205329 \\
\hline 1.6 & 2.0 & -619.272464 & -619.185028 & 1.6 & 2.0 & -619.286507 & -619.197782 \\
\hline 1.6 & 2.1 & -619.266396 & -619.179991 & 1.6 & 2.1 & -619.278248 & -619.190534 \\
\hline 1.6 & 2.2 & -619.261597 & -619.176150 & 1.6 & 2.2 & -619.270575 & -619.183902 \\
\hline 1.6 & 2.3 & -619.258070 & -619.173452 & 1.6 & 2.3 & -619.263631 & -619.178012 \\
\hline 1.6 & 2.4 & -619.255808 & -619.171700 & 1.6 & 2.4 & -619.258906 & -619.172887 \\
\hline 1.6 & 2.5 & -619.255717 & -619.170633 & 1.6 & 2.5 & -619.257254 & -619.168557 \\
\hline 1.6 & 2.6 & -619.256795 & -619.170006 & 1.6 & 2.6 & -619.257346 & -619.166348 \\
\hline 1.6 & 2.7 & -619.258251 & -619.169653 & 1.6 & 2.7 & -619.258191 & -619.165798 \\
\hline 1.6 & 2.8 & -619.259707 & -619.169739 & 1.6 & 2.8 & -619.259229 & -619.166010 \\
\hline 1.6 & 2.9 & -619.260978 & -619.170055 & 1.6 & 2.9 & -619.259591 & -619.166519 \\
\hline 1.6 & 3.0 & -619.261998 & -619.170433 & 1.6 & 3.0 & -619.262149 & -619.168918 \\
\hline 1.6 & 3.1 & -619.262765 & -619.170763 & 1.6 & 3.1 & -619.263110 & -619.170000 \\
\hline 1.6 & 3.2 & -619.263269 & -619.170967 & 1.6 & 3.2 & -619.263608 & -619.170729 \\
\hline 1.6 & 3.3 & -619.263548 & -619.171034 & 1.6 & 3.3 & -619.263609 & -619.171035 \\
\hline 1.6 & 3.4 & -619.263658 & -619.170989 & 1.6 & 3.4 & -619.263088 & -619.170725 \\
\hline 1.7 & 1.6 & -619.290731 & -619.200321 & 1.8 & 1.6 & -619.294871 & -619.203961 \\
\hline 1.7 & 1.7 & -619.287406 & -619.197730 & 1.8 & 1.7 & -619.291558 & -619.201198 \\
\hline 1.7 & 1.8 & -619.281486 & -619.192661 & 1.8 & 1.8 & -619.284775 & -619.195161 \\
\hline 1.7 & 1.9 & -619.274789 & -619.186915 & 1.8 & 1.9 & -619.276416 & -619.187672 \\
\hline 1.7 & 2.0 & -619.268474 & -619.181605 & 1.8 & 2.0 & -619.267640 & -619.179871 \\
\hline
\end{tabular}




\begin{tabular}{|c|c|c|c|c|c|c|c|}
\hline 1.7 & 2.1 & -619.263219 & -619.177315 & 1.8 & 2.1 & -619.259131 & -619.172406 \\
\hline 1.7 & 2.2 & -619.259285 & -619.174221 & 1.8 & 2.2 & -619.251254 & -619.165624 \\
\hline 1.7 & 2.3 & -619.256636 & -619.172230 & 1.8 & 2.3 & -619.244169 & -619.159645 \\
\hline 1.7 & 2.4 & -619.255055 & -619.171130 & 1.8 & 2.4 & -619.240021 & -619.154492 \\
\hline 1.7 & 2.5 & -619.254649 & -619.170659 & 1.8 & 2.5 & -619.239299 & -619.150476 \\
\hline 1.7 & 2.6 & -619.255453 & -619.170552 & 1.8 & 2.6 & -619.240335 & -619.149129 \\
\hline 1.7 & 2.7 & -619.256689 & -619.170601 & 1.8 & 2.7 & -619.242217 & -619.149559 \\
\hline 1.7 & 2.8 & -619.257960 & -619.170688 & 1.8 & 2.8 & -619.245533 & -619.154492 \\
\hline 1.7 & 2.9 & -619.259079 & -619.170763 & 1.8 & 2.9 & -619.249385 & \\
\hline 1.7 & 3.0 & -619.259974 & -619.170885 & 1.8 & 3.0 & -619.252448 & \\
\hline 1.7 & 3.1 & -619.260638 & -619.171000 & 1.8 & 3.1 & -619.254835 & -619.167267 \\
\hline 1.7 & 3.2 & -619.261068 & -619.171035 & 1.8 & 3.2 & -619.256545 & -619.169482 \\
\hline 1.7 & 3.3 & -619.261276 & -619.170972 & 1.8 & 3.3 & -619.257562 & -619.170728 \\
\hline 1.7 & 3.4 & -619.261310 & -619.170813 & 1.8 & 3.4 & -619.257855 & -619.171061 \\
\hline 1.8 & 1.6 & -619.281279 & -619.191536 & 2.0 & 1.6 & -619.268918 & -619.179361 \\
\hline 1.8 & 1.7 & -619.278384 & -619.189406 & 2.0 & 1.7 & -619.265212 & -619.176226 \\
\hline 1.8 & 1.8 & -619.272992 & -619.184896 & 2.0 & 1.8 & -619.258093 & -619.169836 \\
\hline 1.8 & 1.9 & -619.267041 & -619.179876 & 2.0 & 1.9 & -619.249477 & -619.162089 \\
\hline 1.8 & 2.0 & -619.261637 & -619.175408 & 2.0 & 2.0 & -619.240515 & -619.154112 \\
\hline 1.8 & 2.1 & -619.257427 & -619.172038 & 2.0 & 2.1 & -619.231881 & -619.146539 \\
\hline 1.8 & 2.2 & -619.254619 & -619.169876 & 2.0 & 2.2 & -619.223948 & -619.139738 \\
\hline 1.8 & 2.3 & -619.253097 & -619.168772 & 2.0 & 2.3 & -619.217014 & -619.133867 \\
\hline 1.8 & 2.4 & -619.252565 & -619.168455 & 2.0 & 2.4 & -619.215476 & -619.129004 \\
\hline 1.8 & 2.5 & -619.252676 & -619.168653 & 2.0 & 2.5 & -619.218757 & -619.127553 \\
\hline 1.8 & 2.6 & -619.253193 & -619.169138 & 2.0 & 2.6 & -619.223943 & -619.135885 \\
\hline 1.8 & 2.7 & -619.254140 & -619.169701 & 2.0 & 2.7 & -619.232490 & -619.145004 \\
\hline 1.8 & 2.8 & -619.255180 & -619.170211 & 2.0 & 2.8 & -619.240209 & -619.152460 \\
\hline 1.8 & 2.9 & -619.256107 & -619.170615 & 2.0 & 2.9 & -619.246511 & -619.158567 \\
\hline 1.8 & 3.0 & -619.256845 & -619.170900 & 2.0 & 3.0 & -619.251537 & -619.163489 \\
\hline 1.8 & 3.1 & -619.257381 & -619.171050 & 2.0 & 3.1 & -619.255428 & -619.167337 \\
\hline 1.8 & 3.2 & -619.257714 & -619.171055 & 2.0 & 3.2 & -619.258200 & -619.170189 \\
\hline 1.8 & 3.3 & -619.257851 & -619.170932 & 2.0 & 3.3 & -619.259948 & -619.172090 \\
\hline 1.8 & 3.4 & -619.257822 & -619.170705 & 2.0 & 3.4 & -619.260792 & -619.173138 \\
\hline 2.0 & 1.6 & -619.258739 & -619.170832 & 2.2 & 1.6 & -619.242767 & -619.154792 \\
\hline 2.0 & 1.7 & -619.257235 & -619.170056 & 2.2 & 1.7 & -619.238774 & -619.151396 \\
\hline 2.0 & 1.8 & -619.253573 & -619.167197 & 2.2 & 1.8 & -619.231445 & -619.144826 \\
\hline 2.0 & 1.9 & -619.249723 & -619.164089 & 2.2 & 1.9 & -619.222691 & -619.136954 \\
\hline 2.0 & 2.0 & -619.246888 & -619.161808 & 2.2 & 2.0 & -619.213664 & -619.128917 \\
\hline 2.0 & 2.1 & -619.245632 & -619.160801 & 2.2 & 2.1 & -619.205051 & -619.121380 \\
\hline 2.0 & 2.2 & -619.245935 & -619.161007 & 2.2 & 2.2 & -619.197332 & -619.114786 \\
\hline 2.0 & 2.3 & -619.247404 & -619.162102 & 2.2 & 2.3 & -619.199662 & -619.110129 \\
\hline 2.0 & 2.4 & -619.249511 & -619.163703 & 2.2 & 2.4 & -619.212928 & -619.121432 \\
\hline 2.0 & 2.5 & -619.251811 & -619.165485 & 2.2 & 2.5 & -619.225074 & -619.133232 \\
\hline 2.0 & 2.6 & -619.254036 & -619.167269 & 2.2 & 2.6 & -619.235103 & -619.143019 \\
\hline 2.0 & 2.7 & -619.256005 & -619.168907 & 2.2 & 2.7 & -619.243344 & -619.151164 \\
\hline 2.0 & 2.8 & -619.257610 & -619.170270 & 2.2 & 2.8 & -619.250133 & -619.157888 \\
\hline 2.0 & 2.9 & -619.258842 & -619.171343 & 2.2 & 2.9 & -619.255754 & -619.163499 \\
\hline 2.0 & 3.0 & -619.259736 & -619.172153 & 2.2 & 3.0 & -619.260416 & -619.168157 \\
\hline 2.0 & 3.1 & -619.260346 & -619.172751 & 2.2 & 3.1 & -619.264131 & -619.171878 \\
\hline
\end{tabular}




\begin{tabular}{|c|c|c|c|c|c|c|c|}
\hline 2.0 & 3.2 & -619.260719 & -619.173149 & 2.2 & 3.2 & -619.267022 & -619.174823 \\
\hline 2.0 & 3.3 & -619.260883 & -619.173367 & 2.2 & 3.3 & -619.269099 & -619.176999 \\
\hline 2.0 & 3.4 & -619.260867 & -619.173436 & 2.2 & 3.4 & -619.270452 & -619.178469 \\
\hline 2.2 & 1.6 & -619.237755 & -619.151967 & 2.4 & 1.6 & -619.220855 & -619.133198 \\
\hline 2.2 & 1.7 & -619.238355 & -619.153136 & 2.4 & 1.7 & -619.216720 & -619.129636 \\
\hline 2.2 & 1.8 & -619.237283 & -619.152529 & 2.4 & 1.8 & -619.209411 & -619.123003 \\
\hline 2 & 1.9 & -619.236562 & -619.151972 & 2.4 & 1.9 & -619.200880 & -619.115196 \\
\hline 2 & 2.0 & -619.237356 & -619.152445 & 2.4 & 2.0 & -619.192467 & -619.107491 \\
\hline 2.2 & 2.1 & -619.239911 & -619.154192 & 2.4 & 2.1 & -619.192140 & -619.101652 \\
\hline 2.2 & 2.2 & -619.243753 & -619.156938 & 2.4 & 2.2 & -619.205816 & -619.112771 \\
\hline 2.2 & 2.3 & -619.248190 & -619.160233 & 2.4 & 2.3 & -619.218787 & -619.125791 \\
\hline 2.2 & 2.4 & -619.252629 & -619.163642 & 2.4 & 2.4 & -619.230018 & -619.135916 \\
\hline 2.2 & 2.5 & -619.256690 & -619.166839 & 2.4 & 2.5 & -619.239471 & -619.144668 \\
\hline 2.2 & 2.6 & -619.260219 & -619.169699 & 2.4 & 2.6 & -619.247773 & -619.152909 \\
\hline 2.2 & 2.7 & -619.263176 & -619.172187 & 2.4 & 2.7 & -619.254832 & -619.159824 \\
\hline 2.2 & 2.8 & -619.265540 & -619.174211 & 2.4 & 2.8 & -619.260595 & -619.165525 \\
\hline 2.2 & 2.9 & -619.267373 & -619.175796 & 2.4 & 2.9 & -619.265344 & -619.170306 \\
\hline 2.2 & 3.0 & -619.268744 & -619.177008 & 2.4 & 3.0 & -619.269299 & -619.174290 \\
\hline 2.2 & 3.1 & -619.269742 & -619.177929 & 2.4 & 3.1 & -619.272649 & -619.177665 \\
\hline 2.2 & 3.2 & -619.270440 & -619.178613 & 2.4 & 3.2 & -619.275371 & -619.180415 \\
\hline 2.2 & 3.3 & -619.270908 & -619.179094 & 2.4 & 3.3 & -619.277523 & -619.182619 \\
\hline 2.2 & 3.4 & -619.271178 & -619.179406 & 2.4 & 3.4 & -619.279099 & -619.184267 \\
\hline 2.4 & 1.6 & -619.221813 & -619.137970 & 2.6 & 1.6 & -619.211884 & -619.119434 \\
\hline 2.4 & 1.7 & -619.224971 & -619.141263 & 2.6 & 1.7 & -619.207825 & -619.115945 \\
\hline 2.4 & 1.8 & -619.226990 & -619.143070 & 2.6 & 1.8 & -619.200787 & -619.109596 \\
\hline 2.4 & 1.9 & -619.229864 & -619.145171 & 2.6 & 1.9 & -619.192959 & -619.102535 \\
\hline 2.4 & 2.0 & -619.234344 & -619.148325 & 2.6 & 2.0 & -619.199569 & -619.109449 \\
\hline 2.4 & 2.1 & -619.240143 & -619.152506 & 2.6 & 2.1 & -619.211859 & -619.119604 \\
\hline 2.4 & 2.2 & -619.246548 & -619.157291 & 2.6 & 2.2 & -619.223702 & -619.129882 \\
\hline 2.4 & 2.3 & -619.252870 & -619.162185 & 2.6 & 2.3 & -619.234427 & -619.139514 \\
\hline 2.4 & 2.4 & -619.258675 & -619.166827 & 2.6 & 2.4 & -619.243765 & -619.148113 \\
\hline 2.4 & 2.5 & -619.263715 & -619.170959 & 2.6 & 2.5 & -619.251665 & -619.155563 \\
\hline 2.4 & 2.6 & -619.267915 & -619.174471 & 2.6 & 2.6 & -619.258335 & -619.162006 \\
\hline 2.4 & 2.7 & -619.271326 & -619.177393 & 2.6 & 2.7 & -619.263896 & -619.167530 \\
\hline 2.4 & 2.8 & -619.274030 & -619.179773 & 2.6 & 2.8 & -619.268915 & -619.172417 \\
\hline 2.4 & 2.9 & -619.276116 & -619.181631 & 2.6 & 2.9 & -619.273080 & -619.176480 \\
\hline 2.4 & 3.0 & -619.277689 & -619.183055 & 2.6 & 3.0 & -619.276402 & -619.179871 \\
\hline 2.4 & 3.1 & -619.278854 & -619.184143 & 2.6 & 3.1 & -619.279116 & -619.182640 \\
\hline 2.4 & 3.2 & -619.279699 & -619.184970 & 2.6 & 3.2 & -619.281436 & -619.185000 \\
\hline 2.4 & 3.3 & -619.280291 & -619.185586 & 2.6 & 3.3 & -619.283413 & -619.185619 \\
\hline 2.4 & 3.4 & -619.280695 & -619.186027 & 2.6 & 3.4 & -619.285051 & -619.188663 \\
\hline 2.6 & 1.6 & -619.211533 & -619.128627 & 2.8 & 1.6 & -619.209719 & -619.115179 \\
\hline 2.6 & 1.7 & -619.216862 & -619.133890 & 2.8 & 1.7 & -619.205780 & -619.111837 \\
\hline 2.6 & 1.8 & -619.221802 & -619.137867 & 2.8 & 1.8 & -619.199060 & -619.109209 \\
\hline 2.6 & 1.9 & -619.227637 & -619.142171 & 2.8 & 1.9 & -619.202942 & -619.112255 \\
\hline 2.6 & 2.0 & -619.234683 & -619.147316 & 2.8 & 2.0 & -619.212526 & -619.121859 \\
\hline 2.6 & 2.1 & -619.242484 & -619.153160 & 2.8 & 2.1 & -619.224521 & -619.131874 \\
\hline 2.6 & 2.2 & -619.250351 & -619.159255 & 2.8 & 2.2 & -619.235636 & -619.141466 \\
\hline 2.6 & 2.3 & -619.257708 & -619.165137 & 2.8 & 2.3 & -619.245456 & -619.150163 \\
\hline
\end{tabular}




\begin{tabular}{|c|c|c|c|c|c|c|c|}
\hline 2.6 & 2.4 & -619.264210 & -619.170476 & 2.8 & 2.4 & -619.253817 & -619.157733 \\
\hline 2.6 & 2.5 & -619.269738 & -619.175128 & 2.8 & 2.5 & -619.260810 & -619.164206 \\
\hline 2.6 & 2.6 & -619.274277 & -619.179030 & 2.8 & 2.6 & -619.266554 & -619.169614 \\
\hline 2.6 & 2.7 & -619.277888 & -619.182189 & 2.8 & 2.7 & -619.271195 & -619.174109 \\
\hline 2.6 & 2.8 & -619.280695 & -619.184696 & 2.8 & 2.8 & -619.274942 & -619.177891 \\
\hline 2.6 & 2.9 & -619.282833 & -619.186647 & 2.8 & 2.9 & -619.278640 & -619.181239 \\
\hline 2.6 & 3.0 & -619.284432 & -619.188129 & 2.8 & 3.0 & -619.281713 & -619.184233 \\
\hline 2.6 & 3.1 & -619.285608 & -619.189245 & 2.8 & 3.1 & -619.284110 & -619.186692 \\
\hline 2.6 & 3.2 & -619.286473 & -619.190104 & 2.8 & 3.2 & -619.285985 & -619.188688 \\
\hline 2.6 & 3.3 & -619.287088 & -619.190763 & 2.8 & 3.3 & -619.287466 & -619.190291 \\
\hline 2.6 & 3.4 & -619.287515 & -619.191248 & 2.8 & 3.4 & -619.288746 & -619.191620 \\
\hline 2.8 & 1.6 & -619.208036 & -619.122604 & & & & \\
\hline 2.8 & 1.7 & -619.212791 & -619.129349 & & & & \\
\hline 2.8 & 1.8 & -619.219181 & -619.134914 & & & & \\
\hline 2.8 & 1.9 & -619.226961 & -619.140755 & & & & \\
\hline 2.8 & 2.0 & -619.235593 & -619.147244 & & & & \\
\hline 2.8 & 2.1 & -619.244567 & -619.154156 & & & & \\
\hline 2.8 & 2.2 & -619.253268 & -619.161060 & & & & \\
\hline 2.8 & 2.3 & -619.261210 & -619.167543 & & & & \\
\hline 2.8 & 2.4 & -619.268120 & -619.173321 & & & & \\
\hline 2.8 & 2.5 & -619.273909 & -619.178265 & & & & \\
\hline 2.8 & 2.6 & -619.278600 & -619.182340 & & & & \\
\hline 2.8 & 2.7 & -619.282311 & -619.185630 & & & & \\
\hline 2.8 & 2.8 & -619.285179 & -619.188219 & & & & \\
\hline 2.8 & 2.9 & -619.287325 & -619.190197 & & & & \\
\hline 2.8 & 3.0 & -619.288903 & -619.191691 & & & & \\
\hline 2.8 & 3.1 & -619.290050 & -619.192810 & & & & \\
\hline 2.8 & 3.2 & -619.290873 & -619.193652 & & & & \\
\hline 2.8 & 3.3 & -619.291462 & -619.194292 & & & & \\
\hline 2.8 & 3.4 & -619.291864 & -619.194773 & & & & \\
\hline 3.0 & 1.6 & -619.206993 & -619.118700 & & & & \\
\hline 3.0 & 1.7 & -619.211179 & -619.126196 & & & & \\
\hline 3.0 & 1.8 & -619.217329 & -619.132815 & & & & \\
\hline 3.0 & 1.9 & -619.226288 & -619.132815 & & & & \\
\hline 3.0 & 2.0 & -619.235875 & -619.139637 & & & & \\
\hline 3.0 & 2.1 & -619.245564 & -619.146976 & & & & \\
\hline 3.0 & 2.2 & -619.254770 & -619.154566 & & & & \\
\hline 3.0 & 2.3 & -619.263070 & -619.161979 & & & & \\
\hline 3.0 & 2.4 & -619.270243 & -619.168836 & & & & \\
\hline 3.0 & 2.5 & -619.276247 & -619.174895 & & & & \\
\hline 3.0 & 2.6 & -619.281115 & -619.180061 & & & & \\
\hline 3.0 & 2.7 & -619.284926 & -619.184342 & & & & \\
\hline 3.0 & 2.8 & -619.287836 & -619.187741 & & & & \\
\hline 3.0 & 2.9 & -619.290013 & -619.190380 & & & & \\
\hline 3.0 & 3.0 & -619.291608 & -619.192403 & & & & \\
\hline 3.0 & 3.1 & -619.292750 & -619.193926 & & & & \\
\hline 3.0 & 3.2 & -619.293552 & -619.195060 & & & & \\
\hline 3.0 & 3.3 & -619.294097 & -619.195903 & & & & \\
\hline 3.0 & 3.4 & -619.294459 & -619.196522 & & & & \\
\hline
\end{tabular}

